\title{
WestVirginiaUniversity
}

THE RESEARCH REPOSITORY @ WVU

Graduate Theses, Dissertations, and Problem Reports

2003

\section{Investigation of ship wakes using LES with various SGS models}

Zeynep N. Cehreli

West Virginia University

Follow this and additional works at: https://researchrepository.wvu.edu/etd

\section{Recommended Citation}

Cehreli, Zeynep N., "Investigation of ship wakes using LES with various SGS models" (2003). Graduate Theses, Dissertations, and Problem Reports. 2530.

https://researchrepository.wvu.edu/etd/2530

This Dissertation is protected by copyright and/or related rights. It has been brought to you by the The Research Repository @ WVU with permission from the rights-holder(s). You are free to use this Dissertation in any way that is permitted by the copyright and related rights legislation that applies to your use. For other uses you must obtain permission from the rights-holder(s) directly, unless additional rights are indicated by a Creative Commons license in the record and/ or on the work itself. This Dissertation has been accepted for inclusion in WVU Graduate Theses, Dissertations, and Problem Reports collection by an authorized administrator of The Research Repository @ WVU.

For more information, please contact researchrepository@mail.wvu.edu. 


\title{
INVESTIGATION OF SHIP WAKES USING LES WITH \\ VARIOUS SGS MODELS
}

\author{
Zeynep N. Cehreli
}

\author{
Dissertation submitted to the \\ College of Engineering and Mineral Resources \\ at West Virginia University \\ in partial fulfillment of the requirements \\ for the degree of
}

\author{
Doctor of Philosophy \\ in \\ Mechanical Engineering
}

Ismail Celik, Ph.D., Chair

John Kuhlman, Ph.D.

Wade Huebsch, Ph.D.

Andrei Smirnov, Ph.D.

Peter A. Chang, Ph.D.

Eric G. Paterson, Ph.D.

Mechanical and Aerospace Engineering Department

\author{
Morgantown, West Virginia \\ 2004
}

Keywords: Large eddy simulation, Subgrid scale models, Ship wakes Copyright 2004 Zeynep N. Cehreli 


\section{ABSTRACT \\ Investigation of Ship Wakes using LES \\ with various SGS Models}

Zeynep N. Cehreli

Turbulence plays an important role in ship wake flows. Without the knowledge of turbulent intensities and length scale information it is virtually impossible to model and predict turbulence characteristics in complex flows, such as ship wake flows. Moreover, the behavior of ship wakes requires an understanding of the turbulence near a free surface. The present study will focus on identifying the contributions of various mechanisms, but primarily that of the free surface, to the process of turbulence generation and dissipation in the wake of a turning ship using the large eddy simulation (LES) technique. LES is applied in conjunction with a random flow generation (RFG) technique originally developed at West Virginia University to provide unsteady inflow boundary conditions. Some refinements are made to extend the capabilities of a readily available LES code in predicting turbulence, including among others, appropriate sub-grid scale (SGS) turbulence models. A modified zero equation SGS model is developed, to account for the free surface effects. Furthermore, a non-linear one equation model is developed and tested to represent the anisotropy of turbulence observed near a free surface. Finally, using LES the turbulence characteristics in the wake of a turning ship are studied and compared to that of a ship cruising on a straight track. 
I dedicate this study to my dad, Suha Cehreli and my mom, Canan Cehreli, whose devotion, love and continuous support made it possible for me to see the end of this endeavor. 


\section{ACKNOWLEDGEMENTS}

I am extremely grateful to Dr. Ismail Celik, my advisor, for his endless encouragement, his friendship and the valuable guidance that he provided throughout this "research journey". He is an inspiration to me with his endless enthusiasm in research and life. Special thanks are also to Dr. John Kuhlman, Dr. Wade Huebsch, Dr. Andrei Smirnov, Dr. Peter Chang and Dr. Eric Paterson, who were very supportive and have given important perspectives to my research while serving on my graduate committee.

This work has been conducted under a DOD EPSCOR project sponsored by US NAVY Office of Naval Research. I would like to acknowledge the financial support of the program monitor, Dr. Edwin P. Rood /Dr. Patrick Purtell.

I would like to thank Dr Street of Stanford University for providing the original LES code. I am grateful to Dr. Mark Hyman of Naval Surface Warfare Center for providing his valuable data for my research.

I would like to express my deepest thanks to Dr. Ibrahim Yavuz, for his endless

friendship and his valuable advices which inspired me throughout my thesis. And also my thanks are given to; Andrew Burt, Raju Pakalapati, Jun Li, Cem Ersahin, Gusheng Hu, 
Shaoping Shi, Sherif Kandil and to the rest of the CFD Group Members, which I am very honored to be a part of.

I would also like to thank to my other friends, especially to Ege Semercioglu and Azadeh Tehranian for their friendship and endless support throughout this journey.

Finally, I would like to thank to my supportive, devoted parents and my dearest brother, Ali Cehreli. Without you, I would not have been able to see the end of this endeavor. 


\title{
Nomenclature
}

\author{
$\mathrm{a}_{\mathrm{ij}} \quad$ A transfer coefficient tensor \\ $\mathrm{a}_{\mathrm{k}} \quad \mathrm{a}$ coefficient to be determined in LES_IQ formulation \\ $\mathrm{B}_{\mathrm{i}} \quad$ Discrete operator for the pressure gradient \\ $\mathrm{C}_{\mathrm{i}} \quad$ the convective terms in the momentum equation, the scales of turbulent \\ fluctuations along each axis \\ $\mathrm{C}_{\mathrm{r}} \quad$ Scale similarity coefficient \\ $\mathrm{C}_{\mathrm{s}} \quad$ Smagorinksy constant \\ $\mathrm{C}_{v 1, . .6}$ Model constants in the nonlinear one equation model \\ D the length of one side of the square \\ $D_{E}$ and $D_{I}$ discrete operators for the explicitly treated off diagonal and the implicitly \\ treated diagonal viscous terms \\ $\mathrm{E}_{\rho} \quad$ the convective terms in the scalar equation \\ f A generic variable for filtering, function in nonlinear one equation model, \\ frequency \\ $F_{E}$ and $F_{I} \quad$ discrete operators for the explicitly treated off diagonal and the implicitly \\ treated diagonal diffusive terms \\ Fij flux in the momentum \\ $\mathrm{F}_{\mathrm{r}} \quad$ Froude number \\ G Filter function \\ $\mathrm{G}^{\mathrm{mn}} \quad$ Mesh skewness tensor \\ h Channel depth, mesh size \\ J Jacobian \\ $\mathrm{J}^{-1} \quad$ Inverse Jacobian \\ $k \quad$ Turbulent kinetic energy \\ $\mathrm{k}^{\text {eff_sgs }} \quad$ the effective sgs kinetic energy \\ $k^{\text {res }} \quad$ the resolved turbulent kinetic energy \\ $k^{\text {tot }} \quad$ The total turbulent kinetic energy
}




\begin{tabular}{|c|c|}
\hline$k_{s g s}$ & SGS turbulent kinetic energy on the test filter \\
\hline$I$ & the identity matrix \\
\hline$l$ & the turbulent characteristic length scale \\
\hline $\mathrm{L}$ & Ship length \\
\hline$L_{i j}^{m}$ & Modified Leonard term of the test scale \\
\hline $\mathrm{N}_{1,2,3}$ & Coefficients in the nonlinear one equation model \\
\hline $\mathrm{N}(\mathrm{M}, \sigma)$ & a normal distribution with mean $\mathrm{M}$ and standard deviation $\sigma$. \\
\hline $\mathrm{P}$ & Total pressure \\
\hline $\mathrm{p}$ & Reduced dynamic pressure, formal accuracy of the numerical scheme \\
\hline $\mathrm{r}_{\mathrm{ij}}$ & Anisotropic velocity correlation tensor \\
\hline $\mathrm{r}$ & the distance to the rotation axis \\
\hline $\mathrm{R}$ & Radius of the curvature \\
\hline $\mathrm{R}_{\mathrm{e}}$ & Reynolds number \\
\hline $\mathrm{Re}_{\mathrm{t}}$ & Turbulent Reynolds number \\
\hline $\mathrm{R}_{\mathrm{j}}$ & Flux in the scalar equation \\
\hline $\mathrm{s}$ & Streamwise distance from IDP, in terms of $\mathrm{L}$ \\
\hline $\mathrm{S}_{\mathrm{i}}$ & Source term of the momentum equation and scalar equation \\
\hline $\bar{S}_{i j}$ & Resolved (large scale) strain rate tensor \\
\hline $\bar{S}_{12}$ & the resolved strain rate at a location near the free surface \\
\hline $\bar{S}_{i}^{\prime}(\phi)$ & the transformation of $\bar{S}_{i}(\phi)$ to the computational domain \\
\hline $\mathrm{t}$ & time \\
\hline $\bar{U}_{m}$ & Contravariant volume flux \\
\hline $\mathrm{u}_{\mathrm{i}},(\mathrm{u}, \mathrm{v}, \mathrm{w})$ & Cartesian velocities in $\mathrm{x}, \mathrm{y}$ and $\mathrm{z}$ direction \\
\hline $\mathrm{u}^{\prime}, \mathrm{v}^{\prime}, \mathrm{w}^{\prime}$ & turbulent intensities in $\mathrm{x}, \mathrm{y}$ and $\mathrm{z}$ direction \\
\hline $\mathrm{u}_{\mathrm{i}}{ }^{*}$ & the intermediate velocity \\
\hline $\mathrm{U}_{\mathrm{o}}$ & the inflow velocity or ship velocity \\
\hline $\mathrm{u}_{\tau}$ & Friction velocity \\
\hline $\mathrm{v}_{\mathrm{i}}$ & time-dependent velocity fluctuations \\
\hline
\end{tabular}




$\begin{array}{ll}\bar{W}_{i j} & \text { Vorticity tensor } \\ \mathrm{w}_{\mathrm{i}} & \text { time-dependent velocity fluctuations } \\ \mathrm{x}_{\mathrm{i}},(\mathrm{x}, \mathrm{y}, \mathrm{z}) & \begin{array}{l}\text { Cartesian coordinates } \\ \mathrm{x}\end{array} \\ \mathrm{x}^{\prime} & \text { Axial distance from the ship } \\ \mathrm{y} & \text { Axial distance from } \mathrm{x}=0\left(\mathrm{x}^{\prime}=0.5 \mathrm{~L}+\mathrm{s}\right) \\ \mathrm{y}_{\mathrm{s}} & \text { Distance from the wall } \\ \mathrm{z}^{*} & \text { the distance from the free surface }\end{array}$




\title{
Greek Nomenclature
}

\author{
$\Delta \quad$ Characteristic length scale \\ $\bar{\Delta} \quad$ Length scale of the grid filter \\ $\Delta^{*} \quad$ A new grid filter width \\ $\Delta x_{1}, \Delta x_{2}, \Delta x_{3}$ Grid spacing in the physical space \\ $\delta \quad$ Channel height \\ $\delta_{\mathrm{ij}} \quad$ Kronecker delta \\ $\varepsilon_{\mathrm{ijk}} \quad$ the permutation tensor \\ $\varepsilon \quad$ Rate of dissipation of turbulent kinetic energy \\ $\xi_{\mathrm{m}} \quad$ Curvilinear coordinates \\ $\kappa \quad$ Von Karman constant \\ $k_{j}^{n} \quad$ a sample of $\mathrm{n}$ wave number vectors of the modeled turbulence spectrum \\ $\tau \quad$ Time scale \\ $\tau_{\mathrm{w}} \quad$ SGS stress term at the wall \\ $\tau_{\mathrm{ij}} \quad$ SGS stress or SGS flux \\ $\alpha \quad$ A material coefficient, grid refinement (coarsening) parameter \\ $\alpha_{\mathrm{T}} \quad$ Turbulence diffusivity in scalar equation \\ $\mu \quad$ dynamic viscosity \\ $\checkmark \quad$ kinematic viscosity \\ $v_{\mathrm{T}} \quad$ Turbulent eddy viscosity \\ $\pi \quad$ pi, $3.141592 \ldots$ \\ $\theta \quad$ Angle of cross section plane normal to the flow to the IDP \\ $\phi \quad$ A scalar \\ $\rho_{0} \quad$ Reference density \\ $\omega_{\mathrm{n}} \quad$ a sample of $\mathrm{n}$ frequencies of the modeled turbulence spectrum \\ $\omega_{\mathrm{y}} \quad$ Vertical vorticity in the turning ship wake simulation
}


$(\nabla \bar{u})_{k l} \quad$ the deformation rate tensor

$\Omega_{\mathrm{s}} \quad$ Angular velocity of the system rotation due to the turning of the ship

$\chi_{j} \quad$ The effect of the SGS motion 


\section{Abbreviations}

$\begin{array}{ll}\text { CD } & \text { Central differencing } \\ \text { CFD } & \text { Computational fluid dynamics } \\ \text { DNS } & \text { Direct numerical simulation } \\ \text { LES } & \text { Large Eddy Simulation } \\ \text { LES_IQ } & \text { Large Eddy Simulation Index of Quality } \\ \text { QUICK } & \text { Quadratic Upstream Interpolation for Convective Kinematics } \\ \text { RANS } & \text { Reynolds Averaged Navier Stokes } \\ \text { RFG } & \text { Random flow generation } \\ \text { SGS } & \text { Subgrid scale }\end{array}$




\section{TABLE OF CONTENTS}

ABSTRACT $\quad$......................................................................................................................... ii

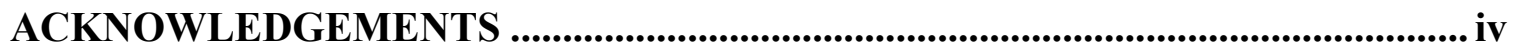

NOMENCLATURE.................................................................................................. vi

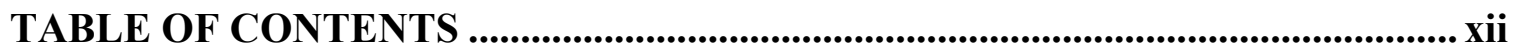

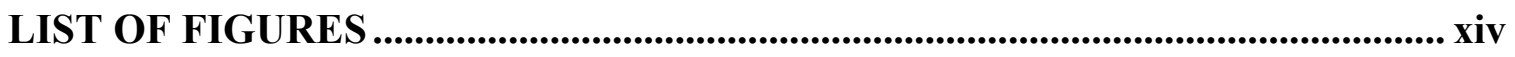

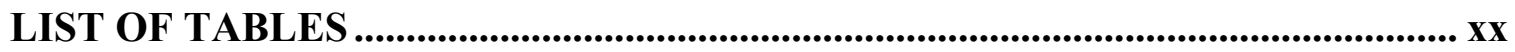

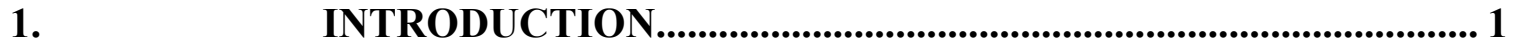

Motivation and Objectives ......................................................... 1

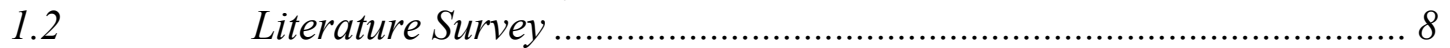

1.2.1 Wake Flow ..................................................................... 8

1.2.2 Free Surface Flows ...................................................... 13

1.2.3 SGS Studies .................................................................. 18

Contributions from the current work ............................................... 23

2. COMPUTATIONAL APPROACH .............................................. 25

$2.1 \quad$ Governing Equations and Navier Stokes solver ............................. 26

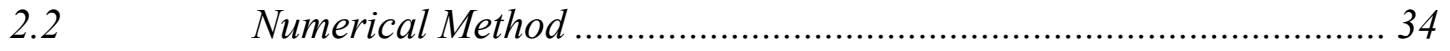

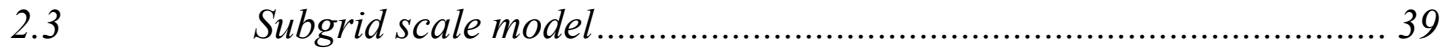

$2.4 \quad$ Random flow generation $(R F G)$ methodology ................................ 47

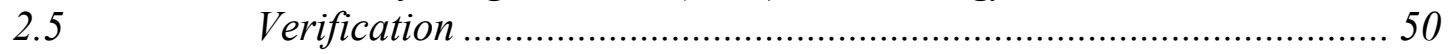

3.

APPLICATION OF EDDY VISCOSITY SGS MODELS .......... 52

3.1 Application of Smagorinsky model to the wake of a Turning Ship..... 53

3.1.1 Conditions and Grids........................................................ 54

3.1.2 Boundary Conditions .......................................................... 58

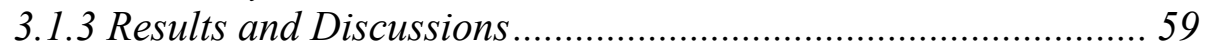


3.1.4 Properties of turbulent ship wakes .................................. 72

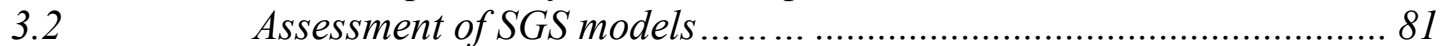

3.2.1 Application of FSM to Ship Wake ........................................ 100

Uncertainty in the present LES ................................................... 122

4.

CONCLUSIONS AND RECOMMENDATIONS........................ 130

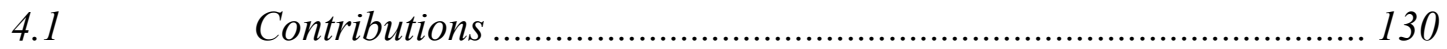

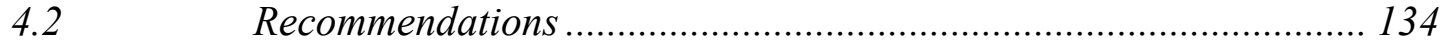

A.

ENERGY SPECTRA .................................................................... 136

B.

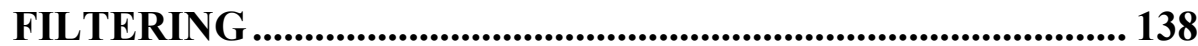

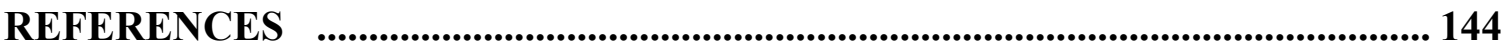




\section{LIST OF FIGURES}

Figure 1.1. Schematic sketch of a ship wake (Hennings et al. 1999) ............................. 9

Figure 1.2. Geometry of the computational region: a) coordinate system b) mesh

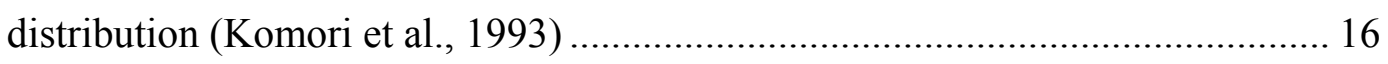

Figure 1.3. Turbulence intensity distributions: a) comparisons between predictions and measurement by LDV (Komori et al., 1990) b) comparisons between the predictions by Komori et al. (1993)'s DNS and those of Kim et al.(1987) and Lam

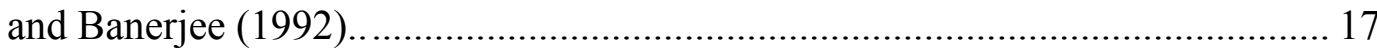

Figure 1.4. Geometry of the computational region having moving free surface

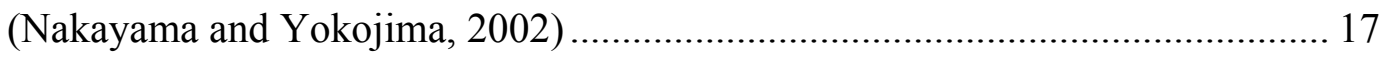

Figure 1.5. Turbulence intensity distributions: a) $\mathrm{Fr}=0.3$ b) $\mathrm{Fr}=0.6$ (Nakayama and Yokojima, 2002).

Figure 3.1. Arleigh-Burke class destroyer (DDG51). 56

Figure 3.2. Turning ship wake: a) The geometry b) velocity profile specified at the IDP (top view) c) The coordinate space system and numerical grid (Only the core region is shown, distances are non-dimensionalized with ship length, L).....

Figure 3.3. Typical instantaneous vertical velocity contours w Coriolis force on different $\mathrm{y}-\mathrm{z}$ plane in the turning ship wake (coarse grid) 61

Figure 3.4. Radial velocity profiles on different y-z planes in the turning ship wake (fine grid) 61 
Figure 3.5. Temporal history of streamwise velocity components at $10^{\circ}$ with the $\mathrm{z}$ axis, and $\mathrm{x}=1.02, \mathrm{y}=-0.002$ (coarse grid), a) w Coriolis force b) w/o Coriolis force 62

Figure 3.6. Temporal history of streamwise velocity components at $10^{\circ} \mathrm{with}$ the $\mathrm{z}$ axis, and $\mathrm{x}=1.02, \mathrm{y}=-0.002$ (medium grid), $\mathrm{Cs}=0.042$. 62

Figure 3.7. Temporal history of streamwise velocity components at $10^{\circ}$ with the $\mathrm{z}$ axis, and $\mathrm{x}=1.02, \mathrm{y}=-0.002$ (medium grid), $\mathrm{Cs}=0.065$

Figure 3.8. The velocity vectors on a vertical plane at an angle of $5^{\circ}$ with the $z$-axis with Coriolis force (coarse grid) 63

Figure 3.9. The velocity vectors on a vertical plane at an angle of $5^{\circ}$ with the $\mathrm{z}$-axis without Coriolis force (coarse grid)

Figure 3.10 . The velocity vectors on a vertical plane at an angle of $5^{\circ}$ with the $z$-axis (medium grid) a) $\mathrm{Cs}=0.042 \mathrm{~b}$ ) $\mathrm{Cs}=0.065$

Figure 3.11. The velocity vectors on a vertical plane at an angle of $25^{\circ}$ with the z-axis with Coriolis force (coarse grid) ..... 66

Figure 3.12 . The velocity vectors on a vertical plane at an angle of $25^{\circ}$ with the $\mathrm{z}$-axis without Coriolis force (coarse grid)

Figure 3.13 . The velocity vectors on a vertical plane at an angle of $25^{\circ}$ with the $\mathrm{z}$-axis (medium grid) a) $\mathrm{Cs}=0.042 \mathrm{~b}$ ) $\mathrm{Cs}=0.065$....

Figure 3.14. The comparison of the resolved turbulence kinetic energy for ship cruising on a circular track. 68

Figure 3.15. Velocity vectors at $\mathrm{x} / \mathrm{L}=0.2$ : a) Non-turning ship (Shi et al. 2001) (fine grid)

b) Turning ship (fine grid). 70

Figure 3.16. Velocity vectors at $\mathrm{x} / \mathrm{L}=0.65$ a) Non-turning ship (Shi et al. 2001) (finest grid) b) Turning ship (finest grid) 71

Figure 3.17. Velocity vectors at $\mathrm{x} / \mathrm{L}=0.65$ for turning ship wake a) Case- $1 ; \mathrm{Re}=1.5 \times 10^{9}$ :

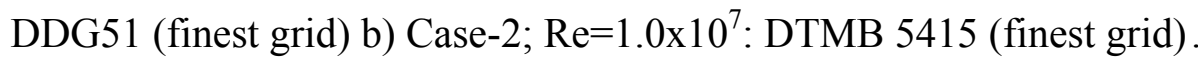
71

Figure 3.18 . Temporal history of streamwise velocity components at $10^{\circ}(x=1.02, y=-$ 0.001 and $\mathrm{z}=-3.18$ ) (finest grid) and $\mathrm{Cs}=0.052 \mathrm{a}$ ) Case-1 b) Case-2 74

Figure 3.19 a) The mean axial velocity contour at the inlet data plane (IDP) for nonturning ship (Shi et al. 2001) (finest grid) b) The mean axial velocity contours at the IDP for turning ship wake (finest grid) c) The mean axial velocity contours after 
subtracting solid body rotation contribution at the IDP for turning ship wake (finest grid) 76

Figure 3.20. a) The axial flow field provided by RFG at the inlet plane (IDP) for turning ship wake b) Enlarged view of A (finest grid) ..... 77

Figure 3.21. a) Axial velocity contour at $x / L=0.65$ for non-turning ship (Shi et al. 2001) (finest grid) b) Axial velocity contours subtracting solid body rotation contribution at $\mathrm{x}^{\prime} / \mathrm{L}=0.65$ for turning ship wake (finest grid) 78

Figure 3.22. a) Predicted velocity contours at $\mathrm{x} / \mathrm{L}=1.20$ for non-turning ship-(Shi et al. 2001) (finest grid) b) Macro wake measurements at $\mathrm{x} / \mathrm{L}=1.20$ by Hoekstra $\&$ Ligtelijn (1991) for ship model No. $5452\left(\operatorname{Re} \sim 1 \times 10^{7}\right)$ 79

Figure 3.23. Velocity vectors for turning ship using standard Smagorinsky model (finest grid) on a vertical plane at an angle of a) $5^{\circ}\left(\mathrm{x}^{\prime} / \mathrm{L}=0.8\right)$ b) $10^{\circ}\left(\mathrm{x}^{\prime} / \mathrm{L}=1.02\right)$ 82

Figure 3.23. Velocity vectors for turning ship using standard Smagorinsky model (finest grid) on a vertical plane at an angle of c) $20^{\circ}\left(x^{\prime} / L=1.55\right)$ d) $30^{\circ}\left(x^{\prime} / L=1.75\right)$ 83

Figure 3.24. Energy spectra of the velocity fluctuations a) Non-turning ship (Shi et al. 2001) (finest grid) b) Turning ship (finest grid) 84

Figure 3.25. Predicted vertical vorticity ( $\omega y)$ for a) Non-turning ship (Shi et al. 2001) (finest grid) b) Turning ship wake (finest grid) (Case 2) ............................................ 85

Figure 3.26. Geometry of the open channel flow case ................................................... 87

Figure 3.27. Resolved turbulence intensities in a) streamwise direction: (values are normalized with $\mathrm{u}_{\tau}$ )

Figure 3.27. Resolved turbulence intensities in b) vertical direction; c) vertical direction enlarged near the free surface region :(values are normalized with $\mathrm{u}_{\tau}$ ) 88

Figure 3.27. Resolved turbulence intensities in d) spanwise direction: (values are normalized with $\mathrm{u}_{\tau}$ ) 89

Figure 3.28. Comparison of the mean velocity profile. 89

Figure 3.29 a) Vertical distributions of turbulence intensities; Comparisons between the present FSM+NWM and measurements, predictions by DNS and LES studies in streamwise direction: (values are normalized with $\mathrm{u}_{\tau}$ ) 92 
Figure 3.29 b\&c) Vertical distributions of turbulence intensities; Comparisons between the present FSM+NWM and measurements, predictions by DNS and LES studies in vertical and spanwise directions respectively: (values are normalized with $\mathrm{u}_{\tau}$ ) ....... 93

Figure 3.30. Vertical distributions of turbulence intensities; Comparisons between the present NOEM+NWM with FSM+NWM and measurements, predictions by DNS and LES studies in a) streamwise b) vertical directions: (values are normalized with

$\left.\mathrm{u}_{\tau}\right)$

Figure 3.30 c) Vertical distributions of turbulence intensities; Comparisons between the present NOEM+NWM with FSM+NWM and measurements, predictions by DNS and LES studies in spanwise directions: (values are normalized with $\mathrm{u}_{\tau}$ ) ............... 97

Figure 3.31. Comparison of the mean flow velocity profile ........................................ 98

Figure 3.32. Temporal history of streamwise velocity components at $10^{\circ}(x=1.02 y=-$ $0.001 \mathrm{z}=-3.154881$ ) (fine grid) a) without b) with FSM 103

Figure 3.33. The mean axial velocity color contours at a) IDP (Hyman, 2001) b) an angle of $5^{\circ}\left(x^{\prime} / L=0.80\right)$ without FSM $\left.b\right)$ an angle of $5^{\circ}\left(x^{\prime} / L=0.80\right)$ with FSM (fine grid) 104

Figure 3.34. The mean axial velocity line contours at a) IDP (Hyman, 2001) b) an angle of $5^{\circ}\left(x^{\prime} / L=0.80\right)$ without FSM $\left.b\right)$ an angle of $5^{\circ}\left(x^{\prime} / L=0.80\right)$ with FSM (fine grid) 105

Figure 3.35. The mean vertical velocity color contours at a) IDP (Hyman, 2001) b) an angle of $5^{\circ}\left(x^{\prime} / L=0.80\right)$ without FSM $\left.b\right)$ an angle of $5^{\circ}\left(x^{\prime} / L=0.80\right)$ with FSM (fine grid) 106

Figure 3.36. The mean vertical velocity line contours at a) IDP (Hyman, 2001) b) an angle of $5^{\circ}\left(\mathrm{x}^{\prime} / \mathrm{L}=0.80\right)$ without FSM $\left.\mathrm{b}\right)$ an angle of $5^{\circ}\left(\mathrm{x}^{\prime} / \mathrm{L}=0.80\right)$ with FSM (fine grid) 107

Figure 3.37. The mean transverse velocity color contours at a) IDP (Hyman, 2001) b) an angle of $5^{\circ}\left(\mathrm{x}^{\prime} / \mathrm{L}=0.80\right)$ without FSM $\left.\mathrm{b}\right)$ an angle of $5^{\circ}\left(\mathrm{x}^{\prime} / \mathrm{L}=0.80\right)$ with FSM (fine grid) 108 
Figure 3.38. The mean transverse velocity line contours at a) IDP (Hyman, 2001) b) an angle of $5^{\circ}\left(\mathrm{x}^{\prime} / \mathrm{L}=0.80\right)$ without FSM $\left.\mathrm{b}\right)$ an angle of $5^{\circ}\left(\mathrm{x}^{\prime} / \mathrm{L}=0.80\right)$ with FSM (fine grid)

Figure 3.39. The mean velocity profiles at 3 vertical locations at a) IDP (Hyman, 2001) b) an angle of $5^{\circ}\left(x^{\prime} / L=0.80\right)$ without FSM $\left.b\right)$ an angle of $5^{\circ}\left(x^{\prime} / L=0.80\right)$ with FSM (fine grid) 110

Figure 3.40. The mean velocity profiles at 3 vertical locations at a) an angle of $15^{\circ}$ $\left(\mathrm{x}^{\prime} / \mathrm{L}=1.30\right)$ without FSM $\left.b\right)$ an angle of $15^{\circ}\left(\mathrm{x}^{\prime} / \mathrm{L}=1.30\right)$ with FSM (fine grid) ..... 111

Figure 3.41. The mean velocity profiles at 3 spanwise locations at a) IDP (Hyman, 2001)

b) an angle of $5^{\circ}\left(x^{\prime} / L=0.80\right)$ without FSM b) an angle of $5^{\circ}\left(x^{\prime} / L=0.80\right)$ with FSM (fine grid))

Figure 3.42. The mean velocity profiles at 3 spanwise locations at a) an angle of $15^{\circ}$ $\left(\mathrm{x}^{\prime} / \mathrm{L}=1.30\right)$ without FSM $\left.\mathrm{b}\right)$ an angle of $15^{\circ}\left(\mathrm{x}^{\prime} / \mathrm{L}=1.30\right)$ with FSM (fine grid) $) . . .113$

Figure 3.43. The variation root mean square velocities with depth at an angle of $5^{\circ}$ $\left(x^{\prime} / L=0.80\right)$ a) without FSM b) with FSM (fine grid). 114

Figure 3.44. The variation root mean square velocities with depth at an angle of $15^{\circ}$ $\left(x^{\prime} / L=1.30\right)$ a) without FSM b) with FSM (fine grid) ..... 115

Figure 3.45. The velocity vectors on a vertical plane at an angle of $5^{\circ}$ with the z-axis: a) without and b) with FSM (fine grid) 116

Figure 3.46. The velocity vectors on a vertical plane at an angle of $15^{\circ}$ with the z-axis: a) without and $b$ ) with FSM (fine grid)..... 117

Figure 3.47. The velocity vectors on a vertical plane at an angle of $25^{\circ}$ with the z-axis: a) without and $b$ ) with FSM (fine grid)..... 118

Figure 3.48. The predicted vertical vorticity, $\omega \mathrm{y}$, contours at $\mathrm{y}=-0.1 \mathrm{E}-02$ in the ship wake: a) without and b) with FSM (fine grid)

Figure 3.49. The comparison of the resolved turbulence kinetic energy normalized w.r.t its inlet value, $\mathrm{k}_{\text {inlet }}=2.5 \mathrm{e}-02$ (at $\mathrm{y}=-0.1 \mathrm{E}-02$ and $\mathrm{z}=-3.18$ ) (Turning ship), $\mathrm{Re}=1.0 \times 10^{7} \mathrm{k}_{\text {inlet }}=3.3 \mathrm{e}-03$ (at the centerline) (Non-turning ship), $\mathrm{Re}=4.65 \times 10^{6}$ for different grid resolution without FSM 120 
Figure 3.50. The comparison of the resolved turbulence kinetic energy normalized w.r.t its inlet value, $\mathrm{k}_{\text {inlet }}=2.5 \mathrm{e}-02$ (Turning ship), $\mathrm{Re}=1.0 \times 10^{7} \mathrm{w}$ and without FSM (at $\mathrm{y}=$ $-0.1 \mathrm{E}-02$ and $\mathrm{z}=-3.18$ ) (fine grid) .....

Figure 3.51. Resolved turbulence kinetic energy of the wake simulation of a ship cruising on a straight track normalized w.r.t. its inlet value $\left(\mathrm{k}_{\text {inlet }}=3.3 \mathrm{e}-03\right)$

Figure 3.52. Large Eddy Simulation Index of Quality (LES_IQ) for the wake simulation of a ship cruising on a straight track 128

Figure 3.53. Large Eddy Simulation Index of Quality (LES_IQ) for the wake simulation of a ship cruising on a circular track

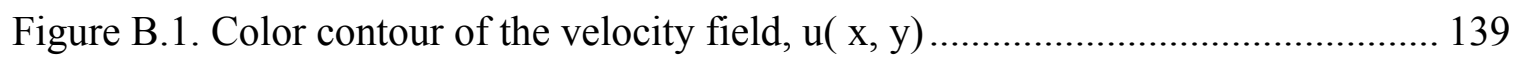

Figure B.2. a) \& b) Small filter length of equal size in both directions........................ 140

Figure B.2. c) \& d) Large filter length of equal size in both directions....................... 141

Figure B.3. a) \& b) Large filter length in the y-direction ........................................... 142

Figure B.4. a) \& b) Large filter length in the $x$-direction ......................................... 143 


\section{LIST OF TABLES}

Table 3.1. Comparison of the maximum values of the root mean square fluctuations for some locations in units of $(\mathrm{m} / \mathrm{s})$ for non-turning ship........................ 80 


\section{Chapter 1}

\section{INTRODUCTION}

\subsection{Motivation and objectives}

The dynamic features of the flow in the wake of surface ships are important with regards to ship design, environment, and especially military applications. Turbulence is the key player in ship wake flows. Information on turning ship wake dynamics is very rare and experiments for ship wakes are very difficult because the ships take a long time to design, they are very expensive, and due to big scale and complexity of the ship wakes, detailed information can not be provided and scaling laws are not realizable. However, with the development of satellite remote sensors such as synthetic aperture radar (SAR) that has been operating from a NASA scientific satellite called SEASET, the images of ships and their wakes can be recorded. This development is important in the area of Naval hydrodynamic research, but the surface wake behind a ship is a region that consists of very complex hydrodynamic flow phenomena that is not well understood (Reed et al., 1990). Moreover, because of its great potential and rapid growth in computer power, 
numerical simulations have been emerging to be a primary tool for prediction of complex turbulent flows such as that observed in surface ship wakes.

Work such as described in this dissertation can be used to improve the fidelity of U.S. Navy threat vulnerability prediction tools applied to surface ships, such as for ARL Penn State Technology Requirements Model (TRM) (Paterson, 2003). Such tools, if applied, for instance, to the acoustic detection of a surface ship via air bubbles entrained in its wake, depend heavily upon knowledge of turbulent, bubbly wake flows.

The physics of turbulent flow are governed by the time dependent, threedimensional Navier-Stokes equations. Although these equations can properly describe turbulent flows, it is very costly, and in many cases not necessary, to obtain exact solutions because of the resolvable, but prohibitively expensive small time and length scales associated with turbulent flows.

Numerous turbulence models are available in the literature that can be used for calculating the Reynolds stresses. These can be classified according to the range of scales they capture in the whole turbulence spectrum. Most of the results of Computational Fluid Dynamics (CFD) applied to the flows around ship hulls are based on ReynoldsAveraged Navier-Stokes (RANS) equations using various turbulence models (Reed et al., 1990; Paterson et al., 1996; Ratcliffe, 1998). RANS is completely statistical and models all turbulent fluctuations. In practical applications, for high Reynolds number flows, RANS simulations are widely used as the RANS equations calculate the averaged flow properties and the computing time required is less compared to other numerical predictions of turbulent flows. It does fairly well for equilibrium flows, such as boundary layers with mild or zero pressure gradients and it does very well for isotropic turbulence. 
However RANS equation modeling can not provide instantaneous information concerning turbulent flows. Flows with massive separation or inherent unsteadiness, vortex shedding behind a cylinder for example are beyond the reach of RANS. Also in RANS, there is a need for introduction of a number of phenomenological closure assumptions and empirical flow dependent constants (Domaradzki et al., 1993). Advances in computer technology and numerical algorithms helped to successfully simulate fairly complex flows in detail using the large eddy simulation (LES) technique (see e.g. Ferziger, 1993; Piomelli, 1998; Sohankar et al., 2000; Hamba, 2001). This technique is designed to simulate the unsteady behavior of large energy containing coherent structures. This technique is preferred to direct numerical simulations (DNS). DNS is too restrictive in that it requires prohibitive computational resources for flow problems of practical engineering applications, i.e. the computational cost of DNS is very high (proportional to Reynolds number to the power of 3 ; $\operatorname{Re}^{3}$, (Piomelli, 1999)) and inapplicable to high Reynolds number flows. However, turbulent motions of all scales (down to the Kolmogorov scales) are resolved. Consequently, DNS can be regarded as an experiment - taken without instrusive measuring techniques. This alone ensures its usefulness as a research tool.

However, it is more desirable to use LES when the details of turbulence structures, at least most energetic turbulent vortices and eddies, are needed to be captured. In LES, the time dependent, three dimensional space filtered Navier Stokes equations are solved using accurate numerical schemes and fine grid resolution. This approach aims at resolving the most important (energetic), large turbulent scales (which are affected by the flow geometry); only the effect of unresolved small scales (which are 
filtered out) need to be empirically modeled. In other words, LES resolves the most of inertial range and models the dissipations scales. Therefore, as in RANS, the need arises to model the effects of the unresolved scales. But similar to DNS, LES produces a threedimensional, time-dependent solution. By only computing explicitly the largest, most energy containing, scales of motion while modeling the small scales LES computations can be substantially less expensive than those of DNS at a given Reynolds number. This makes LES a useful tool for calculating turbulent flows at Reynolds numbers beyond the reach of DNS. Because the large scales are represented explicitly, LES is usually more accurate and reliable than RANS. Moreover, the modeling of the small scales of the motions is simpler than the modeling of all the scales of motions required by RANS and better accuracy can be achieved, i.e. in three-dimensional flows for which most turbulence models (especially two-equation models) are known to be inadequate (Piomelli et al., 1997). Since the relatively small scales are more homogeneous and universal (isotropic), and therefore less affected by the boundary conditions, LES is amenable to more universal models (It is hoped that such modeling in LES can be accomplished with less empiricism and with greater help from the theories of homogenous turbulence (Domaradzki et al., 1993)). According to the theory, the unresolved scales are proportional to the time step and the grid spacing used in the numerical mesh. Hence, to the extent sufficiently fine grid resolution and small time steps are used, the importance of empirical modeling is diminished. In this way, it is possible to directly compute the turbulent fluctuation resulting from relatively larger eddies. Since the flow information at small scales may not be important for engineering applications, LES is becoming an important and powerful tool in studying turbulence (Piomelli, 1999). 
Smagorinsky (1963) \& Lilly (1967) successfully applied LES in meteorological applications and atmospheric boundary layers. Some examples for engineering applications are fully developed turbulent channel flows (Moin and Kim, 1982 ; Piomelli, 1993), turbulent wake flows (Shi et al., 2000), turbulent mixing layers (Vreman et al., 1997) flow past circular cylinders (Sohankar et al., 2000), flow around a surface mounted cube (Krajnovic et al., 1999), and free surface flows (Dommermuth et al., 1993; Dommermuth et al., 1996, Salvetti et al., 1997, Shi et al., 2000).

The behavior of wakes behind ships requires an understanding of the turbulence structure near a free surface. There is very limited information on free surface turbulence in the literature due to difficulties in the measurement of turbulence and velocity near a free surface. The DNS of free surface flows is limited to low Reynolds number flows and simple geometries. In spite of all these, many researchers have made great efforts in the understanding of free surface flows, such as Komori et al. (1982) and Sarpkaya et al. (1994). However, LES provides an effective tool for tackling this type of problem (Salvetti et al., 1997).

This study focuses on the application of the eddy viscosity SGS models to the wake behind a ship cruising on a circular track, with or without the modification of the effect of free surface. However, an alternative SGS model needs to be developed or an existing model needs to be improved to predict turbulence near the free surface. This need arises from the fact that all the models used, assume isotropic SGS turbulence near the free surface. Hence, one of the main objectives of this study is to investigate the behavior of the SGS models in LES of free surface turbulent flows. 
To achieve more accurate ship wake simulations and principally still remain within the realistic computational constraints, the flow past a surface ship is divided into two parts. steady-state RANS calculations around the ship hull, and non-steady LES of the wake. For this reason, LES computations are started from a plane behind the ship. This is accomplished by using the Random flow generation (RFG) technique (Celik et al., 1999, Shi et al., 2000 and Smirnov et al., 2000), which requires as input for a time averaged flow field at the inlet data plane. A considerable amount of work in random flow generation has been performed in the area of particle dispersion modeling using the RANS approach (Zhou and Leschziner, 1991, Zhou and Leschziner, 1996, Li et al., 1994). RANS modeling produces smooth flow fields, which do not accurately disperse particles that are embedded in the flow. To correct this turbulent Reynolds stresses are used to generate temporally and spatially correlated fluctuations, such that the resultant instantaneous velocity can be superimposed on the particles to induce a realistic dispersion. A number of approaches found in the literature (Li et al., 1994, Bechara et al., 1994, Fung et al., 1992) are based on a variant of spectral method of generating an isotropic continuous flow-field proposed earlier by Kraichnan (1970). However, this flow-field does not satisfy the requirement of spatial inhomogeneity and anisotropy of turbulent shear stresses, which may be important in realistic flows. The method of Zhou and Leschziner (1991) complies with the latter requirement, but the resultant flow field does not satisfy the continuity condition and is spatially uncorrelated. For homogeneous isotropic turbulence, the initial conditions can also be constructed as described by Ferziger (1983). The approach is based on a vector curl operation and forward/backward Fourier transforms. The extension of this method to anisotropic inhomogeneous flows is 
not trivial. At least one study presents a successful application of Kraichnan's method to anisotropic flows (Maxey, 1987). The technique is based on filtering and scaling operations applied to the generated isotropic flow-field to filter only the vectors with the prescribed correlations. Again, the filtering operation may be expensive computationally. The method presented in this study is different in that it is based only on scaling and simple coordinate transformation operations.

Reynolds Averaged Navier Stokes (RANS) calculations (Hyman, 2001) are used to provide the RFG procedure with the information needed on the inflow boundary. The further downstream development of the wake flow is calculated via LES. This combined approach enables LES of high Reynolds number flows with complicated geometries. The effect of the ship hull on the flow field is implicitly embedded in the mean flow prescribed at the inflow plane, even though the LES procedure starts at a plane right behind the body (1.5 ship length a stern).

Another goal of this study is to investigate the turbulent flow dynamics in the wake of a turning ship and compare the results to those of a ship cruising on a straight track (see Shi et al., 2002). For the turning ship, the reference frame is noninertial and the effects of the Coriolis and centrifugal forces on the vortical structures are of great importance. Coriolis force is a fictitious force exerted on a body when it moves in a rotating reference frame. It is called a fictitious force because it is a byproduct of measuring coordinates with respect to a rotating coordinate system as opposed to an actual body force. The centrifugal force is the outward directed fictitious force exerted on a body when it moves azimuthally in a noninertial rotating reference frame. In this study, 
the influence of the Coriolis and centrifugal forces on turbulence generation on the flow field will be investigated.

\subsection{Literature Survey}

\subsubsection{Wake Flow}

Wake flows are characterized by rapid streamwise adjustments and are strongly inhomogeneous along all three directions (Shi, 2000). In the literature, there are numerous descriptions of a ship's wake; due to its complexity it is not well understood and ship wake flow dynamics is still an ongoing research area. William Thompson (Lord Kelvin) (1887) was the first to explain the wave pattern generated on the water surface by a moving disturbance. The hydrodynamic phenomenon which occurs in a ship's wake is a result of the generation of turbulence by the ship hull and its peripherals. This wake develops and grows in time and characterizes the scale of turbulence. The properties of the ship wake depend on the size, shape, the speed, and the propulsion system of the ship.

Figure 1.1 illustrates a hydrodynamic wake at sufficiently high speeds. It consists of white water (wake generation region), viscous wake, propeller wake, and Kelvin wave pattern. The white water (local wave disturbance region) originates at the region around and behind the ship up to two or three ship lengths. It is the region where surface foam (entrainment of the air at and below the free surface), bubbles, and strong turbulence is generated and rapidly decayed. The amplitude of these waves depends strongly on the shape, speed and the propulsion system of the ship. This near wake region is also the 
place where these features decay rapidly. The viscous (turbulent) wake extends up to the far field region and the flow is characterized by the wave drag and the drag of the hull. It is this region where large-scale vortical flows occur. A turbulent wake can be several kilometers long and the exact structure of this smooth water region depends on the ship. The turbulence behaves like an additional viscosity that dampens the surface waves (Milgram et al., 1993). The bubbly turbulent wake is one of the main features to be used for detecting ships. In the open literature, there are some limited numerical experiments and full scale measurements of the turbulent wake behind a ship. Swean (1987) solved the steady, parabolic, incompressible time averaged Navier-Stokes equations for the high speed combatant wake, normalized by its beam length and its velocity to obtain the wake profile by using k- $\varepsilon$ turbulence model. Meadows et al. (1994) measured the Lagrangian velocity profiles and compared the experimental and numerical longitudinal surface wake

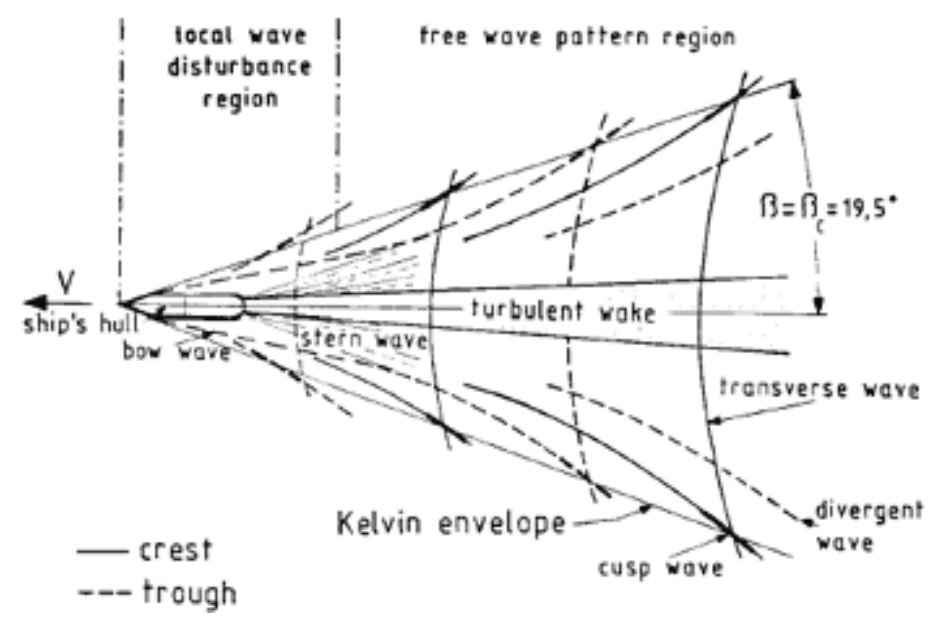

Figure 1.1. Schematic sketch of a ship wake (Hennings et al. 1999) 
velocities, normalized by ship length and velocity. The Kelvin wave pattern (V-shaped) consists of two arms (Kelvin arms). It consists of the superposition of waves, "transverse" and "diverging" wave crests, which propagate in a direction at 145 degrees to the positive x-axis (Hoekstra \& Ligtelijn, 1991). It is a characteristic surface wave pattern resembling a wedge-shaped region behind the ship (Melsheimer et al., 1999). It plays an important role in the development of the wake that is imaged by SARs (Reed et al., 1990). Many researchers have studied Kelvin wakes both experimentally and theoretically, however, the experimental results have mostly been done for idealized mathematical hull forms, that have few characteristics of real ships (Reed et al., 1990). Milgram (1988) as well analyzed the Kelvin wave system hydrodynamically. In the far region, the wake decays relatively slow and steadily, directly proportional to $\mathrm{x}$, being the axial distance from the ship. The wave amplitudes also depend on the ship. Further downstream, the surface foam and bubbles decay and the surface roughness and thermal characteristics gradually return to those of the surrounding ambient surface (Reed et al., 1990). In the far wake of a ship, the effects of turbulent dissipation are minimal (Dommermuth et al., 1993).

The vorticity dynamics of wake flows is a complex phenomenon. The interaction of an underwater jet with a free surface is a simpler flow that has similar characteristics to the wake of a body interacting with the free surface (Reed et. al., 1990). The jet is laminar at the beginning, then vortex rings quickly develop around the jet, because of the interaction of the jet with the free surface, then transition to turbulence starts to develop. In addition, in Madnia's (1989) experimental study on a round jet near a free surface, it is seen that the free surface is not acting like a simple plane of symmetry. Naudascher 
(1965) studied the wake of ship-like bodies. He observed that the wake width had a power law behavior. Buller \& Tunaley (1989)'s measurements have shown that the power law behaviour was $x^{1 / 4}$. This was also indicated by Milgram et al. (1993) who made field measurements for ship wakes. They found that the wake width had a power law behavior of $\mathrm{x}^{1 / 5}$ where $\mathrm{x}$ is the axial distance from the ship. Hoekstra \& Ligtelijn (1991)'s experimental study indicated that the turbulent kinetic energy had an asymptotic behavior of $\mathrm{x}^{-4 / 5}$. Dommermuth et al. (1996) studied free surface flows using LES. They showed the probability distributions of the velocity field in the wake. Comparison of their simulations with the results from the field and laboratory experiments showed good agreement. They predicted the kinetic energy, the attenuation of the mean axial velocity field and the enhanced spreading of the wake near the free surface.

Hoekstra and Aalbers (1997) has done an experimental study on the structure of the wake of unpropelled hulls and found very interesting structures of these "nominal wakes". Without the effect of the propeller, they observed the presence of longitudinal vortices, two pairs of counter rotating vortices, namely bilge vortices (close to the stern in the bilge region), one on either side of the symmetry plane, close to each other and they both tend to move slowly downward. The other pair, called "side vortex pair" generated near the free surface is observed to be far apart. These side vortices tend to move away from the longitudinal symmetry plane of the ship as the distance gets far from the stern. As these vortex pairs drift in the spanwise direction, they get weaker and eventually disappear. In the longitudinal direction, the pattern of the velocity contour plots showed an "upside-down rimmed hat" shape, two side regions close to the free surface and one in the center. Hoekstra and Aaelbers (1997) found that the smallest axial velocity was in the 
wake's symmetry plane near the free surface. Experiments indicate that when strong side vortices are produced, the minimum value occurs below the free surface, but near the cores. Benilov et al.'s (2000) experimental study and the numerical modeling have indicated that they could detect the ship wake turbulence well, and Kolmogorov's inertial range could be identified, even in the far wake region. The production and strength of these vortexes are very much dependent on the ship's shape. Sometimes only one vortex pair can be observed, either bilge or side vortex (Reed et al, 1990). Particularly, the nominal wake is symmetric near the free surface; however, propeller, rudder and combination of bilge vortices usually will produce strong asymmetries.

For wake signatures, turbulence activity near the free surface is of great importance. The convenient measure of the turbulence is the turbulent kinetic energy, which is observed to be insensitive to the ship shape. As stated by Hoekstra and Aalbers (1997), the maximum values of the root mean square fluctuations for some locations in units of $(\mathrm{m} / \mathrm{s})$ are found to be,

$$
\begin{aligned}
& 0.106 \pm 0.02 \text { at } x / L=0.25 \\
& 0.067 \pm 0.01 \text { at } x / L=0.60 \\
& 0.047 \pm 0.01 \text { at } x / L=1.00
\end{aligned}
$$

where $\mathrm{L}$ is the ship length. 


\subsubsection{Free Surface Flows}

As stated before, the behavior of wakes behind ships requires an understanding of the turbulence structure near a free surface. The free surface phenomena are of more importance in many respects than the flow behavior at large depths. Due to difficulties in measurement and simulation, the information about the turbulence structures near the free surface is very limited. The implications of free surface boundary conditions in near surface modeling are quite clear.

Some of the methods used for turbulent free surface simulation in the literature are DNS (Komori et al., 1993; Handler et al., 1993; Dommermuth et al., 1993; Borue et al., 1995; Nakayama et al., 2002) and RANS simulations, applied to steady flows (Celik and Rodi, 1984; Farmer et al., 1994; Stern and Wilson, 2000). LES is perceived as an effective tool for tackling this type of problems and it can capture the coherent turbulence structures near the free surface (Salvetti et al., 1997; Shi et al., 2000). The enhanced spreading of the wake near the free surface can be predicted well by LES (Dommermuth

et al., 1996). There have also been experimental studies to understand the behavior of turbulence near a free surface (Nezu and Rodi, 1985; Nezu and Rodi, 1986; Gonzalez et al., 1996).

Shi et al. (2000) summarized the nature of near surface turbulent structures. When approaching the free surface, the vertical velocity fluctuations are damped and the energy is redistributed to horizontal motions via pressure fluctuations. The surface normal vortices deform the free surface, large eddies with axes nearly parallel to the free surface are flattened by the effect of the free surface and the free surface acts like a highly 
stratified layer. The eddies generated by the bursting motions in the wall region hit the free surface and are lifted up to become the surface renewal eddies (Komori et al., 1993) (note that this mechanism is absent in the wake).

Nezu and Rodi (1986) could not find any difference in open or closed channel measurements for the vertical intensity for the region $\mathrm{y} / \mathrm{h}<0.9$, y being the distance from the free surface and $\mathrm{h}$ the channel depth. It can be stated that, probably, the free surface effects are confined in the region $y / h>0.9$. Moreover, Nezu and Nakagawa (1993) stated that the most essential feature of the free surface is that the vertical fluctuations are damped such that they decrease rapidly as the free surface is approached. This feature was shown experimentally by Smutek (1969), Komori et al. (1982) and phenomenologically by Hunt (1984).

Komori et al. (1987) and Komori et al. (1990) also observed that the streamwise intensity increases slightly in the region $y / h>0.9$, whereas the vertical intensity decreases strongly. This indicates that the redistribution of the turbulent energy among the three components of turbulent intensity do not follow the similarity law due to the free surface's damping effect. Nezu and Nakagawa (1993) stated that the turbulent kinetic energy decreases when the free surface is approached, since the generation is much smaller than its dissipation. The decrease of the kinetic energy has been observed experimentally by Nezu (1977), Komori et al. (1987) and analytically by Rood (1998), and also numerically (DNS) by Handler et al. (1993).

Nezu and Nakagawa (1993) stated that the difference between the closed or open channel flows is that the turbulent eddy viscosity is not zero at the symmetrical axis of the closed channel, whereas it approaches zero at the free surface in open channels. This 
phenomena is explained by Hunt (1984) as the vertical scale of the macro eddies reduces and becomes lower because the macroscale eddy would impinge on the free surface and would be distorted by the free surface, the vertical intensity will also be damped. If one assumes that eddy viscosity is directly proportional with the macroscale eddy size and the vertical intensity, the surface damping effects of the free surface would cause the eddy viscosity to approach zero. Celik and Rodi (1984) pointed out that the eddy viscosity must be reduced near the free surface where the velocity normal to free surface decreases. They were able to predict the measured parabolic eddy viscosity distribution in open channel flows by imposing a dissipation boundary condition at the free surface. The LES study of Shi et al. (2000) showed that as the free surface was approached, the vertical turbulence length scales decreased whereas the horizontal ones increased.

Nezu and Nakagawa (1993) stated that in the absence of shear stresses, the water surface can be approximated as a plane of symmetry, but the free surface's existence will reduce the turbulent length scale, as stated by Hunt (1984). This is also shown experimentally by Nezu and Nakagawa (1993). Since the length scale reduces and it is inversely proportional with the dissipation rate and directly proportional to $\mathrm{k}^{3 / 2}$, the dissipation near the free surface increases, as shown by Celik and Rodi (1984) in RANS and Komori et al. (1993) in DNS.

The conditions for Komori et al.'s (1993) DNS study of open channel flow are shown in Figure 1.2. In Figure 1.3(a), the DNS and laser Doppler velocimeter (LDV) results show that the vertical intensity decreases rapidly in the free surface region, while spanwise and streamwise intensities increase. Although a difference between the predictions and measurements is observed, the predictions explain rather well the 
measured intensities behavior. As shown in Figure 1.3 (b), the damping effect of the free surface is predicted in three DNS results.

Nakayama and Yokojima (2002) have studied open channel flows allowing free surface motion (see Figure 1.4). In Figure 1.5 (a\&b), comparing the LES results obtained with those obtained from DNS (Nakayama \& Yokojima, 2001), it is seen that the effects of free surface sub grid scale (SGS) terms in Smagorinsky modeling are not overwhelmingly important in subcritical Froude numbers, but they make some changes near the free surface. More on SGS modeling is presented in the next section.

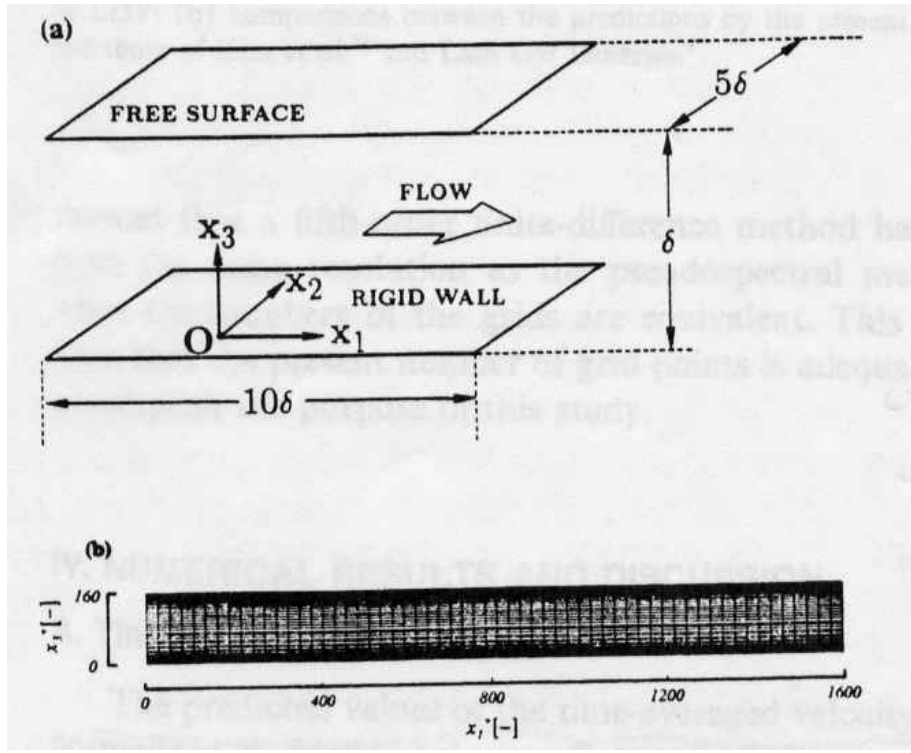

Figure 1.2. Geometry of the computational region: a) coordinate system b) mesh distribution (Komori et al., 1993) 

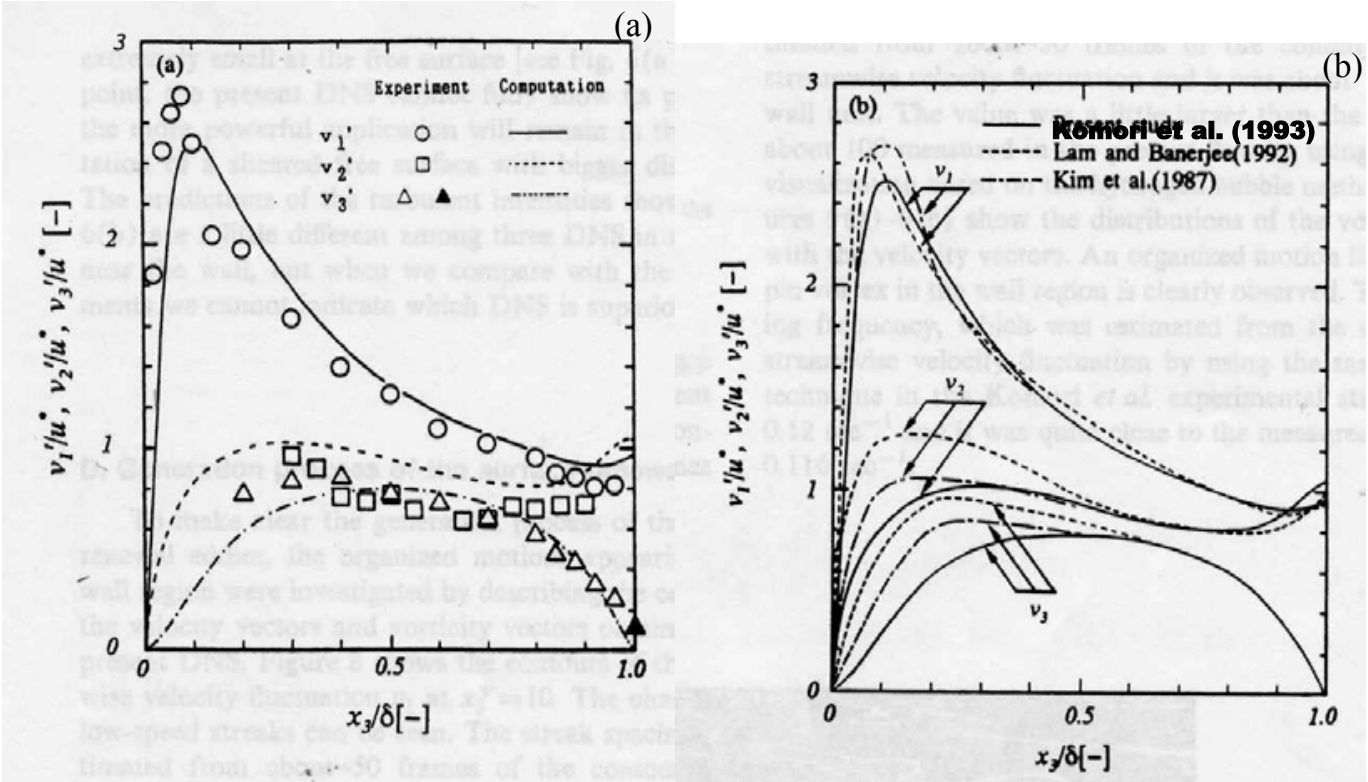

Figure 1.3. Turbulence intensity distributions: a) comparisons between predictions and measurement by LDV (Komori et al., 1990) b) comparisons between the predictions by Komori et al. (1993)'s DNS and those of Kim et al.(1987) and Lam and Banerjee (1992)

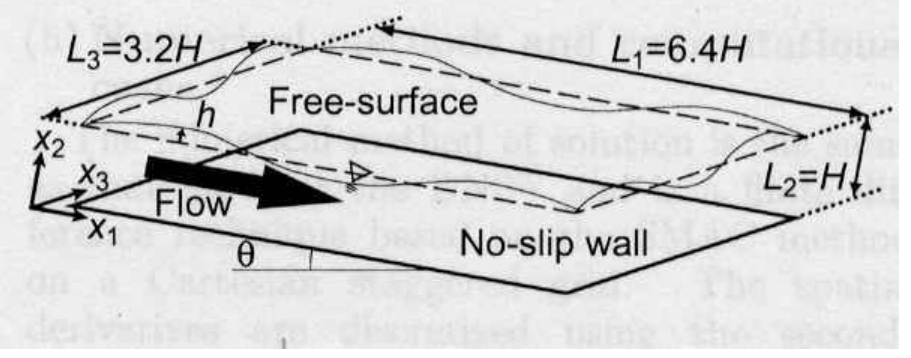

Figure 1.4. Geometry of the computational region having moving free surface (Nakayama and Yokojima, 2002) 


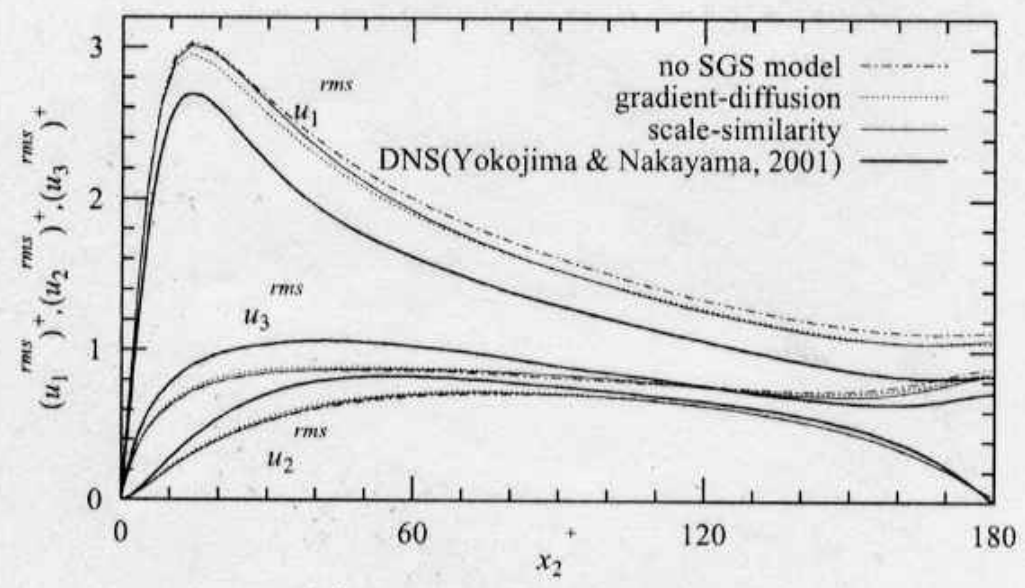

(c) Turbulence intensities

Figure 1.5. Turbulence intensity distributions: a) $\mathrm{Fr}=0.3$ b) $\mathrm{Fr}=0.6$ (Nakayama and Yokojima, 2002)

\subsubsection{SGS Studies}

The success of LES depends most critically on how accurate the modeling of the SGS stresses is. The models must represent the SGS stress in terms of the resolved field,. i.e. be capable of representing the effect of the unresolved motion essential in LES of turbulent flows.

Many SGS models have been developed in the last twenty years. Most of them are eddy viscosity models that use the Boussinesq hypothesis to calculate the eddy viscosity, that is the relationship between the SGS Reynolds stress and the strain rate of the large or resolved eddies is linear; i.e. 


$$
\tau_{i j}=-2 v_{t} \bar{S}_{i j}+\frac{2}{3} k_{s g s} \delta_{i j}=-v_{t}\left(\frac{\partial \bar{u}_{i}}{\partial x_{j}}+\frac{\partial \bar{u}_{j}}{\partial x_{i}}\right)+\frac{2}{3} k_{s g s} \delta_{i j}
$$

where $v_{\mathrm{t}}$ is the SGS eddy viscosity.

The simplest model has been proposed by Smagorinsky (1963), that is the basis of the many other advanced models, such as the scale similarity model proposed by Bardina et al. (1980), the dynamic sub-grid model (DSGS) proposed by Germano et al. (1991), the dynamic mixed model by Zang et al. (1993), and the dynamic two parameter model by Salvetti et al. (1997).

The Smagorinsky model uses a constant model coefficient for the entire flow domain and the eddy viscosity is expressed as,

$$
v_{t}=C_{s} \Delta^{2}\left(\bar{S}_{i j} \bar{S}_{i j}\right)^{1 / 2}
$$

where $\Delta$ is a characteristic length scale defined as $\Delta=\left(\Delta x_{1} \Delta x_{2} \Delta x_{3}\right)^{1 / 3}$ and $\mathrm{C}_{\mathrm{s}}$ is the Smagorinsky constant; here $\Delta \mathrm{x}_{1}, \Delta \mathrm{x}_{2}, \Delta \mathrm{x}_{3}$ represent the sides of a octahedral computational cell. Here, $\bar{S}_{i j}$ is the large scale (resolved) strain rate tensor, defined as, $\bar{S}_{i j}=\frac{1}{2}\left(\frac{\partial \bar{u}_{i}}{\partial x_{j}}+\frac{\partial \bar{u}_{j}}{\partial x_{i}}\right)$. The constant has been found to be a function of the filter width and the integral length scale of turbulence. $\mathrm{A} \mathrm{C}_{\mathrm{s}}$ value of 0.2 does a good job for isotropic turbulence, and for inhomogeneous flows this value must be reduced by half or more 
(Ferziger, 1993). McMillian et al. (1980) figured out that for homogeneous turbulence the value decreases with increasing strain rate. In the near wall region of channel flows, Moin and Kim (1982) did additional modifications to the Smagorinky model such that they used some damping functions to force the SGS stresses to vanish at the wall. On the other hand, the dynamic models provide a methodology to calculate an appropriate local value of the Smagorinsky constant. Germano et al. (1991) developed a new dynamic sub grid scale model, tested in fully developed turbulent channel flows, and obtained better results than those used the Smagorinsky model. Lilly (1992) modified this model. One of the main disadvantages of this model is the numerical instability because of the negative values and large variations of the coefficient (Sohankar et al., 2000).

While the above eddy viscosity models may be able to represent the global dissipative effects of the small scales, the details of the stresses and energy transfer can not be reproduced accurately (Piomelli, 1999). Scale similar and mixed models are alternative for this reproduction, by applying a second filter, having the same filter width, to the governing equations. The SGS stresses in the scale similar model can be written as,

$$
\tau_{i j}=-2 v_{t} S_{i j}+\left[\overline{\bar{u}_{i} \bar{u}_{j}}-\bar{u}_{i} \bar{u}_{j}\right\rfloor
$$

Scale similarity models require fine grid resolution. With decreasing grid resolution, it becomes more uncorrelated with the DNS results (Menon and Yeung, 1995). 
One-equation models in LES, using the analogy of one equation models in RANS (Wilcox, 1993), have been developed as a modified eddy viscosity model, where an additional transport equation is solved explicitly for a suitable parameter characterizing the turbulence, such as SGS turbulent kinetic energy. There is no assumption of local balance between SGS energy production and dissipation rate, however it has the drawback of specifying the length scale. When relatively coarse grids are considered (or high Reynolds number flows), the unresolved scales may contain energy-containing scales and a contribution of the sub-grid energy to the resolved SGS stresses may have to be explicitly computed (Menon and Kim, 1996). Models have been developed that employ a transport equation for the SGS kinetic energy, but require an empirical expression for the distribution of the turbulent length scale that appears in the model relations. In the literature many applications can be found: one equation models (Lewellen, 1977; Yoshiziwa and Horiuti, 1985; Menon and Yeung, 1995; Menon and Kim, 1996), dynamic one equation models (Ghosal et al., 1985, Krajnovic et al., 1999, Sohankar et al., 1999 and 2000).

So far, models with Boussinesq eddy viscosity approximation have been described. However, in some predictions of flow properties with this approximation, there are significant discrepancies from the corresponding measurements. There are some types of applications for which models based on this approximation may fail, such as flows with sudden change in the mean strain rate, flow over curved surfaces, flow in rotating and stratified fluids, and three dimensional flows (Wilcox, 1993).

The linear eddy viscosity can not represent the anisotropy of turbulence depending on the resolved scales (at some points the turbulence may become isotropic) 
observed near the free surface (Hamba, 2001). Some linear eddy viscosity models widely used for practical applications do not guarantee the non negativeness of the normal component of the Reynolds stresses (Hamba, 2001). However, the non-linear models can satisfy the realizibility constraints (Lumley, 1978): namely (i) the normal stress is nonnegative as the velocity is real, (ii) the model should satisfy the Schwarz' inequality for the shear stress and (iii) the determinant of the shear stress should be greater than or equal zero. In the literature, several nonlinear models have been proposed. Nisizima and Yoshizawa (1987) derived the model from statistical approaches, and applied it to square duct flows by producing reasonable results. Speziale (1987) derived the nonlinear constitutive model for anisotropic turbulence and applied it successfully to plane channel flow. The model requires the stresses depend at least quadratically on the mean velocity gradients. On the other hand, Speziale (1987) concluded that the $2^{\text {nd }}$ order nonlinear model should not have a quadratic vorticity term applied in a rotating frame because of the violation of realizibility. Hamba's (2001) model satisfies realizibility in a rotating frame. Rubinstein and Barton (1990) also suggested a nonlinear model and showed that they were able to predict anisotropic turbulent flows for high and low Reynolds numbers. There is another model that provides a measure of anisotropy, namely the k-e- $\mathrm{v}^{2}$ model of Durbin (1995). It seems to be a good model for computing wall bounded shear flows, such as confined swirling jets (Durbin, 1995) and other external flows (Kalitzin, 1999) with large regions of parabolic boundary layers. Because of the nature of the equations for the $\mathrm{k}-\mathrm{e}-\mathrm{v}^{2}$ model, the solution must be obtained by an iterative procedure. However, the nonlinear model proposed by Hamba (2001) does not require an iterative solution procedure. 


\subsection{Contributions from current work}

To the best of author's knowledge, there are no measurements nor LES studies in the literature focused on the wake behind a ship cruising on a circular track. This work constitutes the first application of a novel technique to the turning surface ship wake when the influence of the Coriolis force and centrifugal forces on turbulence generation is accounted for.

The second area of contribution is the comparative investigation of the physics of turbulent wakes aft of a turning ship and a ship cruising on a straight course: wake spreading, turbulence activity, and wake structures are studied using LES and results are compared to experimental observations from literature. It is demonstrated that LES is capable of predicting complex turbulent flow features in detail.

In large eddy simulation, the key to the reliability of the solution with relatively large mesh size (this is unavoidable for high Re flows) is to develop effective and physically correct subgrid-scale (SGS) models. The behavior of various SGS models in LES of free surface turbulent flows is investigated. The anisotropy of the turbulence observed near a free surface can not be obtained by a standard Smagorinsky model (SMG). The SMG model is improved with free surface modifications. This model is verified on an open channel flow benchmark and then applied to the surface ship wake. Then, a nonlinear one-equation model is formulated and applied to the open channel flow. The new non linear SGS model improves the turbulent predictions near the free surface for open channel flow. The performance of existing and improved SGS models is 
compared and discussed. This study provides a better understanding of SGS models, when applied to the case of the wake of a non turning and turning surface ship. 


\section{Chapter 2}

\section{LITERATURE REVIEW}

In this chapter, the time-dependent, volume averaged, three-dimensional NavierStokes equations that govern the physics of turbulent flow are presented. The main focus of this study is on the turbulent flow dynamics in the wake of a ship cruising on a circular track in which the Coriolis and centrifugal forces are of importance because of the noninertial reference frame used. These forces are accounted for by including additional source terms into the momentum equations. Then, the LES scheme is briefly described. To tackle problems of complex geometries, the equations are transformed from the physical to the computational space. Essential information on the numerical method is introduced and the verification of the whole numerical procedure previously performed by Shi (2001) is briefly described. 


\subsection{Governing Equations and Navier Stokes solver}

The LES code used was originally developed by Zang et al. (1994). The equations for an incompressible, viscous fluid flow in Cartesian (physical) space can be presented in terms of the Cartesian velocities $u_{j}$ as

$$
\begin{gathered}
\frac{\partial u_{j}}{\partial x_{j}}=0 \\
\frac{\partial u_{i}}{\partial t}+\frac{\partial\left(u_{i} u_{j}\right)}{\partial x_{j}}=-\frac{1}{\rho} \frac{\partial P}{\partial x_{i}}+v \frac{\partial^{2} u_{i}}{\partial x_{j}^{2}}+2 \Omega_{S}\left(u_{1} \frac{\partial x_{3}}{\partial x_{i}}-u_{3} \frac{\partial x_{1}}{\partial x_{i}}\right)+\Omega_{s}^{2} x_{i}\left(1-\delta_{i 3}\right)(2.2
\end{gathered}
$$

An additional equation that represents the conservation of a scalar, such as kinetic energy, temperature, etc. is,

$$
\frac{\partial \phi}{\partial t}+\frac{\partial\left(\phi u_{j}\right)}{\partial x_{j}}=\alpha \frac{\partial^{2} \phi}{\partial x_{j}^{2}}+S_{i}(\phi)
$$

where $\alpha$ is a material coefficient that could be thermal diffusivity, conductivity or viscosity, depending on which scalar equation is solved. $S_{i}(\phi)$ is a sink/source term. In the above equations, $\mathrm{u}_{\mathrm{j}}$ is the Cartesian velocity vector, $\mathrm{P}$ is the total pressure, and $\mathrm{i}, \mathrm{j}, \mathrm{k}$, are the notations that represent the directions; $\mathrm{x}_{1}$ is the axial coordinate, $\mathrm{x}_{2}$ is the vertical 
coordinate, and $\mathrm{x}_{3}$ is the transverse coordinate in the Cartesian coordinate system. $\Omega_{\mathrm{s}}$ is the angular velocity of the system rotation due to the turning of the ship, $v$ is the kinematic viscosity and $\delta_{\mathrm{ij}}$ is the Kronecker delta symbol. In Eqn. 2.2, third term in the right hand side (RHS) represents the Coriolis force and the fourth term represents the Centrifugal force due to the turning of the ship. It should be noted that Einstein summation rule applies to repeated indices except for the term, $\Omega_{s}{ }^{2} x_{i}\left(1-\delta_{i 3}\right)$.

The Navier Stokes equations have been developed for a general purpose model of fluid flow from basic principles of conservation of mass and momentum for a Newtonian fluid. Moreover, the numerical models, such as finite volume method (Ferziger et al., 1997) also incorporate the law of conservation of mass and momentum for space integration.

The filtering process plays an important role in distinguishing small scales and large scales in LES. A flow variable, f can be decomposed into a large scale of the flow field component that is resolved, $\bar{f}$ and a small scale component that is filtered out, $f^{\prime}$, as,

$$
f=\bar{f}+f^{\prime}
$$

Then the resolved scale field is obtained by applying spatial filtering that can be generally expressed by the convolution integral (Leonard, 1974) for the calculation domain, D, as, 


$$
\bar{f}\left(x_{1} x_{2} x_{3}\right)=\int_{D} \prod_{j=1}^{3} G_{j}\left(x_{j}, x_{j}^{\prime}: \bar{\Delta}\right) f\left(x_{1}^{\prime} x_{2}^{\prime} x_{3}^{\prime}\right) d x_{1}^{\prime} d x_{2}^{\prime} d x_{3}^{\prime}
$$

where $\mathrm{G}$ is the filter function and $\bar{\Delta}$ is the filter width, i.e. the wavelength of the smallest scale retained by the filtering function. The most commonly used filter functions are the box filter, the sharp Fourier cutoff filter and Gaussian filter (best defined in wave space), and the top hat filter (in real space) (Piomelli, 1999). In the present finite volume formulation, a volume average box filter used by Deardoff (1970), is used, in which $\mathrm{G}_{\mathrm{j}}=1$ (Zang, 1993).

Applying the filtering operator to the governing equations and following the formulation of Zang et al. (1993) in a conservative manner, the spatially filtered flow conservation equations can be written as,

$$
\begin{gathered}
\frac{\partial \bar{u}_{j}}{\partial x_{j}}=0 \\
\frac{\partial \bar{u}_{i}}{\partial t}+\frac{\partial \bar{F}_{i j}}{\partial x_{j}}=\bar{S}_{i} \\
\frac{\partial \bar{\phi}}{\partial t}+\frac{\partial \bar{R}_{j}}{\partial x_{j}}=\bar{S}_{i}(\phi)
\end{gathered}
$$

where 


$$
\begin{aligned}
& \bar{F}_{i j}=\bar{u}_{i} \bar{u}_{j}+\bar{p} \delta_{i j}-v \frac{\partial \bar{u}_{i}}{\partial x_{j}}+\tau_{i j} \\
& \bar{S}_{i}=2 \Omega_{S}\left(\bar{u}_{1} \frac{\partial x_{3}}{\partial x_{i}}-\bar{u}_{3} \frac{\partial x_{1}}{\partial x_{i}}\right) \\
& \bar{R}_{j}=\bar{u}_{j} \bar{\phi}-\alpha \frac{\partial \bar{\phi}}{\partial x_{j}}+\chi_{j}
\end{aligned}
$$

Here, both, the SGS stress, $\tau_{i j}$ and the SGS flux, $\chi_{j}$ represent the effect of the SGS motion. They arise due to the filtering of the nonlinear advective terms. The formulations are,

$$
\begin{aligned}
& \tau_{i j}=\overline{u_{i} u_{j}}-\bar{u}_{i} \bar{u}_{j} \\
& \chi_{j}=\overline{u_{j} \phi}-\bar{u}_{j} \bar{\phi}
\end{aligned}
$$

Hence, the SGS stress and the SGS flux both contain the interaction of subgrid scales with themselves and with the resolved scales. In the above equations, $\bar{u}_{j}$ is the filtered velocity vector and $\mathrm{p}$ is the reduced dynamic pressure in which the total pressure, $\mathrm{P}$, is calculated as;

$$
P=\rho_{o} p+\frac{1}{2} \rho_{o} \Omega_{s}^{2} r^{2}
$$


where $r^{2}$ is the square of the distance to the rotation axis, in terms of both the axial and the transverse directions, i.e. $r^{2}\left(=x_{1}^{2}+x_{3}^{2}\right)$ and $\rho_{\mathrm{o}}$ is the reference density. It should be noted that the non-inertial effects are split with Coriolis terms appearing as a source term in Equation 2.7 and since the centrifugal force term is independent of the fluid motion, the effect of centrifugal force is included in the total pressure term, i.e. Equation 2.14.

Since the discrete solution represents the resolved field which is topped by an overbar computed, thus the stress and the flux terms should be modeled using the resolved quantities. Most SGS models for $\tau_{\mathrm{ij}}$ and $\chi_{j}$ are eddy viscosity models of the form:

$$
\begin{gathered}
\tau_{i j}-\frac{\delta_{i j}}{3} \tau_{k k}=-2 v_{t} \bar{S}_{i j}+C_{r}\left(L_{i j}^{m}-\frac{\delta_{i j}}{3} L_{k k}^{m}\right) \\
\chi_{j}=-\alpha_{T} \frac{\partial \bar{\phi}}{\partial x_{j}}+C_{r} P_{j}
\end{gathered}
$$

where $v_{t}$ is the turbulent eddy viscosity, it represents the effects of sub grid turbulence; in our case, $\alpha_{t}$ is the turbulence diffusivity and $\bar{S}_{i j}$ is the large scale (resolved) strain rate tensor, defined as, 


$$
\bar{S}_{i j}=\frac{1}{2}\left(\frac{\partial \bar{u}_{i}}{\partial x_{j}}+\frac{\partial \bar{u}_{j}}{\partial x_{i}}\right)
$$

Eq. 2.15 and 2.16 introduces two sets of additional terms to the filtered governing equations: $\mathrm{C}_{\mathrm{r}}$ and $L^{m}{ }_{i j} ; \mathrm{C}_{\mathrm{r}}$ and $\mathrm{P}_{\mathrm{j}} . L^{m}{ }_{i j}$, the modified Leonard term and $\mathrm{P}_{\mathrm{j}}$ are defined by,

$$
\begin{gathered}
L_{i j}^{m}=\overline{\bar{u}_{i} \bar{u}_{j}}-\overline{\overline{u_{i} u_{j}}} \\
P_{j}=\overline{\overline{u_{i}} \bar{\phi}}-\overline{\overline{u_{i}}} \overline{\bar{\phi}}
\end{gathered}
$$

The modified Leonard term or $\mathrm{P}_{\mathrm{j}}$ represent the interactions between resolved scales that result in sub-grid scale contributions and can be computed directly from the resolved flow field (Piomelli, 1999). The value of the scale similarity coefficient, $\mathrm{C}_{\mathrm{r}}$ in Eqn. 2.15 is either 0 or 1 or may be determined dynamically depending on the type of sub-grid scale (SGS) model being used. When $\mathrm{C}_{\mathrm{r}}=0$, Eqn. 2.15 represents the Smagorinsky model. When $\mathrm{C}_{\mathrm{r}}=1$, it represents the dynamic mixed model of Zang (1993).

To tackle problems of complex geometries, the above mentioned equations are transformed from the physical to computational space and formulated for a generalized curvilinear coordinate system. The solution of numerical problems in complex domains using boundary-fitted curvilinear coordinates is now a typical technique. The physical 
space is denoted by coordinates $\left(x_{1}, x_{2}, x_{3}\right)$ and the computational space by $\left(\xi_{1}, \xi_{2}, \xi_{3}\right)$. The chain rule of derivatives

$$
\frac{\partial}{\partial x_{j}}=\frac{\partial \xi_{z}}{\partial x_{j}} \frac{\partial}{\partial \xi_{z}}
$$

has been applied.

In order to use the finite volume discretization, it is desirable to cast the equations in the "Strong-Conservation-Law Form" as explained briefly in Zang (1993).

Substituting Eqn. 2.15 into Eqn. 2.7, and applying coordinate transformation and combining terms accordingly, Eqs. 2.6, 2.7 and 2.8 in time-dependent boundary-fitted curvilinear coordinates are,

$$
\begin{gathered}
\frac{\partial \bar{U}_{m}}{\partial \xi_{m}}=0 \\
\frac{\partial\left(J^{-1} \bar{u}_{i}\right)}{\partial t}+\frac{\partial \bar{F}_{i m}}{\partial \xi_{m}}=\bar{S}_{i} \\
\frac{\partial\left(J^{-1} \bar{\phi}\right)}{\partial t}+\frac{\partial \bar{R}_{m}}{\partial \xi_{m}}=\bar{S}_{T i}(\phi)
\end{gathered}
$$

where 


$$
\begin{gathered}
\bar{F}_{i m}=\bar{U}_{m} \bar{u}_{i}+J^{-1} \frac{\partial \xi_{m}}{\partial x_{i}} \bar{p}-\left(v+v_{T}\right) G^{m n} \frac{\partial \bar{u}_{i}}{\partial \xi_{n}} \\
\bar{S}_{i}=2 J^{-1} \Omega_{s} \frac{\partial \xi_{m}}{\partial x_{i}}\left(\bar{u}_{1} \frac{\partial x_{3}}{\partial \xi_{m}}-\bar{u}_{3} \frac{\partial x_{1}}{\partial \xi_{m}}\right)+ \\
J^{-1} \frac{\partial \xi_{m}}{\partial x_{j}} \frac{\partial \xi_{n}}{\partial x_{i}} \frac{\partial v_{T}}{\partial \xi_{m}} \frac{\partial \bar{u}_{j}}{\partial \xi_{n}}-C_{r} \frac{\partial}{\partial \xi_{m}}\left(J^{-1} \frac{\partial \xi_{m}}{\partial x_{j}} L_{i j}^{m}\right) \\
\bar{R}_{m}=\bar{U}_{m} \bar{\phi}-\left(\alpha+\alpha_{T}\right) G^{m n} \frac{\partial \bar{\phi}}{\partial \xi_{n}} \\
\bar{S}_{T i}(\phi)=\bar{S}_{i}^{\prime}(\phi)-C_{r} \frac{\partial}{\partial \xi_{m}}\left(J^{-1} \frac{\partial \xi_{m}}{\partial x_{j}} P_{j}\right)
\end{gathered}
$$

where for the curvilinear space variables; the inverse Jacobian, defined as $J^{-1}=\operatorname{det}\left|\frac{\partial x_{i}}{\partial \xi_{m}}\right| ;$ the contravariant velocity , $\bar{U}_{m}=J^{-1} \frac{\partial \xi_{m}}{\partial x_{j}} \bar{u}_{j}$; the contravariant volume metrics, $G^{m n}$, that measures the skewness of a grid cell, is defined as $G^{m n}=J^{-1} \frac{\partial \xi_{m}}{\partial x_{j}} \frac{\partial \xi_{n}}{\partial x_{j}}$

. $\bar{S}_{i}^{\prime}(\phi)$, the transformation of $\bar{S}_{i}(\phi)$ to the computational domain, must be changed accordingly, depending on which scalar equation is solved.

If the flux terms $\partial \bar{F}_{i m} / \partial \xi_{m}$ and $\partial \bar{R}_{m} / \partial \xi_{m}$ are split like in Zang (1993) as,

$$
\frac{\partial \bar{F}_{i m}}{\partial \xi_{m}}=-\left[C_{i}+B_{i}(p)+D_{E}\left(u_{i}\right)+D_{I}\left(u_{i}\right)\right]
$$




$$
\frac{\partial \bar{R}_{m}}{\partial \xi_{m}}=-\left[E_{\rho}+F_{E}(\rho)+F_{I}(\rho)\right]
$$

where $C_{i}$ and $E_{\rho}$ represent the convective terms, $B_{i}$ is the discrete operator for the pressure gradient term, $\mathrm{D}_{\mathrm{E}}$ and $\mathrm{D}_{\mathrm{I}}\left(\mathrm{F}_{\mathrm{E}}\right.$ and $\left.\mathrm{F}_{\mathrm{I}}\right)$ are discrete operators for the explicitly treated off diagonal terms and the implicitly treated diagonal viscous (diffusive) terms.

\subsection{Numerical Method}

The computer code is based on an essentially non-staggered grid, finite volume method using a fractional time step approach. A staggered grid method in curvilinear coordinates requires a large amount of computer memory for the metrics (Zang, 1993), hence the non-staggered method originally developed by Rhie and Chow (1983) has been used to avoid these kind of difficulties. Cartesian variables such as velocity and pressure are stored at cell centers whereas the contravariant volume fluxes are defined at cell faces in a manner analogous to the staggered-mesh system. The volume fluxes are not solution variables, but rather are determined through interpolation of the cell-centered velocity values plus a projection operation that guarantees exact conservation of mass. A traditional non staggered method does not enforce mass conservation in the cell and causes the pressure field to decouple (it produces spurious oscillations in the pressure field, i.e."checkerboard" pattern) (Zang et al. 1994), whereas the method of Rhie and Chow (1983) prevents the decoupling in its structure by defining the volume flux on its corresponding face of the cell in addition to the Cartesian velocity components at the cell 
center, therefore the momentum and continuity are both enforced in the same control volume and the solutions are free from spurious pressure oscillations. It is directly applicable to curved domains, as the accuracy of the method is not affected by grid orientations because of the non-staggered grid layout. However, this process eliminates odd-even decoupling at the cost of introducing implicit $4^{\text {th }}$ order dissipation, which in turn may affect mass conservation (Paterson, 2003).

Non-orthogonal curvilinear coordinates are applied with an overall second order accuracy in both space and time. The Crank-Nicolson discretization scheme has been applied for diagonal viscous $\left(\mathrm{D}_{\mathrm{I}}\right)$ and diffusion $\left(\mathrm{F}_{\mathrm{I}}\right)$ terms in order to remove the viscous instability (Zang et al., 1994) while an explicit Adams-Bashforth scheme is employed for all the other terms. The off diagonal viscous terms $\left(\mathrm{D}_{\mathrm{E}}\right)$ are treated explicitly in order to simplify the LHS matrix of the momentum equation. The result like in Zang (1993) is

$$
\begin{gathered}
\frac{\delta \bar{U}_{m}}{\delta \xi_{m}}=0 \\
J^{-1 \frac{\left(\bar{u}_{i}^{n+1}-\bar{u}_{i}^{n}\right)}{\Delta t}=}=\frac{3}{2}\left[C_{i}^{n}+D_{E}\left(\bar{u}_{i}^{n}\right)+\bar{S}_{i}^{n}\right]-\frac{1}{2}\left[C_{i}^{n-1}+D_{E}\left(\bar{u}_{i}^{n-1}\right)+\bar{S}_{i}^{n-1}\right] \\
+\mathrm{B}_{\mathrm{i}}\left(\bar{p}^{n+1}\right)+\frac{1}{2}\left[D_{I}\left(\bar{u}_{i}^{n+1}+\bar{u}_{i}^{n}\right)\right] \\
J^{-1} \frac{\left(\bar{\rho}_{i}^{n+1}-\bar{\rho}_{i}^{n}\right)}{\Delta t}=\frac{3}{2}\left[E_{\rho}^{n}+F_{E}\left(\bar{\rho}^{n}\right)+\bar{S}_{\rho}^{n}\right]-\frac{1}{2}\left[E_{\rho}^{n-1}+F_{E}\left(\bar{\rho}^{n-1}\right)+\bar{S}_{\rho}^{n-1}\right] \\
+\frac{1}{2}\left[F_{I}\left(\bar{\rho}_{i}^{n+1}+\bar{\rho}_{i}^{n}\right)\right]
\end{gathered}
$$


where $\delta / \delta \xi_{m}$ represents discrete finite difference operators in the computational space, superscripts represent the time step, $C_{i}$ represents the convective terms, $B_{i}$ represents the dicrete operator for the pressure gradient terms.

The central differencing (CD) scheme (with special care due to numerical instabilities) or Quadratic Upstream Interpolation for Convective Kinematics (QUICK) that calculates the face value from the nodal values using a quadratic upwind interpolation is applied to discretize the convective terms $\left(\mathrm{C}_{\mathrm{I}}\right)$. The accuracy of QUICK has been compared to $\mathrm{CD}$, the $1^{\text {st }}$ order upwind scheme, a hybrid scheme, with the result obtained that QUICK produced good results (Zang, 1993, Hayasa, 1999). Theoretically the higher the order of the numerical scheme, the better the resolution should be under the same grid spacing (Shi, 2001). However, Rai and Moin (1991) have shown that higher order of accuracy combined with coarse grid spacing does not necessarily give better results. Jordan (1999) showed that the results can be improved by improving the grid spacing. The power law scheme is inaccurate under some limitations, such as when convection is dominant; it reduces to $1^{\text {st }}$ order upwind scheme (Patankar, 1980). On the other hand, higher order CD schemes have in addition the problem of artificial high frequency oscillations that may contaminate the turbulence field (Rai and Moin, 1991). In LES, explicit schemes are preferable, but if stability is an issue, some implicitness can be introduced, i.e. Crank-Nicolson time splitting.

The spatial derivates are computed by $2^{\text {nd }}$ order central differences in the momentum equations. Only the convective term in the scalar equation $\left(\mathrm{E}_{\rho}\right)$ is discretized using the SHARP scheme (Leonard, 1988) since it is computationally more expensive than QUICK. 
Because there is no explicit equation to solve for the pressure in time, the fractional step method is applied to solve the incompressible Navier-Stokes equation. The fractional step approach (Kim and Moin, 1985) or projection method, basically a three step predictor corrector method, splits the numerical operators and achieves velocitypressure decoupling. The intermediate velocities are interpolated onto the faces of the control volume to form the source terms of the pressure Poisson equation. The pressure field is obtained by solving the pressure Poisson equation iteratively with a multigrid method (Brandt, 1977). The true velocity field is then obtained by correcting the predicted velocity with pressure. The steps are summarized from Zang (1993) as,

1. Predictor step:

$$
\begin{aligned}
& \left(I-\frac{\Delta t}{2 J^{-1}} D_{I}\right)\left(u_{i}^{*}-\bar{u}_{i}^{n}\right)= \\
& \quad \frac{\Delta t}{J^{-1}}\left\{\frac{3}{2}\left[C_{i}^{n}+D_{E}\left(\bar{u}_{i}^{n}\right)+\bar{S}_{i}^{n}\right]-\frac{1}{2}\left[C_{i}^{n-1}+D_{E}\left(\bar{u}_{i}^{n-1}\right)+\bar{S}_{i}^{n-1}\right]+D_{I}\left(\bar{u}_{i}^{n}\right)\right\}
\end{aligned}
$$

2. Computing the pressure field, i.e. finding $\phi$;

If the corrector step of the fractional step method (Equation 2.37) to the Cartesian velocity components defined on a certain face of the control volume,

$$
\bar{u}_{i}^{n+1}=u_{i}^{*}-\Delta t\left(\frac{\delta \xi_{m}}{\delta x_{i}} \frac{\delta \phi^{n+1}}{\delta \xi_{m}}\right)
$$


Combining $\bar{U}_{m}=J^{-1} \frac{\partial \xi_{m}}{\partial x_{j}} \bar{u}_{j}$ with Equation 2.34, the equations for $\bar{U}_{m}^{n+1}$,

$$
\bar{U}_{m}^{n+1}=U_{m}^{*}-\Delta t\left(G^{m n} \frac{\delta \phi^{n+1}}{\delta \xi_{m}}\right)
$$

where $U_{m}^{*}=J^{-1} \frac{\delta \xi_{m}}{\delta x_{j}} u_{j}^{*}$ is called the intermediate volume flux. Since the intermediate velocity $u_{j}^{*}$ is defined at the cell center, while the fluxes $U_{m}^{*}$ and $\bar{U}_{m}^{n+1}$ are defined on the cell faces, $u_{j}^{*}$ has to be interpolated onto the cell faces in order to compute $U_{m}^{*}$.

By substituting Equation 2.35 into Equation 2.30, the pressure passion equation for $\phi^{n+1}$ is obtained as,

$$
\frac{\delta}{\delta \xi_{m}}\left(G^{m n} \frac{\delta \phi^{n+1}}{\delta \xi_{m}}\right)=\frac{1}{\Delta t}\left(\frac{\delta U_{m}^{*}}{\delta \xi_{m}}\right)
$$

3. Corrector step:

$$
\bar{u}_{i}^{n+1}-u_{i}^{*}=\frac{\Delta t}{J^{-1}}\left[B_{i}\left(\phi^{n+1}\right)\right]
$$


where $I$ is the identity matrix, $\mathrm{u}_{\mathrm{i}}{ }^{*}$ is the intermediate velocity and the scalar $\phi$ is related to the pressure $\bar{p}$ by

$$
B_{i}(\bar{p})=\left(J^{-1}-\frac{\Delta t}{2} D_{I}\right)\left(\frac{B_{i}(\phi)}{J^{-1}}\right)
$$

Detailed information can be found in Zang (1993).

\subsection{Subgrid scale models}

SGS stresses that are modeled represent the effects of the sub-grid scale motion on the resolved motion in that they dissipate the resolved energy or backscatter energy to the resolved eddies. To predict the flow dynamics of the wake behind a turning ship, the standard Smagorinksy model (Smagorinksy, 1963), based on Boussinesq eddy viscosity hypothesis, has been used. This model is developed by assuming that the small scales are in equilibrium so that energy production and dissipation are in balance, moreover the small scales dissipate all the energy they receive from the resolved scales. This assumption is made to simplify the phenomena and the algebraic model for the eddy viscosity is, 


$$
v_{t}=C_{s} \Delta^{2}\left(\bar{S}_{i j} \bar{S}_{i j}\right)^{1 / 2}
$$

where the filter length scale, $\Delta$ is the volume average box filter used by Deardoff (1970) usually calculated as the geometric average of mesh spacings in the Cartesian directions, defined as $\Delta=\left(\Delta x_{1} \Delta x_{2} \Delta x_{3}\right)^{1 / 3}$ in finite volume formulations (especially for anisotropic grids) and $\mathrm{C}_{\mathrm{s}}$ is the Smagorinsky constant. This constant is determined from the isotropic turbulence decay. It is interesting to note that the filtering process that's applied through control volume approach may use a significantly different length scales in different directions due to grid stretching. To see the influence of grid aspect ratio on filtering process, a model equation is used and different filter lengths have been applied and results have been shown in Appendix B. The results show that the grid size in each direction influences mostly the degree of filtering in that direction; the influence on the other direction is much lesser. In Chapter 3, the application of Smagorinksy model to the wake behind a turning ship wake will be briefly presented.

To emphasize the effects of the free surface fluctuation, a new grid filter width, $\Delta^{*}$ will be introduced, such as

$$
\frac{1}{\left(\Delta^{*}\right)^{n}}=\frac{1}{l^{n}}+\frac{1}{\Delta^{n}}
$$


In Equation 2.40, a length scale function similar to that introduced by Mason and Thomson (1992) is used. The Smagorinsky model only involves a single grid filter, $\Delta$. However, the characteristic of the near free surface region is that the length scale of the subgrid scale motion will not be given by a constant value of $\Delta$, but will decrease as the free surface is approached. Therefore, the above simple and arbitrary length scale function is selected to match between the near surface and the interior, where the value of $\mathrm{n}$ is allowed to vary and $l$ is the turbulent characteristic length scale.

The free surface suppresses the vertical movement of eddies, therefore the turbulent Reynolds number, $\mathrm{Re}_{\mathrm{t}}$ approaches to zero as $\operatorname{Re}_{t}=\frac{v^{\prime} y}{v}$. On the other hand, Van Driest (1956) had successfully derived a model to account for the damping of turbulence caused by molecular viscosity action near the solid surface. Following the same line of physical reasoning, the motion near the free surface is damped in a semiinfinite viscous liquid (Ueda et al., 1977). Therefore, to obtain this damping effect of the free surface on $v_{t}$, one can formulate $l$ like a Van Driest model,

$$
l=\kappa y_{s}\left(1-e^{-\left(y_{s}^{+} / 26\right)^{a}}\right)
$$

where the coefficient " $a$ " can be arbitrarily chosen according to experimental data. For this study, " $a$ " was chosen to be 3 to match the eddy viscosity distribution for an open channel flow in Nezu and Nakayagawa (1993). Here, $y_{\mathrm{s}}$ is the distance from the free surface and $\kappa$ is the Von Karman constant. 
In Equation 2.41, the length parameter $\mathrm{y}_{\mathrm{s}}+$ can be obtained using the velocity scale, $u$ at the point nearest to the free surface as,

$$
\begin{aligned}
& u=\sqrt{\frac{\tau_{s}}{\rho}}=\sqrt{2 v_{t} \bar{S}_{12}} \\
& y_{s}^{+}=\frac{y_{s} u}{v}=\frac{y_{s}}{v} \sqrt{2\left(C_{s} \Delta^{2} \bar{S}\right) \bar{S}_{12}}
\end{aligned}
$$

where

$$
\begin{aligned}
& \bar{S}=\left(\bar{S}_{i j} \bar{S}_{i j}\right)^{1 / 2} \\
& \bar{S}_{12}=\text { the resolved strain rate at a location near the free surface }
\end{aligned}
$$

Hence, the new model, referred to "l-modeling", will then be rewritten as,

$$
v_{t}=C_{s}\left(\Delta^{*}\right)^{2} \bar{S}
$$

It should be stated that in near wall applications, the original Van Driest damping function has also been used and the preliminary results of the application of 1-modeling to the wake behind a turning ship will be briefly presented in Chapter 3 .

However, these two models are not suitable for complex flows as they use constant eddy viscosity coefficients. As stated in Chapter 1, one-equation SGS models have many advantages, such as solving for the transport of the sub-grid kinetic energy 
explicitly. On the other hand, for some predictions of flow properties with the Boussinesq eddy viscosity approximation, there are deficiencies in comparison to measurements. Since a three dimensional turning ship wake application with rotational effects is being simulated, the Boussinesq eddy viscosity approximation is not expected to perform well. The linear eddy viscosity models widely used for practical applications, being isotropic, can not represent the anisotropy of turbulence observed near the free surface (Hamba, 2001). This anisotropy may become important for complex flows containing large length scales. It should be emphasized one more time here that by the linear eddy viscosity representation, the eddy viscosity models do not guarantee the non-negativeness of the normal component of the Reynolds stresses which is an unphysical behavior. A non linear SGS turbulence model can be used to rectify these short comings of the linear eddyviscosity models.

In a Reynolds stress model, the Reynolds stress is determined by transport equations. Therefore the subgrid scale kinetic energy, ksgs that needs to be solved in the one equation model is as follows:

$$
\frac{\partial k_{s g s}}{\partial t}+\frac{\partial}{\partial x_{j}}\left(\bar{u}_{j} k_{s g s}\right)=\tau_{i j} \frac{\partial \bar{u}_{j}}{\partial x_{j}}-\varepsilon_{s g s}+\frac{\partial}{\partial x_{j}}\left[\left(v_{t}+v\right) \frac{\partial k_{s g s}}{\partial x_{j}}\right]
$$

Using an analogy to the k- $\varepsilon$ turbulence model of Hamba (2001), a new model for the above Reynolds stress term, $\tau_{\mathrm{ij}}$, that is algebraically related to the mean velocity field will be proposed in the form, 


$$
\tau_{i j}=\tau_{i j}(k, \varepsilon, \nabla \bar{u})
$$

where components of tensor $\tau$ are functions of $\mathrm{k}, \varepsilon$ and the deformation rate tensor, $(\nabla \bar{u})_{k l}\left(=\partial \bar{u}_{k} / \partial x_{l}\right)$. In Hamba (2001) the proposed form (Eq. 2.45) has been proven to satisfy the first realizability constraint. More, Hamba (2001) has stated that if the first condition is satisfied, then the other two conditions are automatically ensured, however the proposed model has been proven to satisfy the second realizability constraint, the Schwarz' inequality.

The two-scale direct interaction approximation (TSDIA) has been used to derive the nonlinear one equation model just like deriving the nonlinear $\mathrm{k}-\varepsilon$ turbulence model of Hamba (2001). As an advantage, since the model is derived analytically, it can satisfy the realizability for an arbitrary turbulent flow (Hamba, 2001). The details of the derivation can be found in Hamba (2001).

Then, a realizable model theoretically derived for Reynolds stress, $\tau_{\mathrm{ij}}$ in the one equation model (Eq. 2.44) in terms of the strain rate and vorticity tensor is formulated as follows,

$$
\begin{aligned}
\tau_{i j}= & \frac{2}{3} k_{s g s} \delta_{i j}-2 v_{t} \bar{S}_{i j}+N_{1}\left(\bar{S}_{i k} \bar{S}_{k j}-\frac{1}{3} \bar{S}^{2} \delta_{i j}\right)+N_{2}\left(\bar{S}_{i k} \bar{W}_{k j}+\bar{S}_{j k} \bar{W}_{k i}\right)+ \\
& N_{3}\left(\bar{W}_{i k} \bar{W}_{k j}+\frac{1}{3} \bar{W}^{2} \delta_{i j}\right)
\end{aligned}
$$


where turbulent eddy viscosity can be modeled as,

$$
v_{t}=C_{v 1} f \frac{k_{s g s}^{2}}{\varepsilon}
$$

and

$$
\begin{aligned}
& N_{1}=C_{v 2} f \frac{k_{s g s}^{3}}{\varepsilon^{2}} \\
& N_{2}=C_{v 3} f \frac{k_{s g s}^{3}}{\varepsilon^{2}} \\
& N_{3}=C_{v 4} f \frac{k_{s g s}^{3}}{\varepsilon^{2}} \\
& f=\left[1+C_{v 5} \frac{k_{s g s}^{2}}{\varepsilon^{2}} S^{2}+C_{v 6} \frac{k_{s g s}^{2}}{\varepsilon^{2}} W^{2}\right]^{-1}
\end{aligned}
$$

where

$$
\bar{S}_{i j}=\text { strain rate tensor }=\frac{1}{2}\left(\frac{\partial \bar{u}_{i}}{\partial x_{j}}+\frac{\partial \bar{u}_{j}}{\partial x_{i}}\right) \quad ; \quad \bar{S}^{2}=\bar{S}_{i j} \bar{S}_{i j}
$$




$$
\bar{W}_{i j}=\text { vorticity tensor }=\frac{1}{2}\left(\frac{\partial \bar{u}_{i}}{\partial x_{j}}-\frac{\partial \bar{u}_{j}}{\partial x_{i}}\right) \quad ; \quad \bar{W}^{2}=\bar{W}_{i j} \bar{W}_{i j}=-\bar{W}_{i j} \bar{W}_{j i}
$$

The model constants (Hamba, 2001) are chosen to be

$$
\begin{aligned}
& C_{v 1}=\frac{7}{360 \pi C_{\sigma} C_{w}} ; C_{v 2}=\frac{1360}{343} C_{v 1}^{2} \\
& C_{v 3}=-\frac{160}{49} C_{v 1}^{2} ; C_{v 4}=-\frac{80}{49} C_{v 1}^{2} \\
& C_{v 5}=\frac{20}{7} C_{v 1}^{2} ; C_{v 6}=\frac{100}{49} C_{v 1}^{2}
\end{aligned}
$$

Here, realizability is satisfied for a value of $C_{v 1}=0.123$ if the values $C_{\sigma}=0.12$ and $\mathrm{C}_{\mathrm{w}}=0.42$ are taken from TSDIA (Hamba, 2001). By retaining $\mathrm{W}^{2}$ in the $\mathrm{f}$ function, the realizability is also satisfied even in a rotating frame (Hamba, 2001). The values of the model constants in Equations (2.54-2.56) are obtained theoretically (Hamba, 2001). However, Hamba (2001) has stated that they had to optimize the constants comparing results from i.e. homogenous shear and channel flows with DNS data. Hamba (2001) has found out that the values $\mathrm{C}_{\mathrm{v} 5}$ and $\mathrm{C}_{\mathrm{v} 6}$ should be large enough so that a small value of function $\mathrm{f}$ can ensure realizability. 
Since the model in Eq. 2.46 obtained has second-order nonlinear terms, the non negativeness of the normal stress can easily be shown as a quadratic equation of the mean velocity gradient (Hamba, 2001).

The performance of this new SGS model will be assessed in comparison to the previously applied ones and with various grid resolutions.

The dissipation rate of the energy, $\varepsilon$, is modeled in terms of $\mathrm{k}_{\mathrm{sgs}}$ and the grid filter width, $\Delta$, such as ,

$$
\varepsilon=C_{D} \frac{k_{s g s}^{3 / 2}}{\Delta}
$$

\subsection{Random flow generation (RFG) methodology}

A Random Flow Generation (RFG) approach, originally developed at West Virginia University, provides turbulent initial or inlet flow conditions for LES. In this technique, a divergence free vector field from a sample of Fourier harmonics is synthesized and this allows the generation of a non-homogenous anisotropic flow field representing turbulent velocity fluctuations. The turbulence information needed in RFG is obtained either from measurements or RANS. To generate a realistic flow field, Smirnov et al. (2000) and Shi et al. (2000) proposed a modified version of Kraichnan's technique (Kraichnan, 1970). The relatively simple RFG algorithm involves scaling and simple orthogonal transformation operations applied to a continuous flow-field generated as a 
superposition of harmonic functions. Here, briefly the procedure of the methodology will be described; Given an anisotropic velocity correlation tensor (i.e. from RANS), $r_{i j} \equiv \overline{u_{i} u_{j}}$ of a turbulent flow field, $\left\{\tilde{u}_{i}\left(x_{j}, t\right)\right\}_{i, j=1, . .3}$, first an orthogonal transformation tensor $a_{i j}$ that would diagonalize $r_{i j}$ is found such that

$$
\begin{aligned}
& a_{m i} a_{n j} r_{i j}=\delta_{m n} c_{(n)}^{2} \\
& a_{i k} a_{k j}=\delta_{i j}
\end{aligned}
$$

Then, both $a_{i j}$ and $c_{n}$ become known functions of space. Here, coefficients $c_{n}=\left\{c_{1}, c_{2}, c_{3}\right\}$ play the role of turbulent fluctuating velocities (u', $\left.\mathrm{v}^{\prime}, \mathrm{w}^{\prime}\right)$ in the new coordinate system produced by transformation tensor, $\mathrm{a}_{\mathrm{ij}}$. Further, a transient flow field in a three dimensional domain $\left\{v_{i}\left(x_{j}, t\right)\right\}_{i, j, 1, . .3}$ using the modified method of Kraichan (1970) is generated such that

$$
\begin{aligned}
& v_{i}(\vec{x}, t)=\sqrt{\frac{2}{N}} \sum_{n=1}^{N}\left[p_{i}^{n} \cos \left(\tilde{k}_{j}^{n} \tilde{x}_{j}+\omega_{n} \tilde{t}\right)+q_{i}^{n} \sin \left(\tilde{k}_{j}^{n} \tilde{x}_{j}+\omega_{n} \tilde{t}\right)\right] \\
& \tilde{x}_{j}=\frac{x_{j}}{l}, \tilde{t}=\frac{t}{\tau}, \mathrm{c}=\frac{1}{\tau}, \tilde{k}_{j}^{n}=k_{j}^{n} \frac{c}{c_{(j)}} \\
& p_{i}^{n}=\varepsilon_{i j m} \zeta_{j}^{n} k_{m}^{n}, q_{i}^{n}=\varepsilon_{i j m} \xi_{j}^{n} k_{m}^{n}
\end{aligned}
$$




$$
\zeta_{i}^{n}, \xi_{i}^{n}, \omega_{\mathrm{n}} \in N(0,1), \mathrm{k}_{\mathrm{i}}^{\mathrm{n}} \in N(0,1 / 2)
$$

where $l, \tau$ are the length and time scales of turbulence, $\varepsilon_{\mathrm{ijk}}$ is the permutation tensor and $\mathrm{N}(\mathrm{M}, \sigma)$ is a normal distribution with mean $\mathrm{M}$ and standard deviation $\sigma$. Numbers $k_{j}^{n}, \omega_{\mathrm{n}}$ represent a sample of $\mathrm{n}$ wave number vectors and frequencies of the modeled turbulence spectrum

$$
E(k)=16\left(\frac{2}{\pi}\right)^{1 / 2} k^{4} \exp \left(-2 k^{2}\right)
$$

Finally, a scaling and simple orthogonal transformations to the flow field $v_{i}$ generated is applied to obtain a new-flow field, $\mathrm{u}_{\mathrm{i}}$ as

$$
\begin{aligned}
& w_{i}=c_{(i)} v_{(i)} \\
& u_{i}=a_{i k} w_{k}
\end{aligned}
$$

As a summary, the procedure takes the correlation tensor of the original flow field, $\mathrm{r}_{\mathrm{ij}}$ and the information on length and time scales $(l, \tau)$ as input, where they can be obtained from a steady-state RANS computations or experimental data. Then a timedependent flow field $\mathrm{u}_{\mathrm{i}}(\mathrm{x}, \mathrm{t})$ with correlation functions equal to $\mathrm{r}_{\mathrm{ij}}\left(r_{i j} \equiv \overline{u_{i} u_{j}}\right)$ and 
turbulent length/time scales equal to $l, \tau$. Here, Scaling factors $\mathrm{c}_{\mathrm{i}}$ obtained represent the scales of turbulent fluctuations along each axis, they don't depend on time, whereas vectors $v_{i}$ and $w_{i}$ are time-dependent velocity fluctuations. Spatial and temporal variations of $\mathrm{u}_{\mathrm{i}}$ follow Gaussian distributions with characteristic length and time-scales of $l, \tau$ (Smirnov et al., 2000 and Shi et al., 2000).

This approach was verified using a turbulent channel flow case to check how close the turbulence field from RFG can be compared with measurements or DNS results. It was observed that the DNS results by Kim and Moin (1989) was well reproduced (Shi, 2001).

The features of the generated flow field such as continuity, anisotropy and inhomogenity make the method also well suited for setting initial conditions for LES (Shi et al., 2002).

More details about RFG technique and its validation can be found in Smirnov et al. (2000), Shi et al. (2000) and Smirnov et al. (2001).

\subsection{Verification}

The purpose of model verification is to ascertain that the assumptions made in the solution approach are not violated and the model gives physically reasonable computed results. The four benchmarks, namely, channel flow, flow past square cylinder, 2D mixing layer flow and open channel flow, performed by Shi (2001) are summarized in this section. 
In the turbulent channel flow, relatively higher turbulence intensities compared to experimental results were obtained using the QUICK scheme without SGS model. Much lower turbulence intensities were predicted using SGS eddy viscosity model and the dynamic mixing SGS model (Zang et al., 1993) with CD scheme. However, the predicted fluctuations were in good agreement using Smagorinsky SGS model with $\mathrm{C}_{\mathrm{s}}=0.05$ with CD scheme. Only the location of maximum fluctuations was predicted far from the wall, due to the relatively coarse grid used near the wall (Shi, 2001). The benchmark of flow past a square cylinder, where the body is placed at $\mathrm{x}=0$ (the $\mathrm{x}$-axis is nondimensionalized by $\mathrm{D}$, the length of one side of the square and the $\mathrm{y}$-axis is nondimensionalized by $\mathrm{U}_{\mathrm{o}}$, the inflow velocity) compared with Lyn et al. (1995) has shown that all the different SGS models with different numerical schemes gave relatively good results, however QUICK scheme without any SGS model gave somewhat better results. The features of the vortex shedding were well seen and the inertial range was captured in the energy spectrum at one point in the near wake (2D from the rear edge) (Shi, 2001). A benchmark of the mixing layer flow performed using the Smagorinsky SGS model with CD scheme predicted lower rms velocities in the axial directions than the measurements by Rightley (1995), however the developing Kelvin Helmholtz instabilities were clearly observed (Shi, 2001). To test the capability of the code in representing the free surface as a symmetry boundary, a benchmark of open channel flow was studied and the free surface type velocity profile was able to be obtained (Shi, 2001).

It can be stated that the results obtained from these four benchmarks were in good agreement with either DNS or measurements and the results verified to the feasibility of the current LES approach. 


\section{Chapter 3}

\section{APPLICATION OF EDDY VISCOSITY SGS MODELS}

The subject of this chapter is the application of the numerical schemes and SGS models described in the previous chapters to the calculation of turning ship wake flows. In Section 3.1, the Coriolis force effect on the LES if turning ship wake, for different grid resolutions, the Smagorinky constant effect on TKE, qualitative flow field, comparison of the straight ship to turning ship by comparing the streamwise velocity contours, wake spreading, frequency, spectra, etc. will be covered. In Section 3.2, the assessment of SGS models on open channel flows will be studied such that the results from the effect of the free surface modification in the SGS model, nonlinear one equation model will be

analyzed by profiles obtained for turbulent intensities, $\mathrm{u}^{\prime}, \mathrm{v}^{\prime}, \mathrm{w}^{\prime}$ and the mean flow profile. Application of the FSM to turning ship wake will be discussed. Finally, in Section 3.3, the uncertainty in the present LES will be introduced with several calculations. 


\subsection{Application of Smagorinsky model to the wake of a Turning Ship}

As a first step for the present study, a standard Smagorinsky model that forms the basis for the advanced models has been applied to investigate the influence of the Coriolis and centrifugal forces on turbulence generation on the ship wake flow field.

The focus of this study is the wake behind the Navy DDG51 surface ship, which is approximately $154 \mathrm{~m}$ long and $20 \mathrm{~m}$ wide, cruising on a circular track (see Figure 3.1 ). The average speed of the ship is assumed to be around 20 knots. The ship model data used for this simulation was taken from the data of DTMB 5415, a towing tank model representing a modern naval combatant, DDG51 (Stern et al., 2000). As stated in Shi (2001) and Yavuz et al. (2002), the ship hull is excluded from the LES calculations due to computational expenses and the computations were started from a plane aft of the ship. This is accomplished using the RFG technique, originally developed at West Virginia University, which calls for a time averaged flow field at the inlet data plane. Reynolds Averaged Navier Stokes (RANS) calculations (Hyman, 2001) (steady state RANS calculations around the ship hull) are used to provide the RFG procedure with the information needed on the inflow boundary (initial data plane) located $0.5 \mathrm{~L}$ after the body in the wake. In other words, the pseudo random flow field generated by the RFG technique is added to the mean flow of the RANS simulations in order to provide the boundary condition at the inlet plane. The further development of the wake flow is calculated via LES (non steady LES of the wake). LES of high Reynolds number flows with complicated geometries are enabled by this combined approach (Smirnov et al., 2000). Therefore, the effect of the ship body on the flow field is embedded in the mean flow prescribed at the inflow plane. However, this technique, also known as the Initial 
Data Plane (IDP) approach (Hyman, 1998, Paterson et al., 1996) can introduce considerable errors (Hyman, 1998), as a matter of fact that the body generating the wake is not included in the non-steady LES simulations. It should be noted that it is possible to predict turbulence via LES technique by starting from a mean flow obtained from RANS. However, it takes a very long time for a turbulent flow to develop spatially and temporally without any initial perturbation. Also for some engineering applications, it may be too expensive in terms of computer resources and programming effort.

\subsubsection{Conditions and Grids}

As stated above, the ship model DTMB 5415 is a $5.72 \mathrm{~m}$ long model of the Navy DDG51 surface ship (Stern et al., 2000). A computational domain of $1.75 \times 0.15 \times 1.0$ (given in non dimensional units in ship lengths and it starts from $\mathrm{x} / \mathrm{L}=1.50$, where $\mathrm{x} / \mathrm{L}=1$ is the end of the ship model) and a grid of $66 \times 33 \times 66$ (Coarse Grid) (Case-1) in $\times$ (axial), $\mathrm{y}$ (vertical), z (transverse) directions has been used to represent the near wake region. This grid configuration has been selected to quickly assess the effect of the Coriolis and centrifugal forces on the turbulence characteristics. It should be noted that a thorough study was not conducted as to whether the side boundary is far enough away from the wake. In the IDP plane, the RFG method is used in conjunction with the RANS calculations (Hyman, 2001) and the ship's stern is at $(0.5,0,-3.0)$ in $x^{-}, y^{-}, z-$ directions respectively. The core region of the numerical grid and the geometry of the ship model are illustrated in Figure 3.2 (a) \&(c). The ship turns with a dimensionless angular velocity of $1 / 3$ (Figure 3.2 (b)) with a radius of curvature corresponding to three dimensionless 
ship lengths, which will result in a dimensionless ship velocity of 1 . Only a $30^{\circ}$ turn was investigated. Two Reynolds numbers have been used in the simulations. One is based on the real ship length, i.e. $1.5 \times 10^{9}$ stated as Case- 1 and the other based on the model ship length, i.e. $1.0 \times 10^{7}$ stated as Case-2. Here, Reynolds number similarity is assumed, which is attained by changing the laminar viscosity of the fluid in the simulations. The coordinate system used is with respect to an observer on the ship. The Smagorinsky constant used in the eddy viscosity relation (Eqn. 1.3) is calculated to be 0.042 from scaling with the non-turning simulations (Shi, 2001). Moreover, a simulation with relatively fine grid in the axial direction has been conducted to investigate the grid

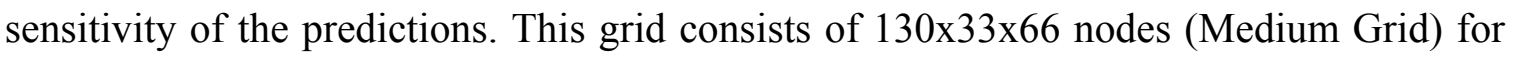
Case -1 in $\mathrm{x}, \mathrm{y}$, and $\mathrm{z}$ directions, respectively. Non-uniform grids were used in this study in both $\mathrm{x}$ and $\mathrm{z}$ directions with the expansion ratio not exceeding 1.03 . The length scale and time scale used in RFG are calculated from $\tau=\mathrm{k} / \varepsilon$ and $1=0.09 \mathrm{k}^{1.5} / \varepsilon$, non dimensionalized by free stream velocity and ship length. A comparison of the results from using Smagorinsky constants, 0.065 (selected to be the constant for the non-turning ship simulation) and 0.042 has been presented for the relatively fine simulations. Then,

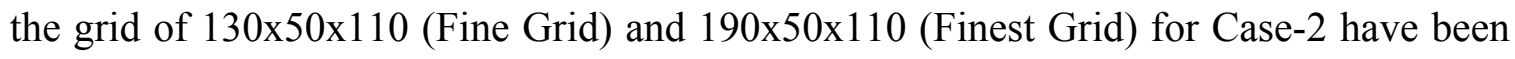
used to analyze the physics of the wake behind a turning ship. Finally, to investigate the effect of the free surface, another study has been conducted with fine grids for the model ship simulation. The time step is 0.001 for all grid resolutions. On an Intel Pentium 4 $3 \mathrm{GHz}$ machine, CPU-time and memory requirements were approximately 226 hours for one flow through time (time required for the flow to pass in the calculation domain, 
$\mathrm{x}=1.75 \mathrm{~L})$ and 491 megabyte respectively, for the fine grid simulations with the standard Smagorinsky model.

On the other hand, Shi (2001) and Shi et al. (2002) applied LES on the wake flow of the ship model DTMB 5512 (Longo et al., 1993, Gui et al., 1999, Stern and Wilson, 2000). A $3.048 \mathrm{~m}$ long unpropelled model of a modern U.S. Navy fleet ship, ArleighBurke class destroyer, DDG51, with a Reynolds number of $4.65 \times 10^{6}$ cruising on a straight track has been investigated. The computational domain was $1.5 \times 0.3 \times 0.6$ (given in non dimensional units in ship length) in $\mathrm{x}-, \mathrm{y}-, \mathrm{z}$-directions, respectively, with a grid size of $162 \times 50 \times 66$ and $322 \times 50 \times 66$. Non-uniform grid spacing with stretching smaller than 1.03 was used in both $\mathrm{x}$ and $\mathrm{y}$ directions.

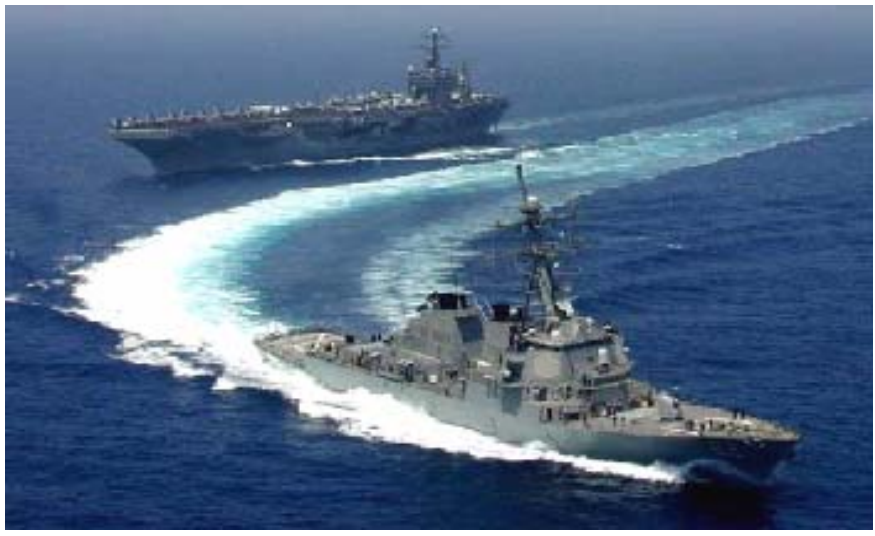

Figure 3.1. Arleigh-Burke class destroyer (DDG51) 

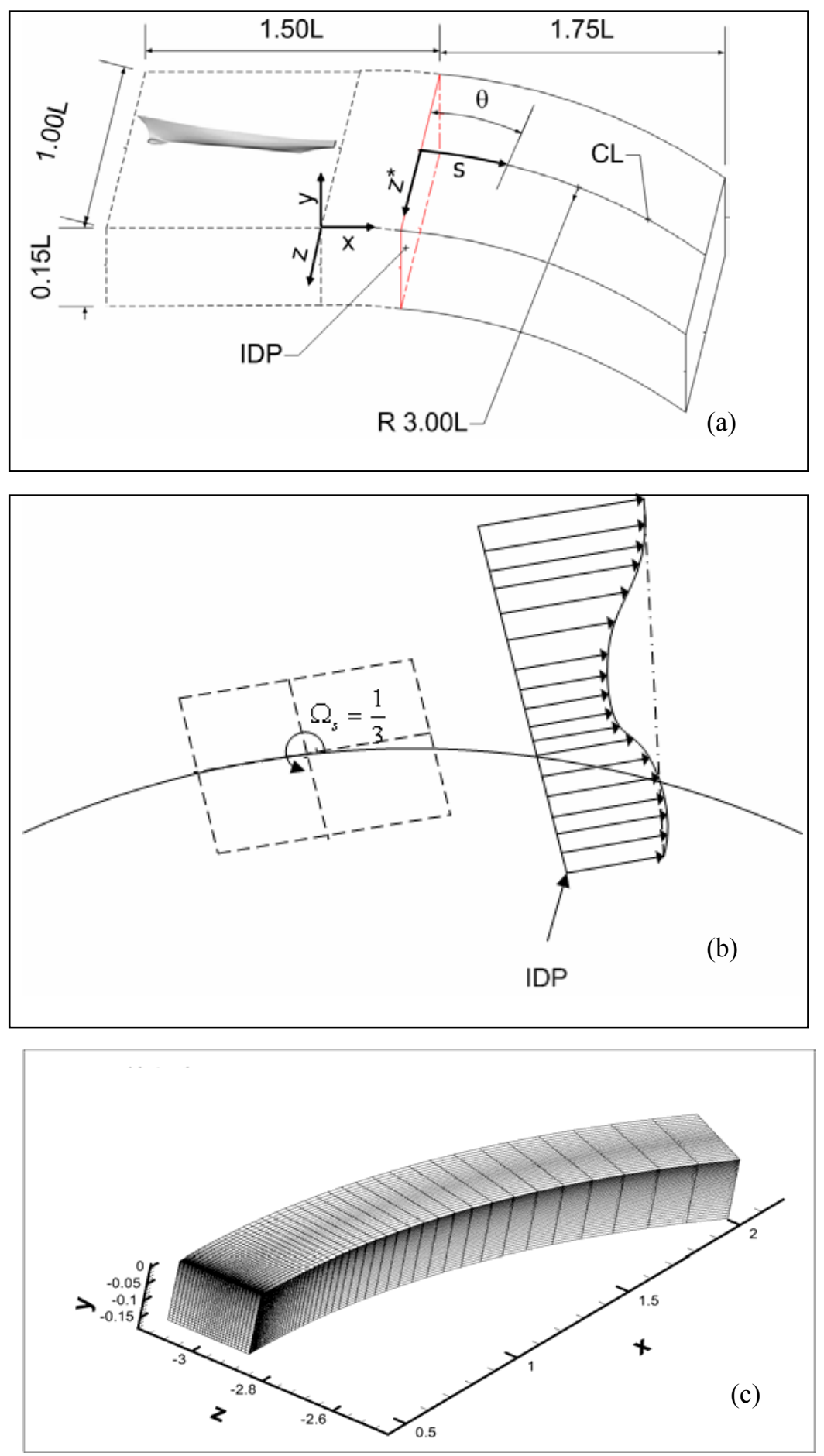

Figure 3.2. Turning ship wake: a) The geometry b) velocity profile specified at the IDP (top view) c) The coordinate space system and numerical grid (Only the core region is shown, distances are non-dimensionalized with ship length, L) 


\subsubsection{Boundary Conditions}

For all grid simulations, the velocity components at inflow boundary plane are set equal to those calculated via RANS simulations (Hyman, 2001) plus the fluctuating velocity component obtained from the RFG technique (see Figure 3.2. (b)). The outflow boundary is assumed to be a free gradient boundary. At the top and bottom boundary surfaces a symmetry boundary condition is applied. At the free surface (i.e. the top boundary), a slip is allowed in $\mathrm{x}$ and $\mathrm{z}$ directions but the velocity component normal to the free surface is set to zero (as such the free surface is approximated as a moving flat plane; where the free surface is completely flat). The boundaries in the transverse direction are treated as stream surfaces, where the tangential velocities are specified using the turning ship velocity as a base and by accounting for the turning of the ship via a rigid body motion, such as,

$$
\bar{u}_{t}=\Omega_{s} *\left(\frac{R^{*}-z^{*}}{L}\right)
$$

$\Omega_{\mathrm{s}}=$ the angular velocity of the system rotation due to the turning ship

$\mathrm{R}^{*}=$ the radius of the curvature, $\mathrm{R}^{*}=3.00 \mathrm{~L}$

$\mathrm{z}^{*}=$ the distance measured from the centerline in the positive $\mathrm{z}$ direction 


\subsubsection{Results and Discussions}

Figure 3.3 shows the instantaneous vertical velocity contours with Coriolis force (Case-1) at different y-z planes in the turning ship wake. This figure has been included to help the reader to visualize the cross sectional planes taken at different angles $\left(5^{\circ}, 10^{\circ}\right.$, $15^{\circ}, 20^{\circ}, 25^{\circ}, 30^{\circ}$ ). Figure 3.4 shows the radial velocity profiles at two different $y-z$ planes $\left(5^{\circ}, 10^{\circ}\right)$ for fine grid in order to check the boundary conditions specified in the transverse direction. The figure proves that the LES code treats the boundaries in the transverse direction as stream surfaces. The unsteady velocity fluctuations are compared for cases with and without Coriolis force (Case-1) in Figures 3.5 to 3.7. The figures show the streamwise velocity components versus nondimensional time at an angle of 10 degrees measured from the inlet plane z. From approximate calculations, the resolved frequency of the velocity fluctuations is obtained to be around $4.7 \mathrm{~Hz}$ in all three directions with the Coriolis force and $3.5 \mathrm{~Hz}$ without the Coriolis force. This indicates that the Coriolis force tends to increase turbulence activity. In order to compare the results of the turning and non turning ship wake cases, a scaling has been done such that, from Equation 2.36, $\left.\left.\left(C_{s} \Delta^{2}\right)\right|_{\text {nonturn }} \cong\left(C_{s} \Delta^{2}\right)\right|_{\text {turn }}$. From Shi's (2001) non turning ship wake calculations, $\mathrm{Cs}$ is set to be 0.065 . Using the above scaling, $\mathrm{C}_{\mathrm{s}}$ of 0.042 has been calculated and used for the medium grid simulations. For fine and finest grid simulations, similar logic has been carried out in order to obtain $\mathrm{C}_{\mathrm{s}}$ values in the Smagorinsky model. For the medium grid, the resolved frequency is around $9 \mathrm{~Hz}$ for $\mathrm{C}_{\mathrm{s}}=0.042$ and $7 \mathrm{~Hz}$ for $\mathrm{C}_{\mathrm{s}}=0.065$. The frequencies are approximately calculated from the temporal history curves

of all three directions, such that one over the difference of the two crest points divided by 
the time difference in those crest points gives the approximate frequency. If the Smagorinsky constant is smaller, the frequency obtained is higher, which implies that more energetic turbulent fluctuations are captured. This is an indication that higher frequencies and smaller turbulence scales are captured with the relatively fine grid for both values of the Smagorinsky constant. Hence, physical intuition and simulations give similar conclusions.

Figures 3.8-3.10 present the velocity vectors at vertical cross-sections at an angle of $5^{\circ}$ with the z-axis Note that $\mathrm{R}$ is taken to be minus to be consistent with the minus $\mathrm{z}$ direction, that is calculated from $R=\sqrt{(x-0.5)^{2}+y^{2}}$. The coarse grid case in Figure 3.8 with the Coriolis force shows better-defined turbulent structures or eddies than the case without the Coriolis force in Figure 3.9, which seems to be more diffusive. Here, one could argue that the Coriolis force helps to maintain the turbulent structures of the flow. Moreover, the medium grid predictions with the Coriolis force presented in Figure 3.10 indicate even a lesser diffusion (or smearing) for both values of Smagorinsky constant. Here, it can be seen that the vortical structures are not penetrating as deeply as in the coarse grid case. Experimental observations of Matsubara and Alfredsson (1998) have shown that the Coriolis force may give rise to instabilities in the form of longitudinal vortices which supports the above assertions.

The velocity vectors on vertical cross-sections at an angle of $25^{\circ}$ with the z-axis are shown in Figures 3.11-3.13. Although, similar structures in both near the inlet data plane and near the outlet are observed, the intensity of the velocity fluctuations diminish as the outflow plane is approached due to grid expansion. Moreover, it is seen that away from the wake centerline, the strength of the structures has already died in both cases. In 


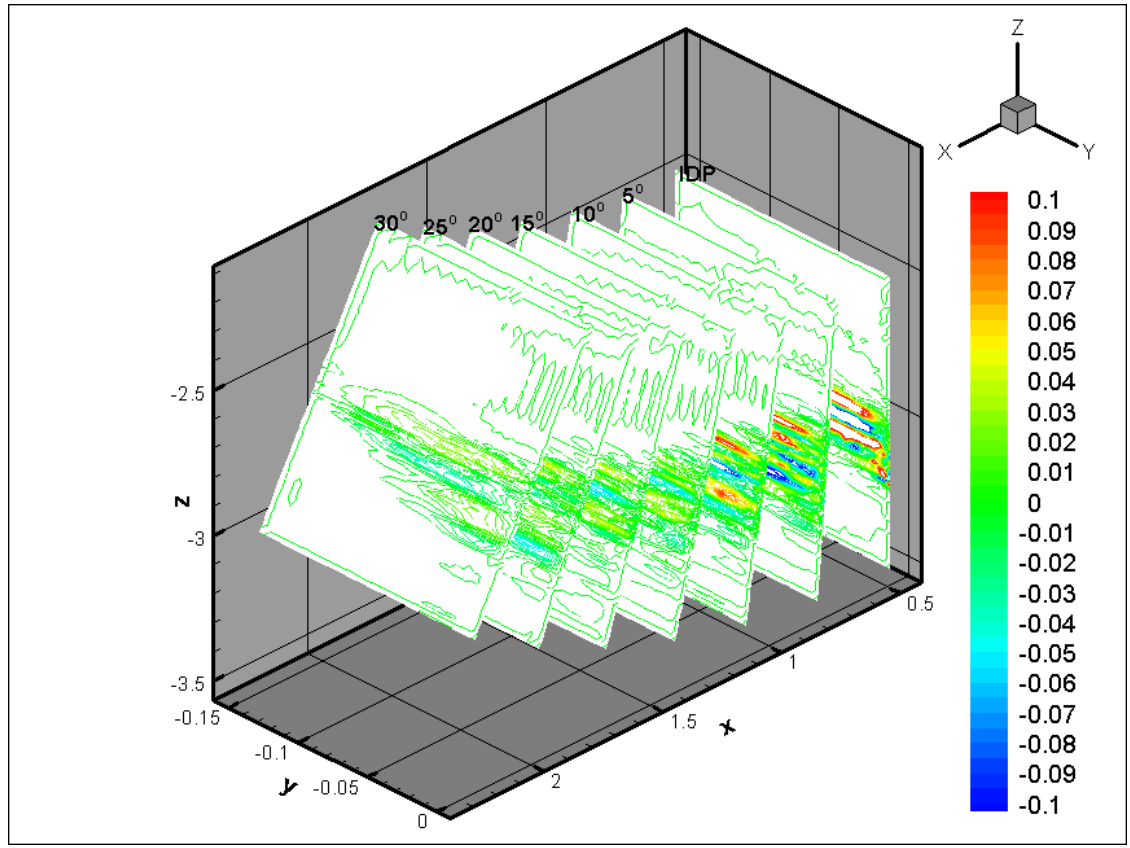

Figure 3.3. Typical instantaneous vertical velocity contours w Coriolis force on different y-z plane in the turning ship wake (coarse grid)

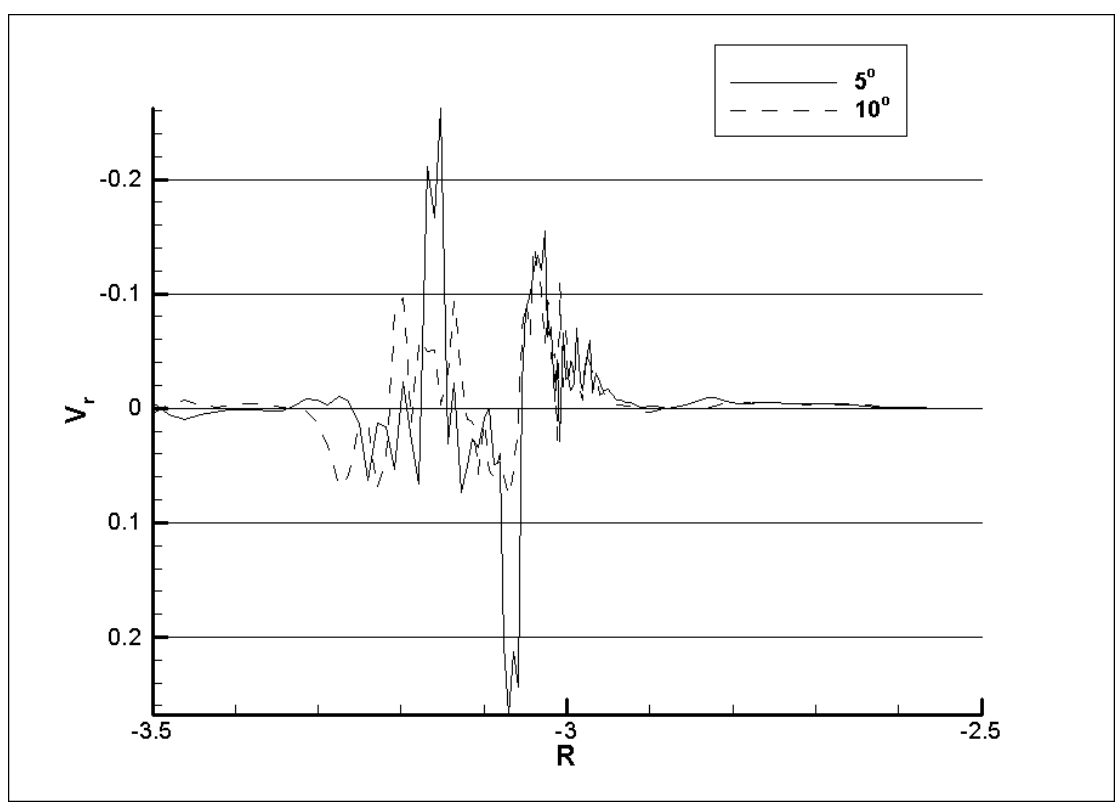

Figure 3.4. Radial velocity profiles on different y-z planes in the turning ship wake (fine grid) 

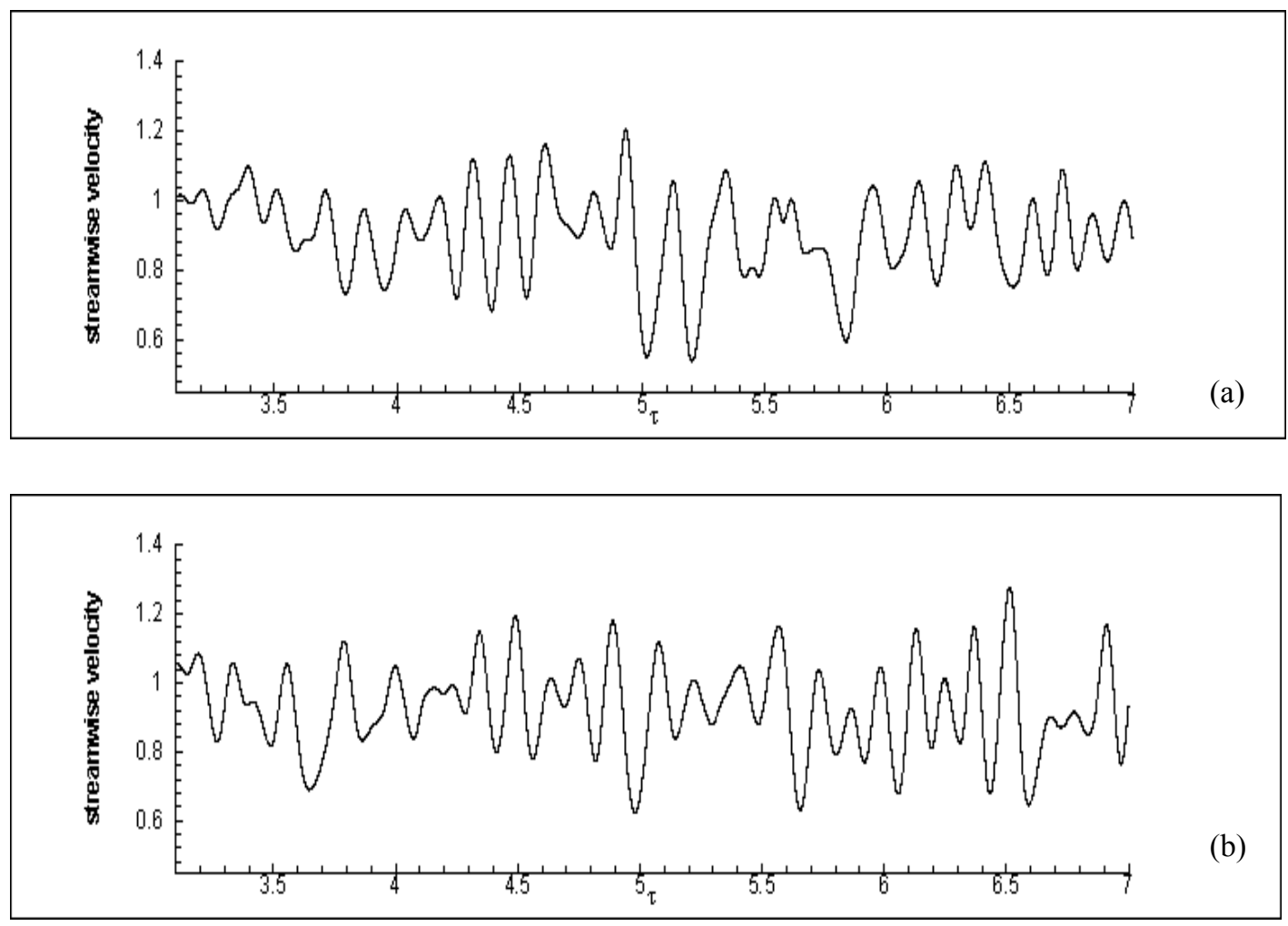

Figure 3.5. Temporal history of streamwise velocity components at $10^{\circ}$ with the $\mathrm{z}$ axis, and $\mathrm{x}=1.02, \mathrm{y}=-0.002$ (coarse grid), a) w Coriolis force $\mathrm{b}$ ) w/o Coriolis force

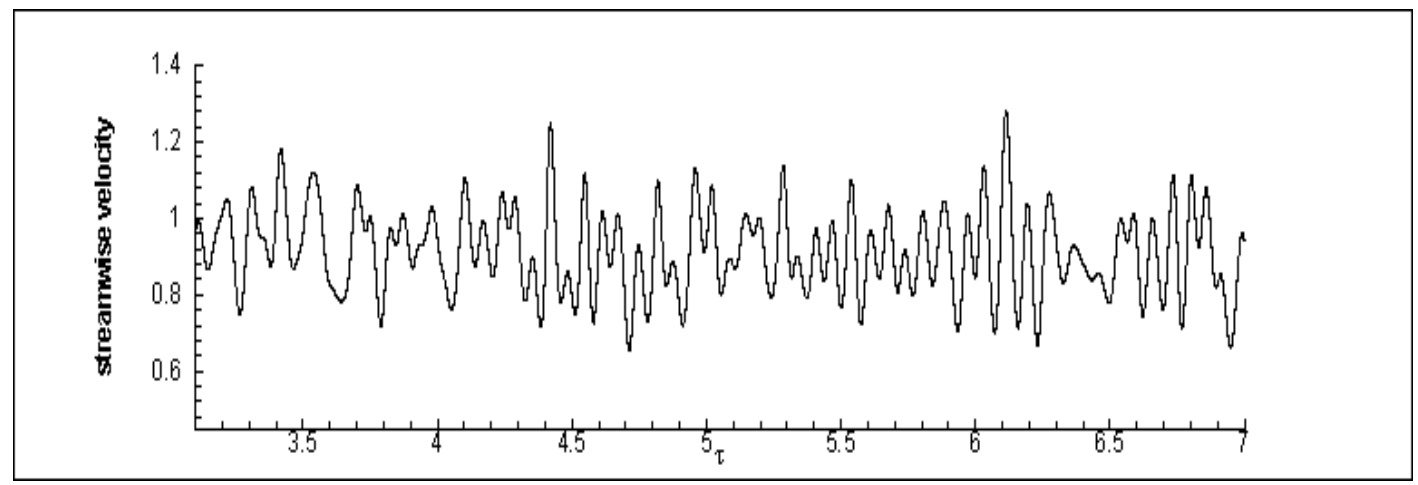

Figure 3.6. Temporal history of streamwise velocity components at $10^{\circ}$ with the $\mathrm{z}$ axis, and $\mathrm{x}=1.02, \mathrm{y}=-0.002$ (medium grid), $\mathbf{C}_{\mathrm{s}}=\mathbf{0 . 0 4 2}$ 


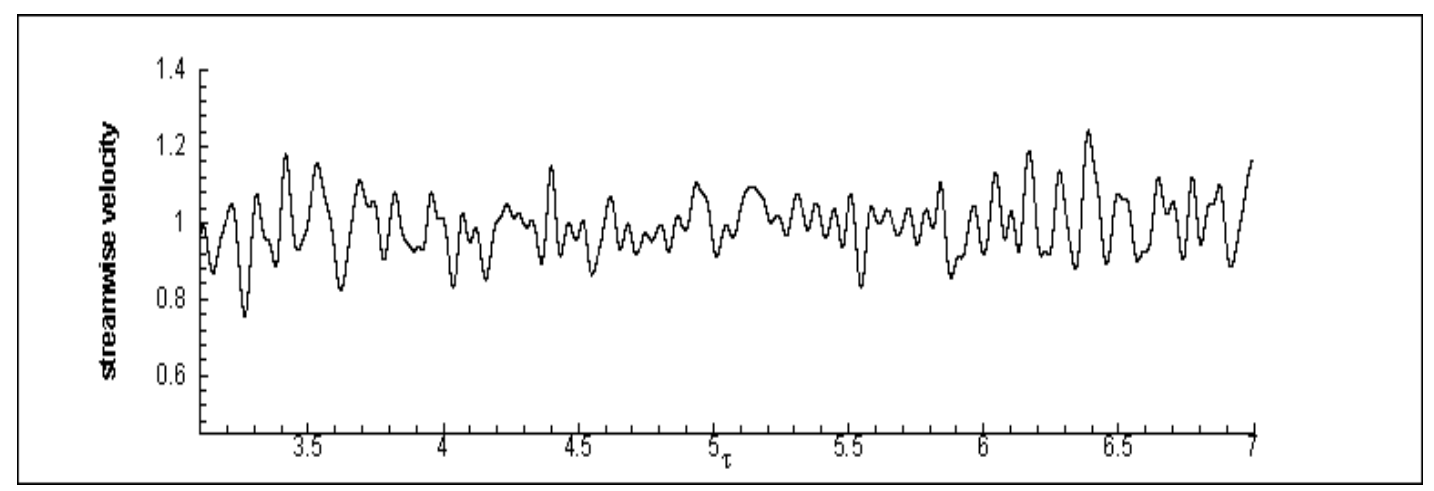

Figure 3.7. Temporal history of streamwise velocity components at $10^{\circ}$ with the $\mathrm{z}$ axis, and $\mathrm{x}=1.02, \mathrm{y}=-0.002$ (medium grid), $\mathbf{C}_{\mathbf{s}}=\mathbf{0 . 0 6 5}$

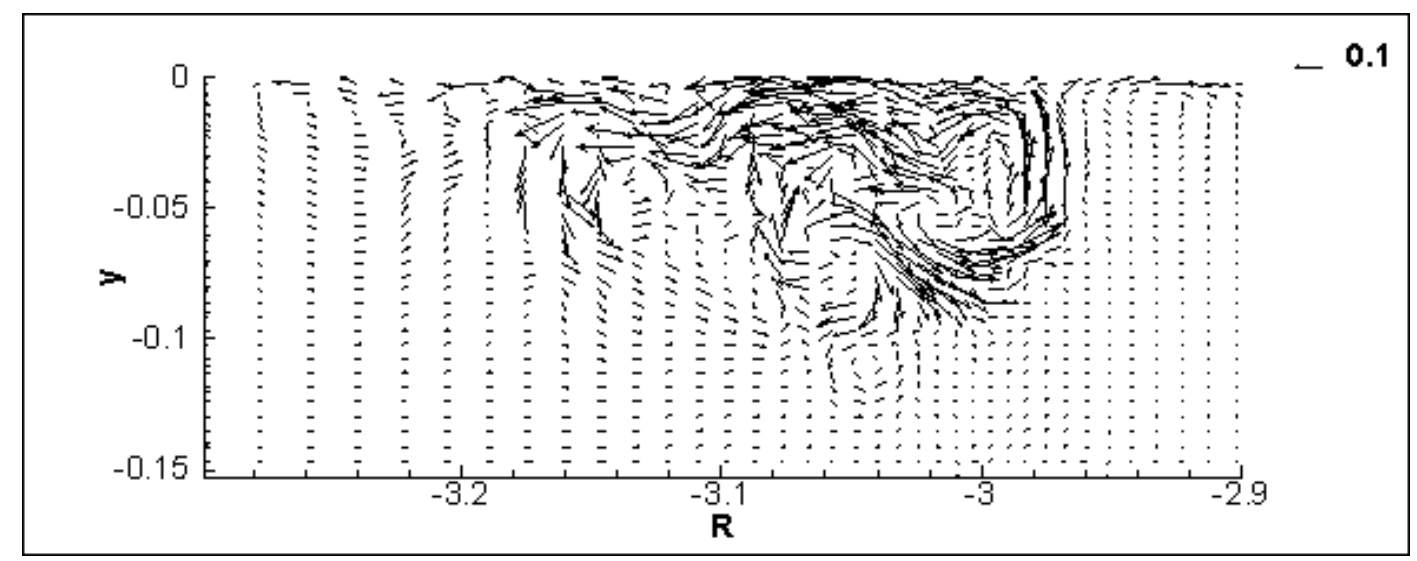

Figure 3.8. The velocity vectors on a vertical plane at an angle of $5^{\circ}$ with the $\mathrm{z}$-axis with Coriolis force (coarse grid) 


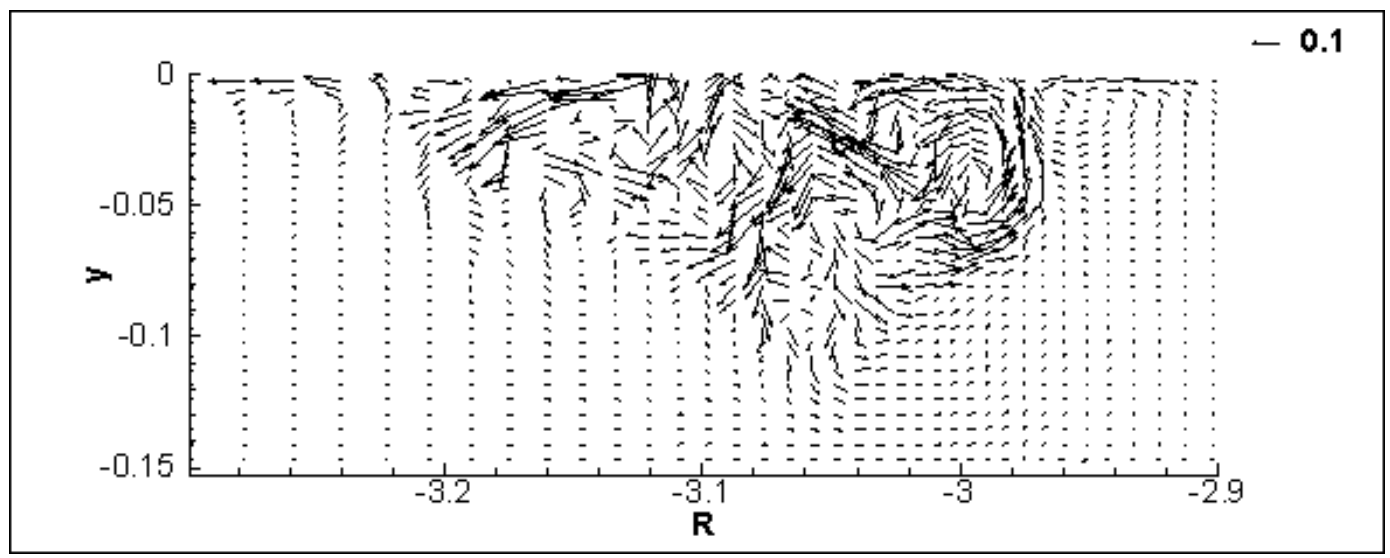

Figure 3.9. The velocity vectors on a vertical plane at an angle of $5^{\circ}$ with the z-axis without Coriolis force (coarse grid)
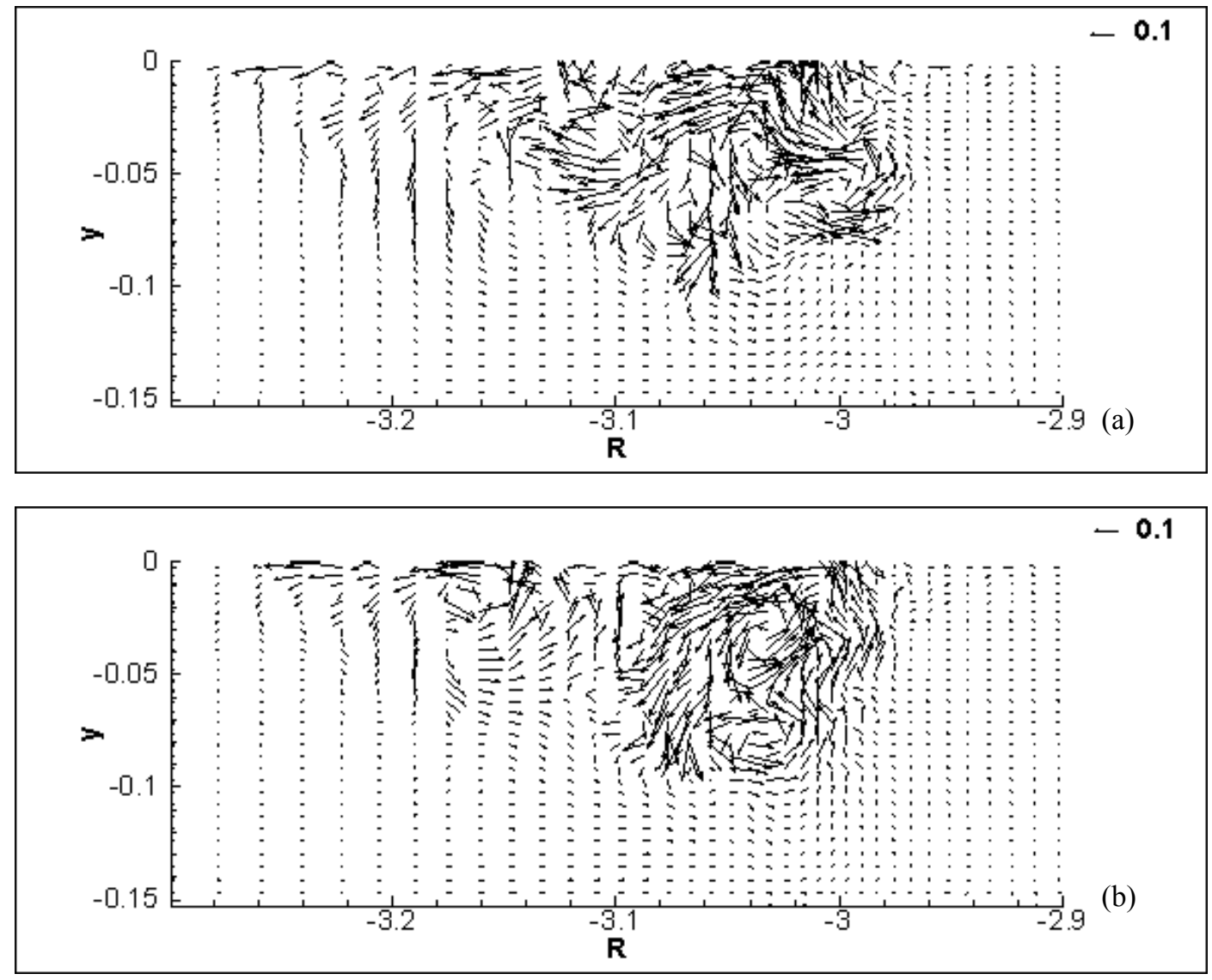

Figure 3.10 . The velocity vectors on a vertical plane at an angle of $5^{\circ}$ with the $\mathrm{z}$-axis (medium grid) a) $\mathrm{C}_{\mathrm{s}}=\mathbf{0 . 0 4 2}$ b) $\mathrm{C}_{\mathrm{s}}=\mathbf{0 . 0 6 5}$ 
the far downstream portion of the calculation domain, larger turbulent structures are seen to be merging with the smaller structures. Still, a significant level of vorticity can be captured in both cases. Overall, it is seen that one large vortex, presumably the one originating from the ship hull persists without significant dissipation.

A comparison of the resolved turbulence kinetic energy is shown in Figure 3.14. In calculating the kinetic energy values, the samples were taken to be 10000 time steps from 2 flow through time data ( $=35000$ time steps). Here, it is observed that due to the coarse grid towards the end of the calculation domain, vorticity (hence turbulence) decays rapidly, however the medium grid predictions have a very gradual decay of turbulent kinetic energy. For the coarse grid simulations, turbulence decays more rapidly due to the numerical dissipation. The kinetic energy values obtained from the case with Coriolis force is slightly higher than that of the case without Coriolis force, as expected. As seen from the figure, the adjustment of the Smagorinsky constant is necessary, as the kinetic energy values obtained with $\mathrm{C}_{\mathrm{s}}=0.042$ are almost on the same level as the ones obtained from the non-turning ship simulations(Shi, 2001). The turbulence intensity specified at the inlet was observed to decay rapidly, which was mainly due to the nature of the coarse grid used. For this reason a medium grid simulation has been performed. This improved the turbulent kinetic energy prediction and more detailed turbulence structures were captured.

Similar conclusions were also drawn from the vorticity contours. Calculations showed that the highest and lowest vorticity magnitudes are more pronounced in the case with the Coriolis force, whereas the case without the Coriolis force shows a more 


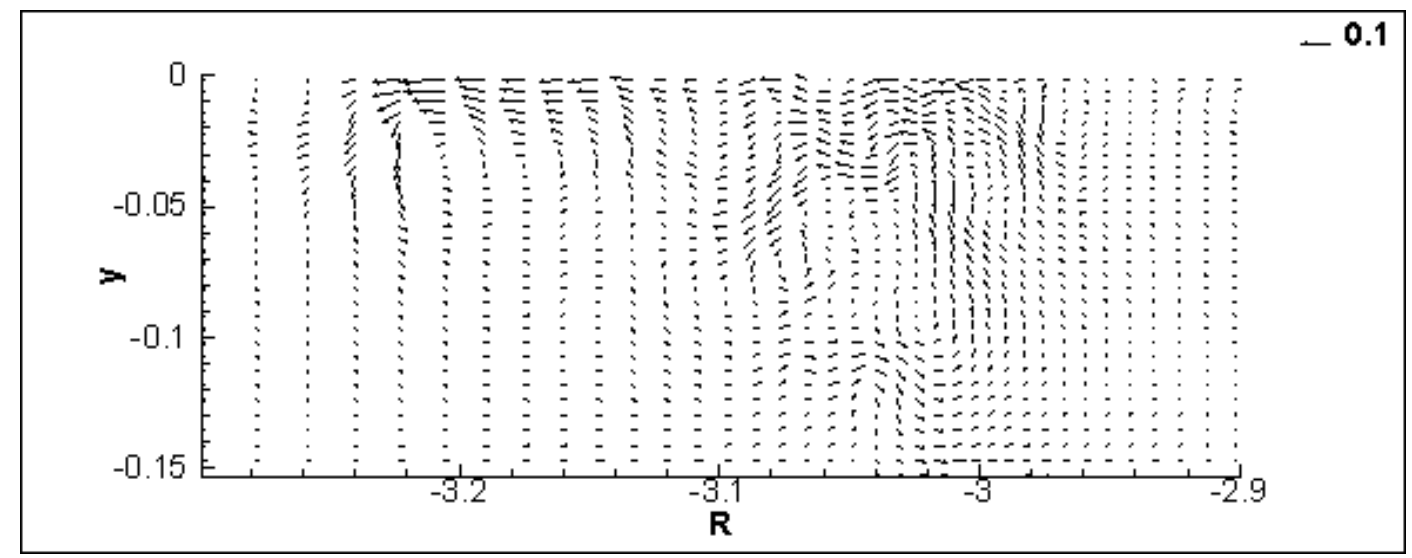

Figure 3.11. The velocity vectors on a vertical plane at an angle of $25^{\circ}$ with the $\mathrm{z}$-axis with Coriolis force (coarse grid)

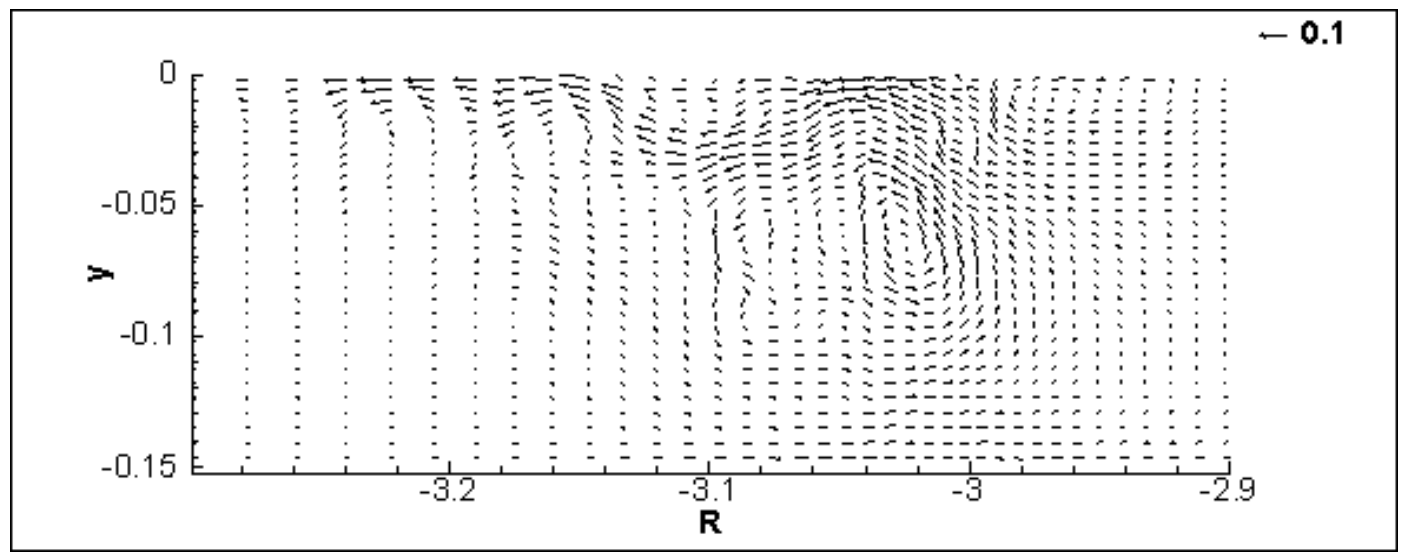

Figure 3.12 . The velocity vectors on a vertical plane at an angle of $25^{\circ}$ with the $\mathrm{z}$-axis without Coriolis force (coarse grid) 

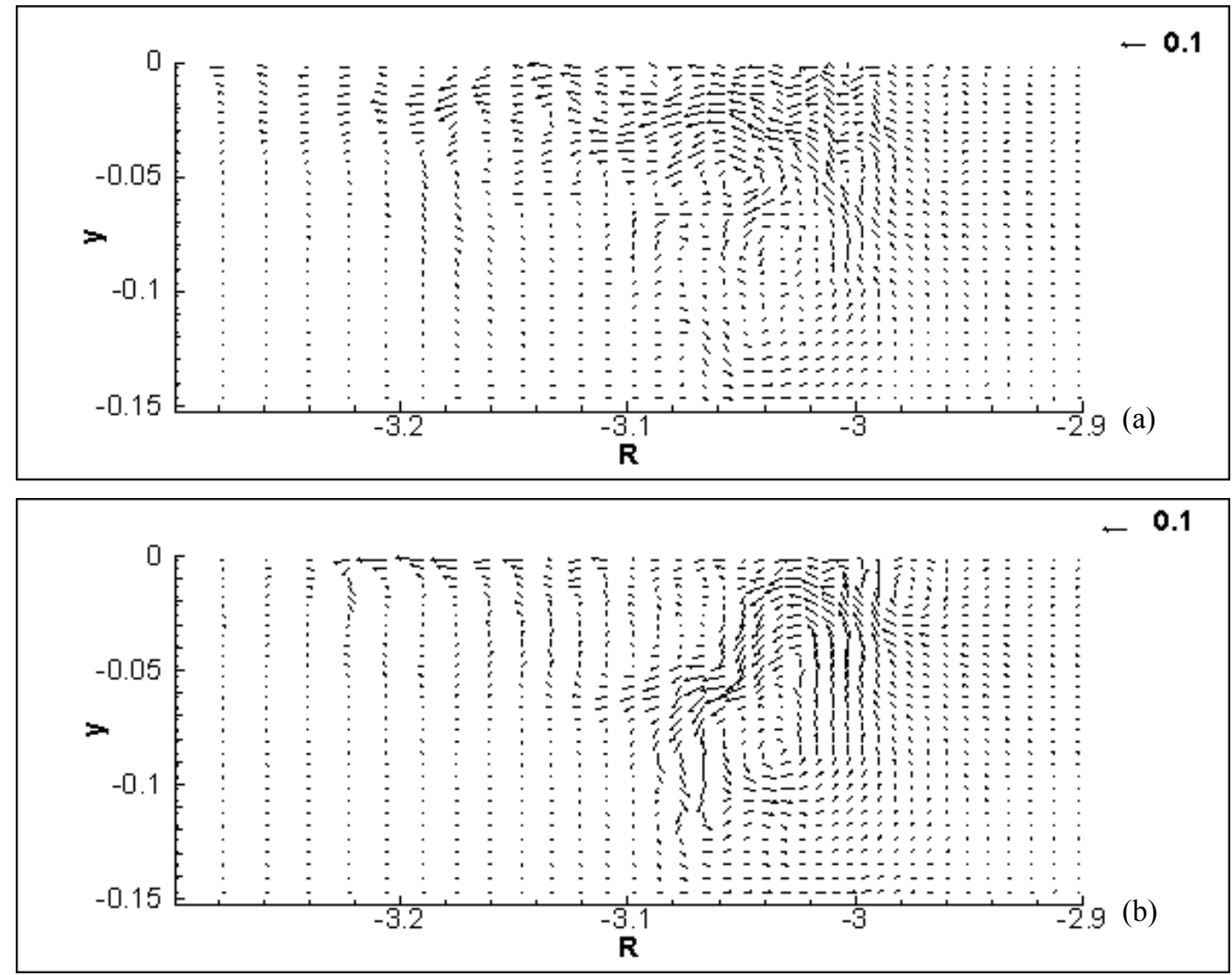

Figure 3.13 . The velocity vectors on a vertical plane at an angle of $25^{\circ}$ with the $\mathrm{z}$-axis (medium grid) a) $\mathrm{C}_{\mathrm{s}}=\mathbf{0 . 0 4 2}$ b) $\mathrm{C}_{\mathrm{s}}=\mathbf{0 . 0 6 5}$ 


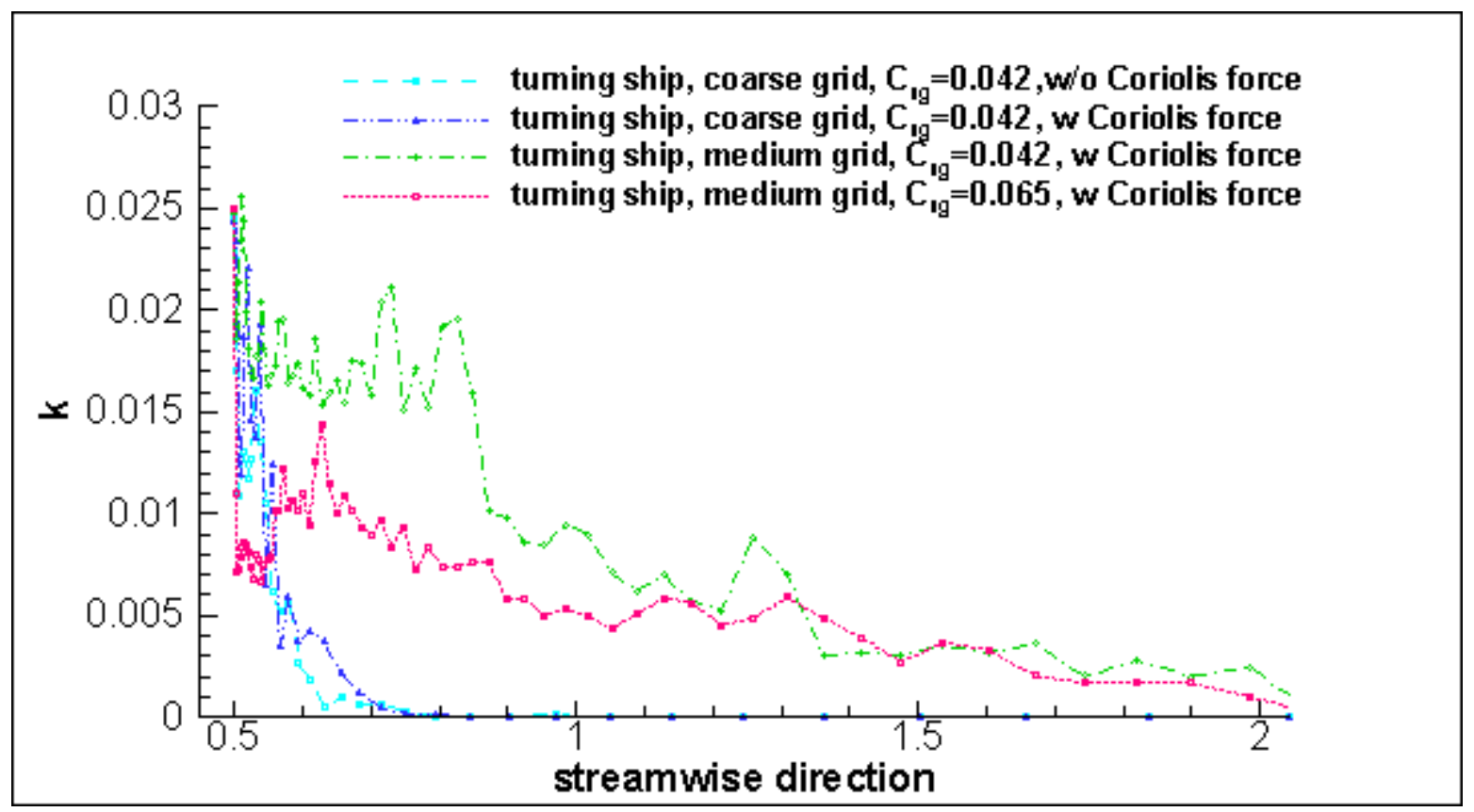

Figure 3.14. The comparison of the resolved turbulence kinetic energy for ship cruising on a circular track 
diffused or smeared picture. The vorticity results again indicate that the Coriolis force keeps the vorticity concentrated in the flow-field.

The goal of this preliminary study was to assess the effects of the Coriolis and centrifugal forces on the vortical structures or turbulence characteristics of the flow in the wake of a turning ship using the large eddy simulation technique. The eddies resolved by LES have been observed to be more energetic and less diffusive when the Coriolis force was included. It seems as if this force supplies energy to the large turbulent structures and thus enhances anisotropy. The vorticity contours show a non-symmetric wake development with significant stretching in the radial direction away from the center of rotation. This is also seen from the velocity vectors comparison of non-turning and turning ship studies in Figures $3.15 \&$ 3.16. As these figures show the non-turning ship case has a symmetric wake with respect to the wake centerline, whereas in the turning ship case, there is a significant flow present in the radial direction that is believed to be caused in part by the centrifugal force, arising from turning of the ship. Here, the simulation of the model ship with grids of 130x50x110 and 190x50x110 has been studied to compare the results with the ship model of the non-turning ship wake.

A study of the effect of Reynolds number for Case-2 and Case-1 using 190x50x110 grids is shown in Figure 3.17. This comparison can be done since all the values are nondimensionalized with respect to their ship velocities, either real ship or the ship model. The velocity vectors are at the same location, $\mathrm{x} / \mathrm{L}=0.65$ and standard Smagorinsky model has been used. When the Reynolds number is low, the turbulence structures are observed to be more visible, well defined and more diffusive. However for 

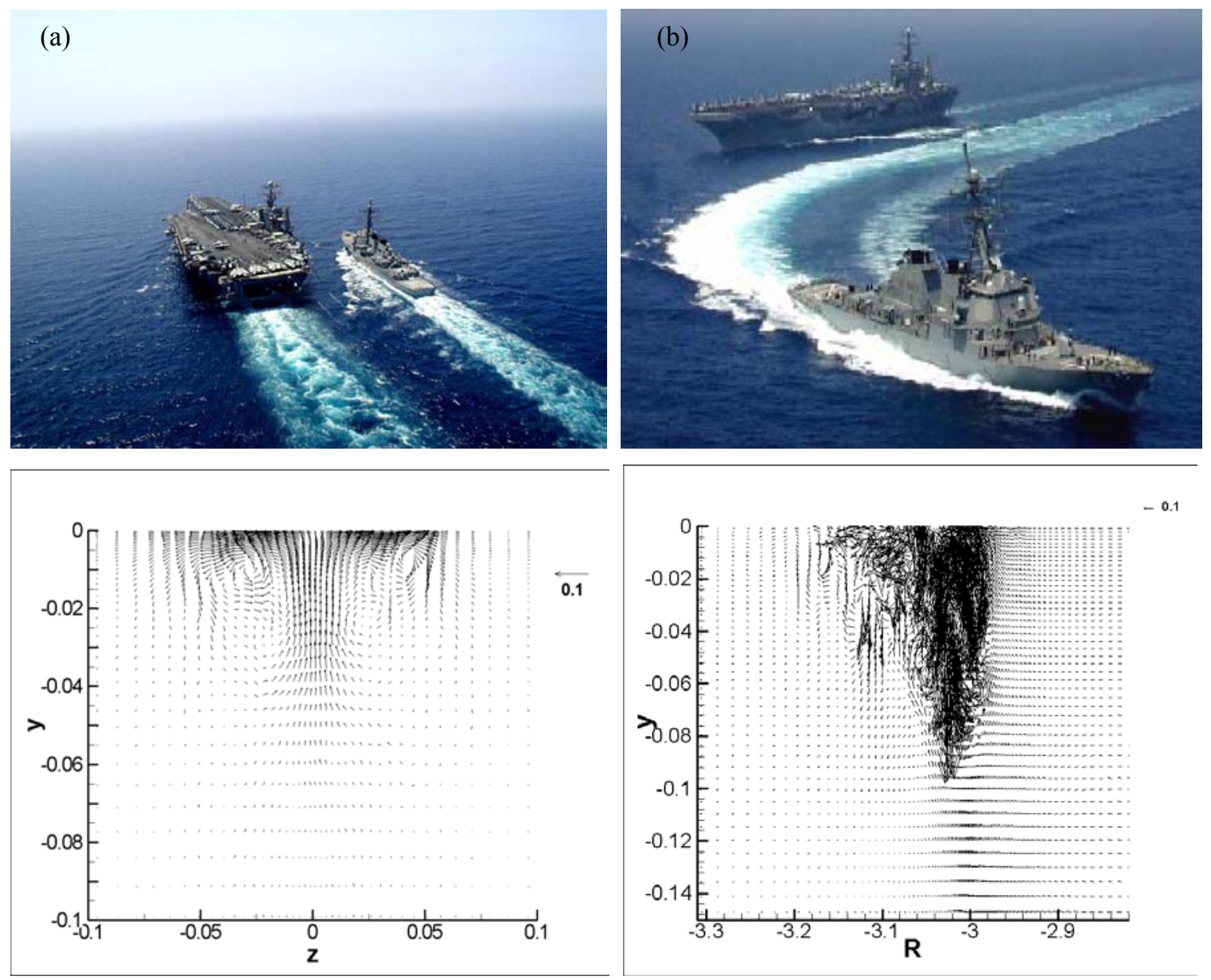

Figure 3.15. Velocity vectors at $\mathrm{x} / \mathrm{L}=0.2$ : a) Non-turning ship (Shi et al. 2001) (fine grid)

b) Turning ship (fine grid) 

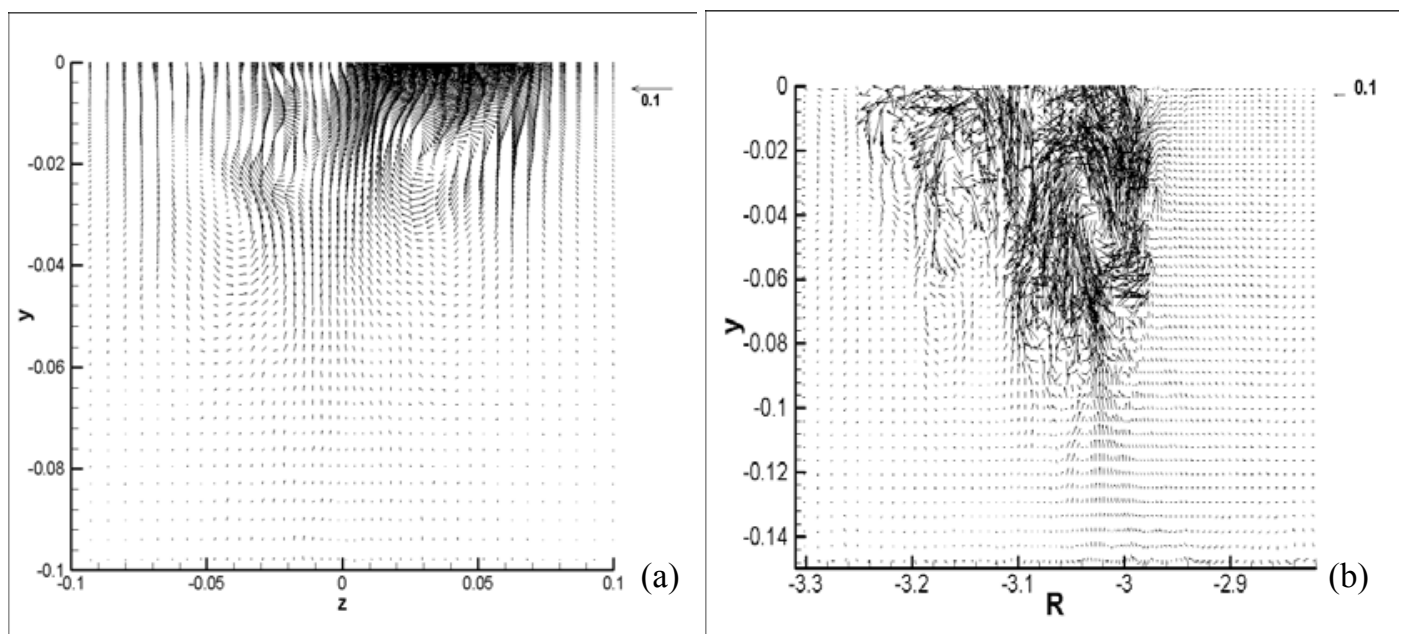

Figure 3.16. Velocity vectors at $\mathrm{x} / \mathrm{L}=0.65$ a) Non-turning ship (Shi et al. 2001) (finest grid) b) Turning ship (finest grid)
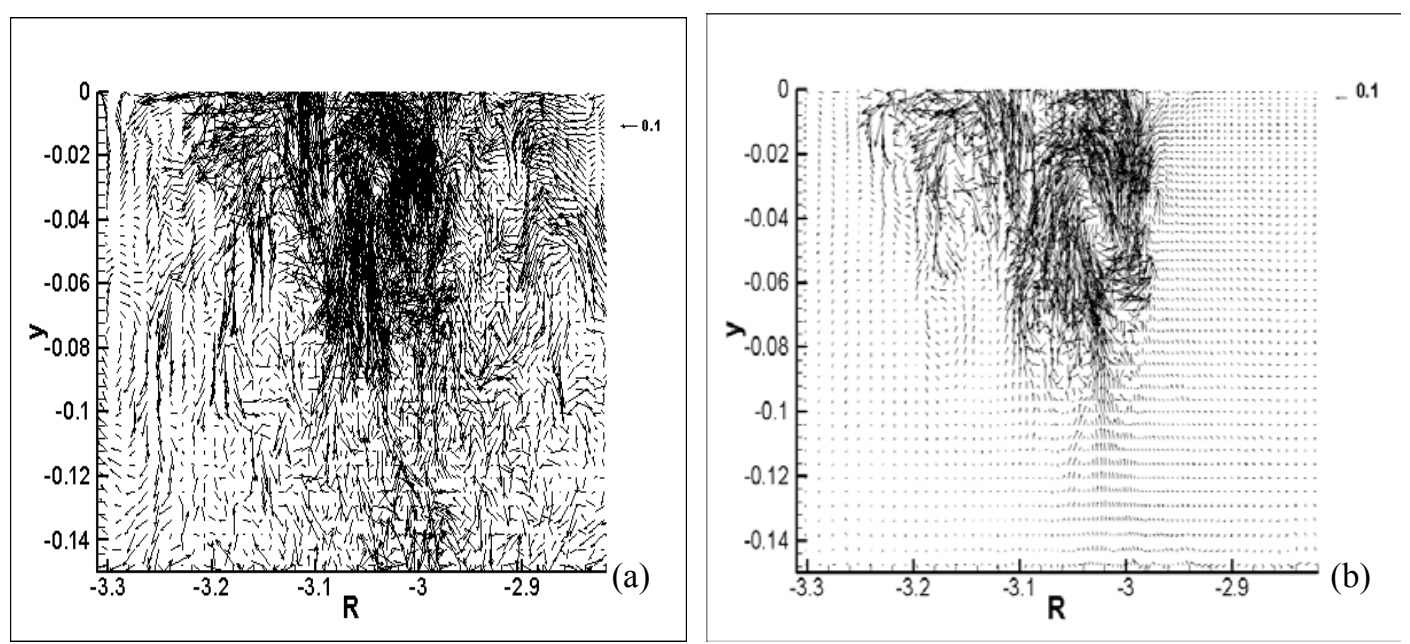

Figure 3.17. Velocity vectors at $\mathrm{x} / \mathrm{L}=0.65$ for turning ship wake a) Case- $1 ; \mathrm{Re}=1.5 \times 10^{9}$ : DDG51 (finest grid) b) Case-2; $\mathrm{Re}=1.0 \times 10^{7}$ : DTMB 5415 (finest grid) 
high Reynolds number flows, there are certain unorganized structures seen in the flow field and the flow structures seem to be less diffused. As the wake develops, the flow structures disappear rapidly for Case-1; however, for Case-2, the flow structures are observed to be still well defined. The unsteady velocity fluctuations are also compared for Case-1 and Case-2 in Figures 3.18. The resolved frequency of the velocity fluctuations is estimated to be around $40 \mathrm{~Hz}$ in all three directions for Case- 1 and $30 \mathrm{~Hz}$ for Case-2. Here, $30 \mathrm{~Hz}$ and $40 \mathrm{~Hz}$ seem to correspond to large bilge vortex passage. If the Re is higher, the frequency obtained is higher, which is an indication that smaller turbulent structures are captured with high Reynolds number. The frequency can also be computed from the roughly eddy turnover, which is $\mathrm{T}=$ largest eddy size/ship velocity where at $10^{\circ}$, the largest eddy size is approximately 0.04 from Figure 3.23 (b) and the ship velocity is 1 , therefore $\mathrm{T}=0.04 / 1=0.04, \mathrm{f}=1 / \mathrm{T}=25 \mathrm{~Hz}$, which is close to $30 \mathrm{~Hz}$.

Although the classical Smagorinsky SGS model is not that suitable for complex flows as it uses a constant eddy viscosity coefficient for the entire domain, this study has shown that it can be used as a SGS to predict the flow dynamics of the wake behind a turning ship.

\subsubsection{Properties of turbulent ship wakes}

The mean inflow boundary data was obtained by slicing the RANS solution at $\mathrm{x} / \mathrm{L}=1.5$ plane for turning ship wake and $\mathrm{x} / \mathrm{L}=1.05$ for the non turning ship wake and then interpolated to the inlet plane of the computational domain. The mean axial velocities for both non turning and turning ship wake after the interpolation are presented in Figure 3.19 (a) and (b). To see the effect of the LES wake calculation only, the axial velocity 
contours are adjusted due to rotation and the mean axial velocity adjusted for rotation at the IDP $(\mathrm{x} / \mathrm{L}=1.5)$ for turning ship wake is given in Figure 3.19 (c). By using RFG and the mean flows on these planes, the turbulent inflow boundary for both ship simulations was reconstructed. Figure 3.20 presents the pseudo random flow field generated by the RFG on the IDP for turning ship wake. For both non-turning and turning ship wake simulations, the physics of the turbulent wakes have been investigated. Results from the simulations are compared with the straight wake measurements previously studied by Hoekstra \& Ligtelijn (1991). From the axial velocity contours in Figure 3.21(a), two stable large bilge type vortices are observed for the non-turning ship wake simulation using the standard Smagorinsky model $\left(\operatorname{Re}=4.65 \times 10^{6}\right)$ at $\mathrm{x} / \mathrm{L}=0.65$. Two small side vortex pairs are observed away from the center of the wake. These vortex pairs drift in the spanwise direction, get weaker and eventually disappear. From the axial velocity contours adjusted for rotation at $x^{\prime} / L=0.65$ for the turning ship wake in Figure 3.21(b), one large bilge vortex, that moves downwards, is observed and is probably the result of merger of the two vortices (bilge vortices of opposite rotation) under the action of Coriolis and centrifugal forces for the turning ship wake simulation. There is a smaller circulation region (a side vortex) on the outer rim of the wake. The streamwise flow field causes the side vortex to weaken and the wake decays in the outer region of the near wake similar to non-turning ship. The Coriolis force seems to generate more energetic and less diffusive eddies, hence it seems to increase kinetic energy content of the wake (Yavuz et al., 2002). 

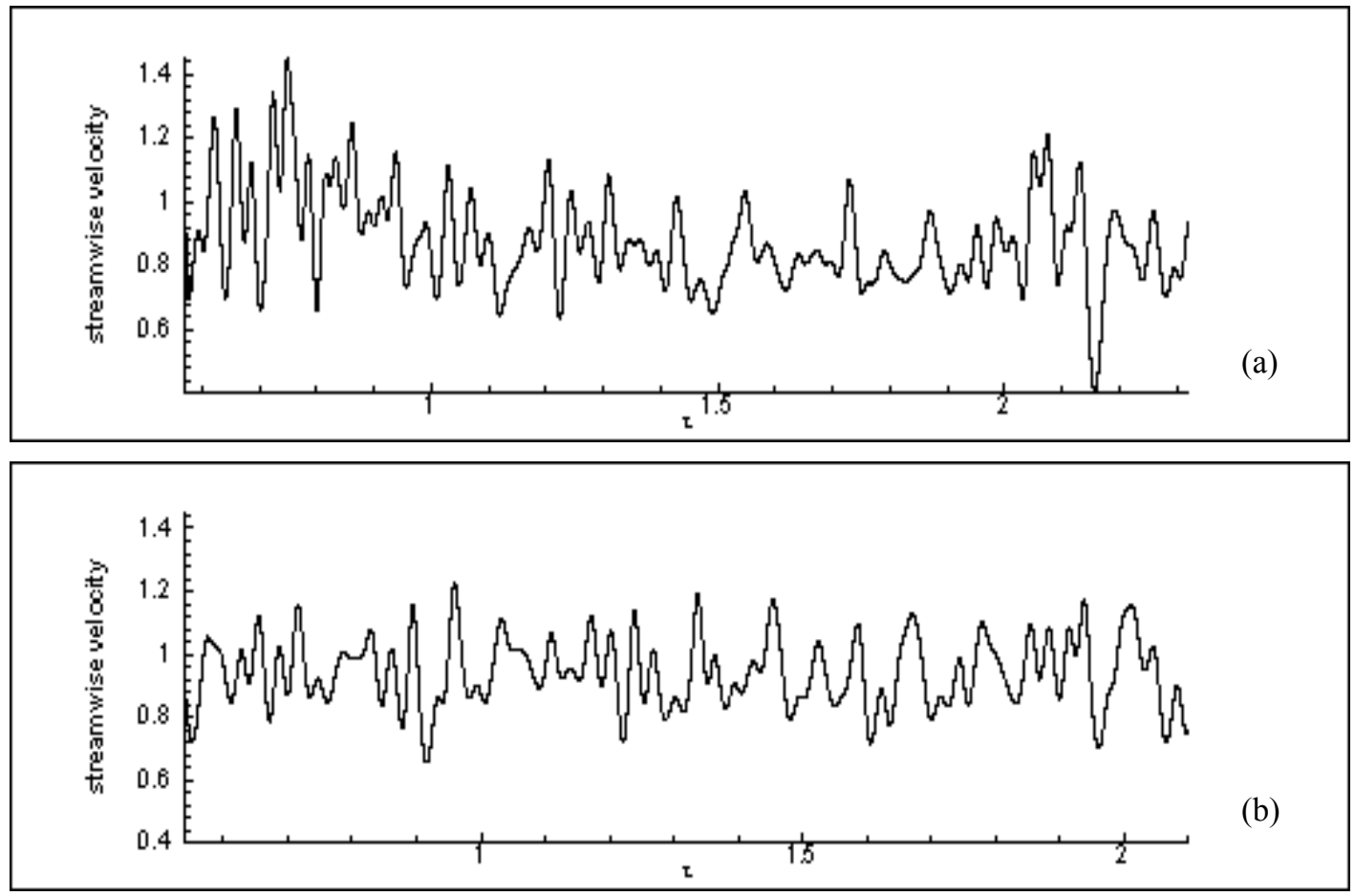

Figure 3.18. Temporal history of streamwise velocity components at $10^{\circ}(x=1.02, y=-$ 0.001 and $\mathrm{z}=-3.18$ ) (finest grid) and $\mathbf{C}_{\mathbf{s}}=\mathbf{0 . 0 5 2}$ a) Case-1 b) Case-2 
Figure 3.22 shows a comparison of the predicted velocity contours with the macro wake measurements at $\mathrm{x} / \mathrm{L}=1.20$ by Hoekstra \& Ligtelijn (1991) for the ship model No. $5452\left(\operatorname{Re} \sim 1 \times 10^{7}\right)$ with the non-turning ship wake at the same location. For both studies, minimum axial velocity occurs near the free surface of the center of the wake, as seen in Figure 3.22. Both axial velocity contours look like an upside down rimmed hat form. Two side lobes close to the free surface and a central lobe are observed. As stated by Hoekstra \& Ligtelijn (1991), these contour forms are very much alike for all ship hulls. Figure 3.22 indicates that from the straight ship wake simulation, similar physics are obtained when compared with the macro wake measurements at the same location. Moreover, the extent of axial turbulence intensities is also in reasonably good agreement with measurements (Hoekstra \& Ligtelijn, 1991), as shown in Table 3.1. Figure 3.23 shows the axial development of the wake of a turning ship. The bulk movement of this one large bilge vortex can be seen very well through the wake as it moves under the action of the Coriolis and centrifugal forces. The comparison of the energy spectra of the velocity fluctuations for the two wakes is shown in Figure 3.24. It is seen that the turning ship wake has more energetic fluctuating eddies (the values are almost 10 times higher) as compared to the non-turning ship at the same location. This finding is expected as the initial value of the kinetic energy for the non-turning ship wake is almost 8 times smaller than the turning ship wake. For the turning ship wake, the smallest eddy turnover has been calculated to see whether the numerical time step is correlated or not with the time step used in the simulation; $\Delta \mathrm{t}=1 \mathrm{e}-04$. Hence, the smallest eddy turnover (numerical or discretization turbulence) can be defined as, smallest eddy size over the ship velocity, where smallest eddy size is $2 \sqrt{\Delta x_{1} \Delta x_{2} \Delta x_{3}}$ and the ship velocity is 1 . At $10^{\circ}$, 

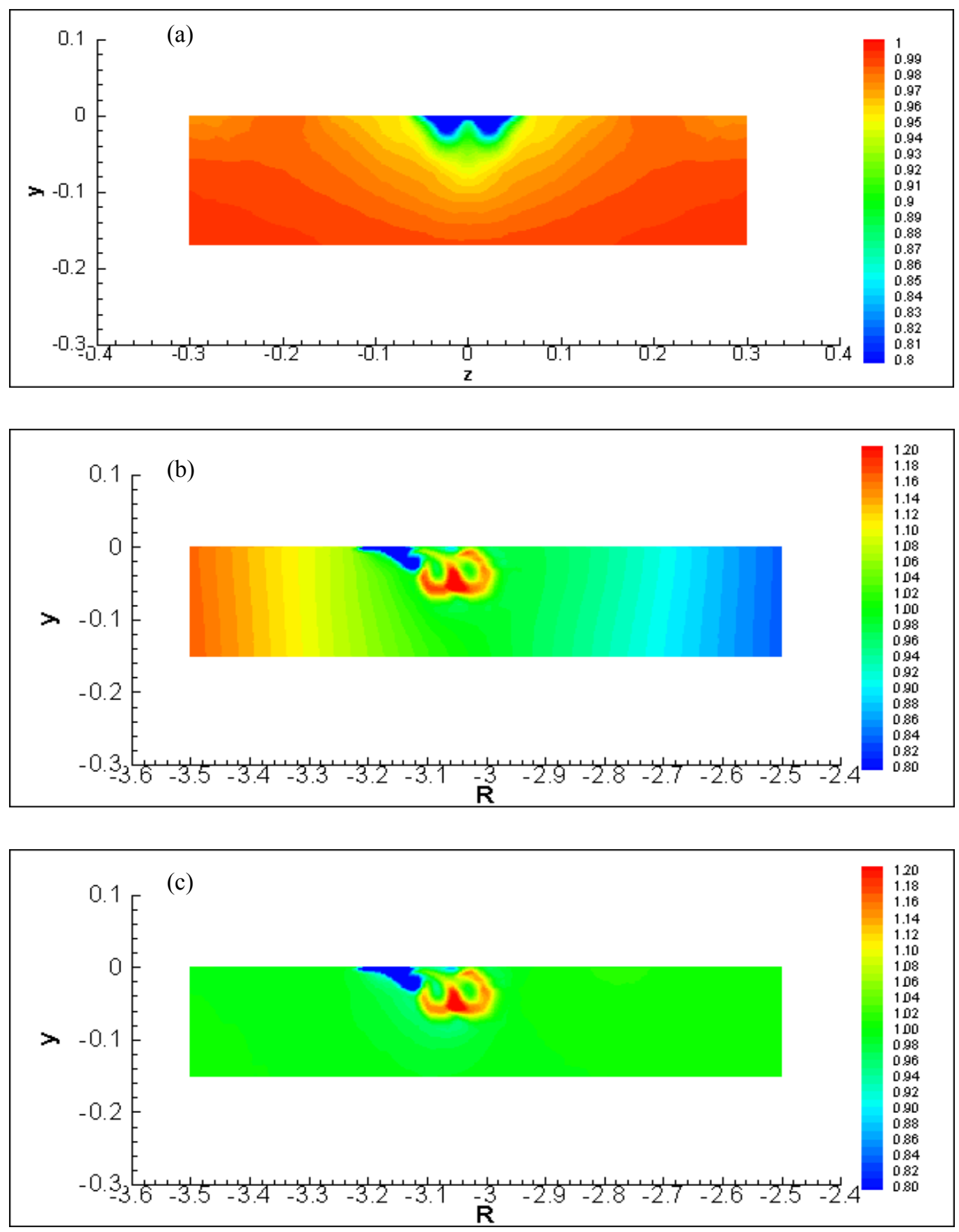

Figure 3.19 a) The mean axial velocity contour at the inlet data plane (IDP) for nonturning ship (Shi et al. 2001) (finest grid) b) The mean axial velocity contours at the IDP for turning ship wake (finest grid) c) The mean axial velocity contours after subtracting solid body rotation contribution at the IDP for turning ship wake (finest grid) 

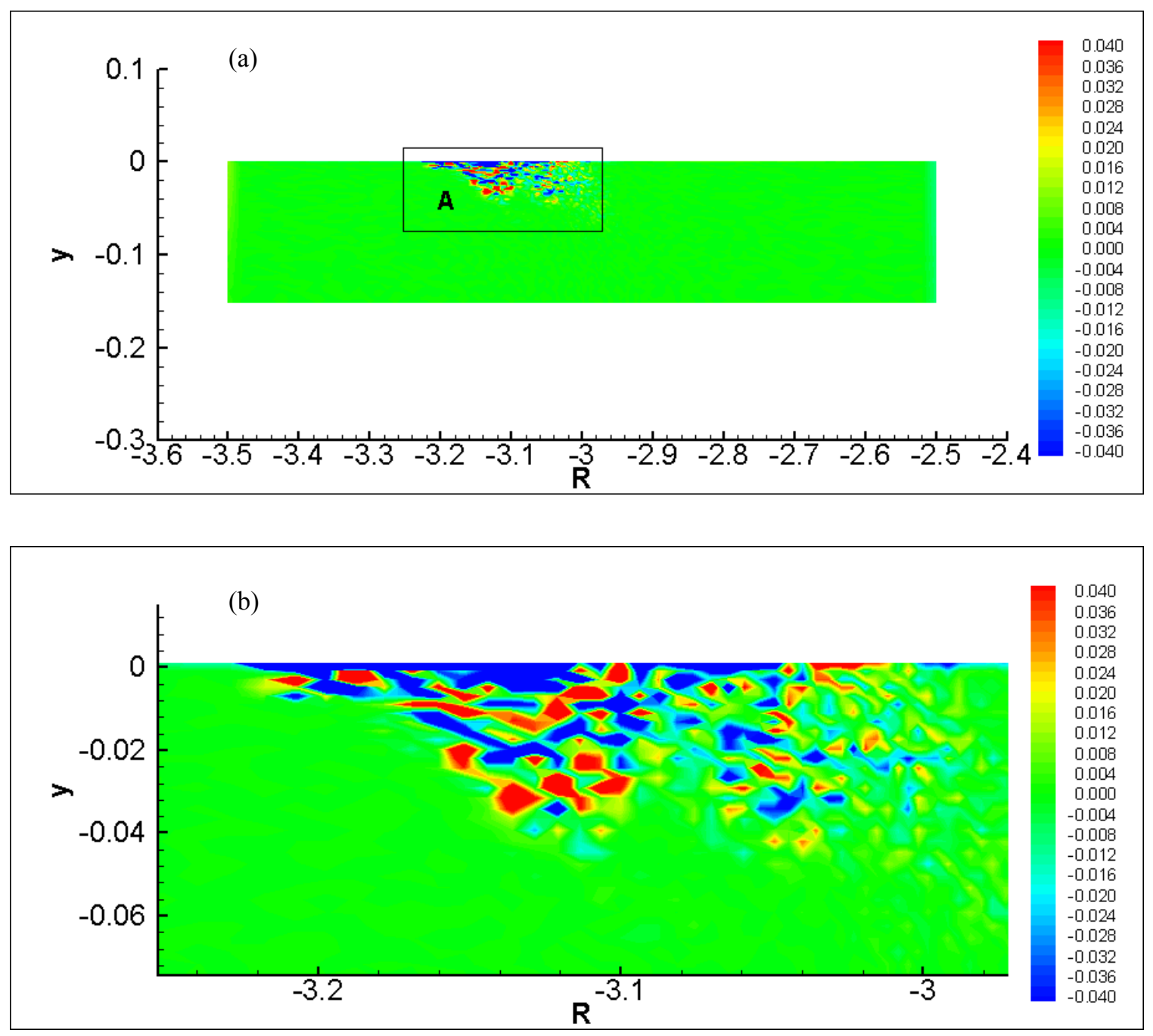

Figure 3.20. a) The axial flow field provided by RFG at the inlet plane (IDP) for turning ship wake b) Enlarged view of A (finest grid) 

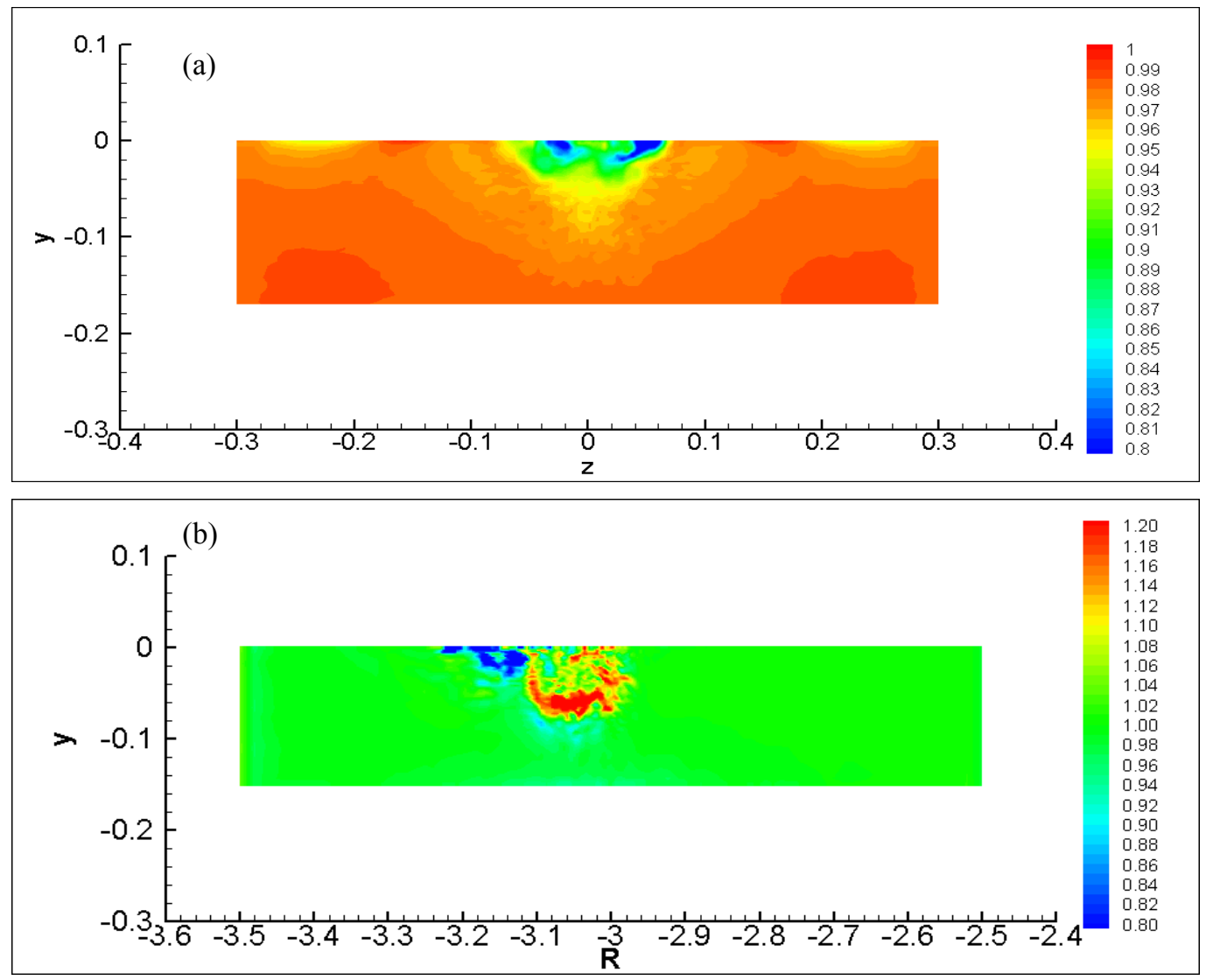

Figure 3.21. a) Axial velocity contour at $\mathrm{x} / \mathrm{L}=0.65$ for non-turning ship (Shi et al. 2001) (finest grid) b) Axial velocity contours after subtracting solid body rotation contribution at $x^{\prime} / L=0.65$ for turning ship wake (finest grid) 

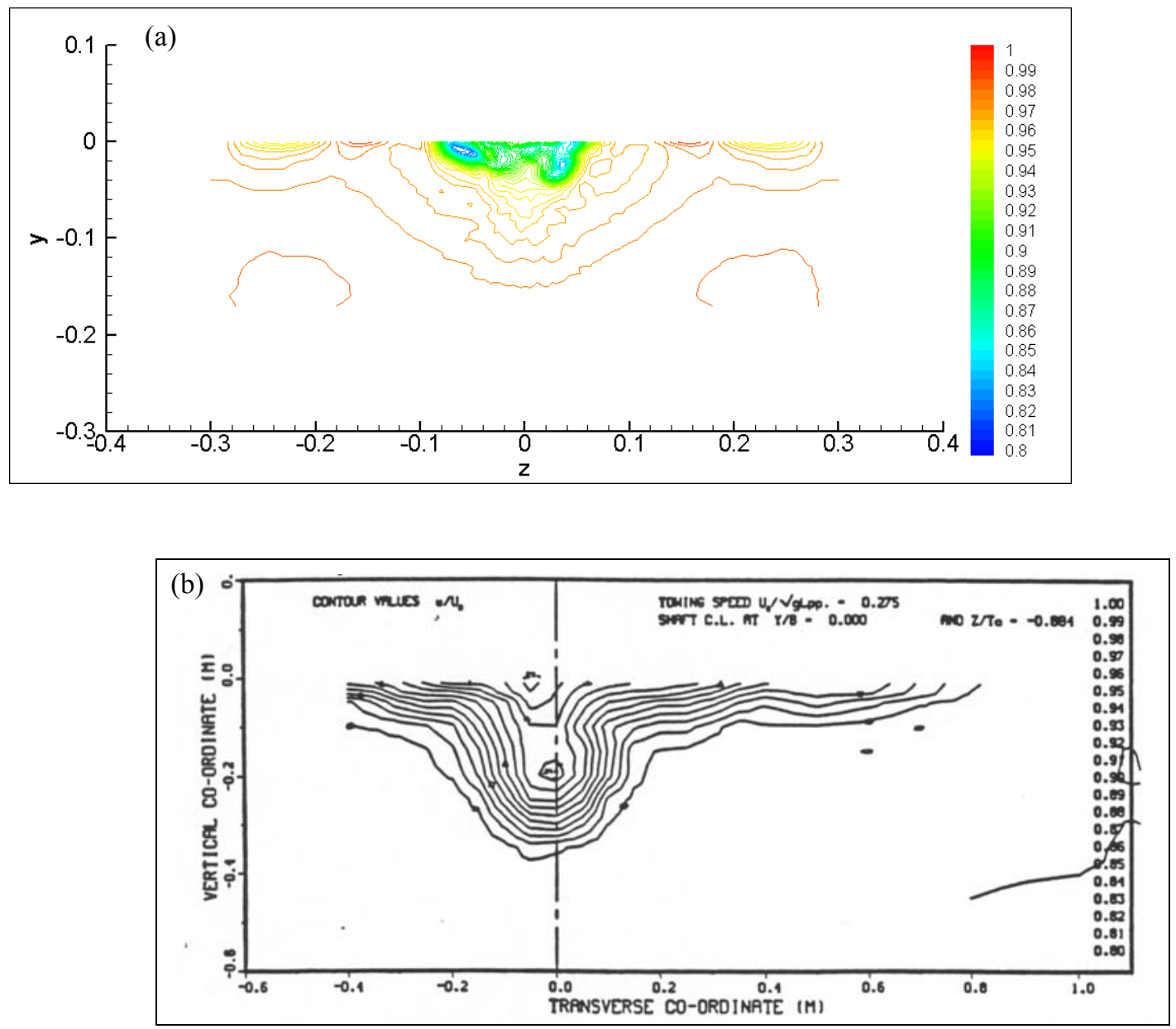

Figure 3.22. a) Predicted velocity contours at $\mathrm{x} / \mathrm{L}=1.20$ for non-turning ship-(Shi et al. 2001) (finest grid) b) Macro wake measurements at $\mathrm{x} / \mathrm{L}=1.20$ by Hoekstra \& Ligtelijn (1991) for ship model No. $5452\left(\operatorname{Re} \sim 1 \times 10^{7}\right)$ 


\begin{tabular}{|c|c|c|}
\hline \multicolumn{3}{|c|}{ for any ship hull - maximum value of root mean square fluctuations } \\
\hline location & experimental & numerical \\
\hline & $(\mathrm{m} / \mathrm{s})$ & (values obtained $\left.{ }^{*} \mathrm{U}_{\mathrm{s}}\right)(\mathrm{m} / \mathrm{s})$ \\
\hline $\mathrm{x} / \mathrm{L}=0.25$ & $0.106 \pm 0.02$ & 0.083 \\
$\mathrm{x} / \mathrm{L}=0.6$ & $0.067 \pm 0.01$ & 0.069 \\
$\mathrm{x} / \mathrm{L}=1.0$ & $0.047 \pm 0.01$ & 0.057 \\
\hline
\end{tabular}

Table 3.1. Comparison of the maximum values of the root mean square fluctuations for some locations in units of $(\mathrm{m} / \mathrm{s})$ for non-turning ship 
$\Delta \mathrm{x}_{1}=4.523 \times 10^{-3}, \Delta \mathrm{x}_{2}=2.779 \times 10^{-3}, \Delta \mathrm{x}_{3}=4.07 \times 10^{-3}$, then the smallest eddy turnover is calculated to be $4.52 \times 10^{-4}$, where the frequency, $\mathrm{f}$, is 1 over $4.52 \times 10^{-4}$, which equals to $2520 \mathrm{~Hz}$. Since the numerical time step is calculate to be much larger than the approximately found $30 \mathrm{~Hz}$, then it is found to be not correlated with the time step, $\Delta \mathrm{t}$. It should also be noted that the shape of spectra are obtained to be quite different for turning ship case. In addition to these, the wake spreading or wake width for the non-turning ship, which is obtained to be roughly $\mathrm{w} \sim \mathrm{x}^{1 / 4}$ is consistent with Buller \& Tunaley (1989)'s

measurements. Milgram et al. (1993) and Hoekstra \& Ligtelijn (1991) found $w \sim x^{1 / 5}$. The spreading rate of the turning ship wake can't be easily observed like in the non-turning ship. This is also seen from the predicted vertical vorticity contours in Figure 3.25. It may be due to the grid coarsening towards the end of the calculation domain. More, the "inboard" side of wake has a much sharper edge while the "outboard" side is more diffusive. This might be due to instability in the "outboard" side.

\subsection{Assessment of SGS models}

As explained before, the anisotropy of the turbulence observed near a free surface can not be obtained by a standard Smagorinsky model (SMG). Therefore, in Section 2.3, the SMG model is improved with free surface modifications and also a nonlinear oneequation model is formulated. The purpose of this next study is therefore to compare and discuss the performance of existing and improved SGS models. For this reason, the fully developed open channel flow case is used as verification for the effects of the free surface modifications before applying these models to the surface ship wake. The geometry of 

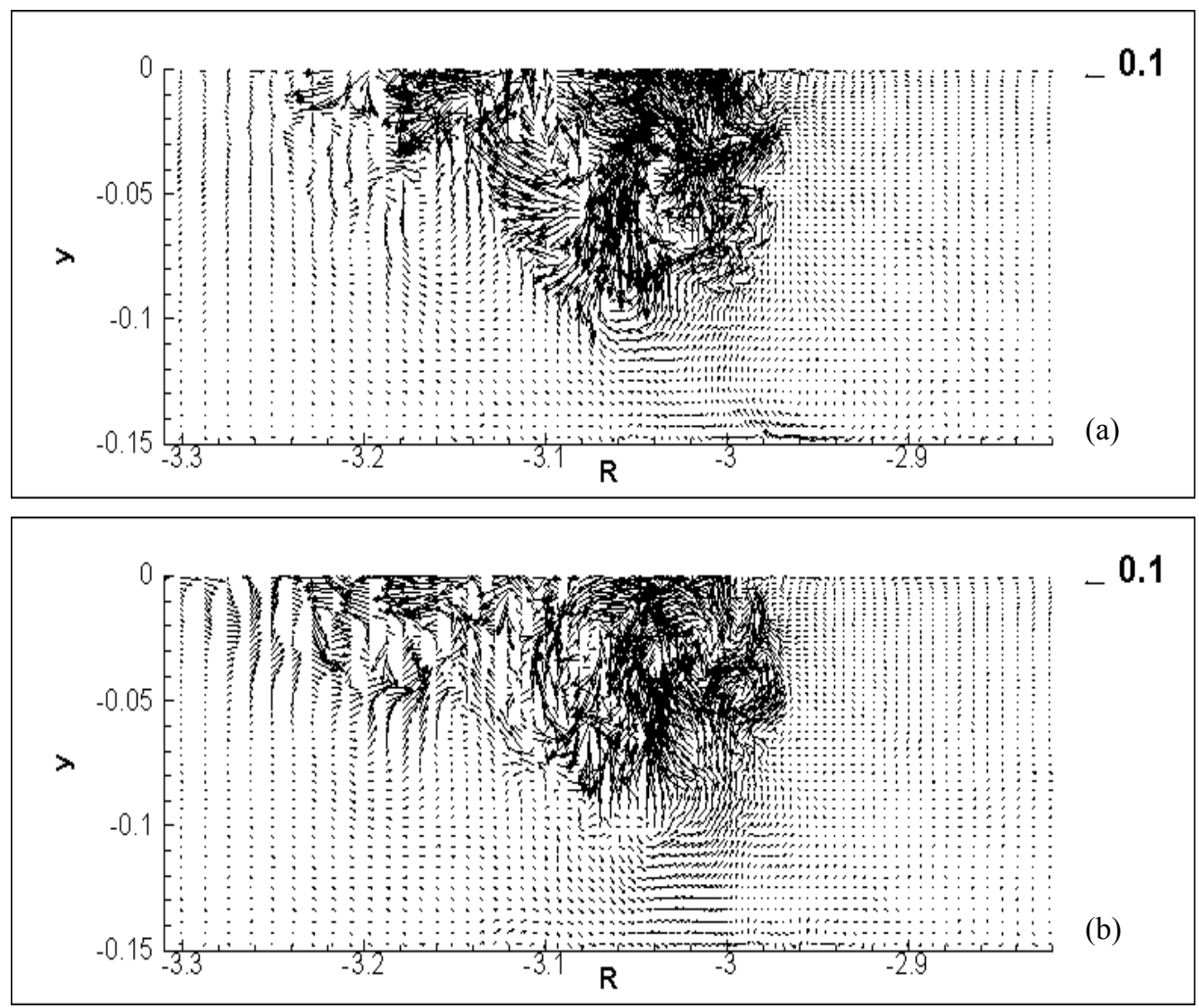

Figure 3.23. Velocity vectors for turning ship using standard Smagorinsky model (finest grid) on a vertical plane at an angle of a) $5^{\circ}\left(\mathrm{x}^{\prime} / \mathrm{L}=0.8\right)$ b) $10^{\circ}\left(\mathrm{x}^{\prime} / \mathrm{L}=1.02\right)$ 

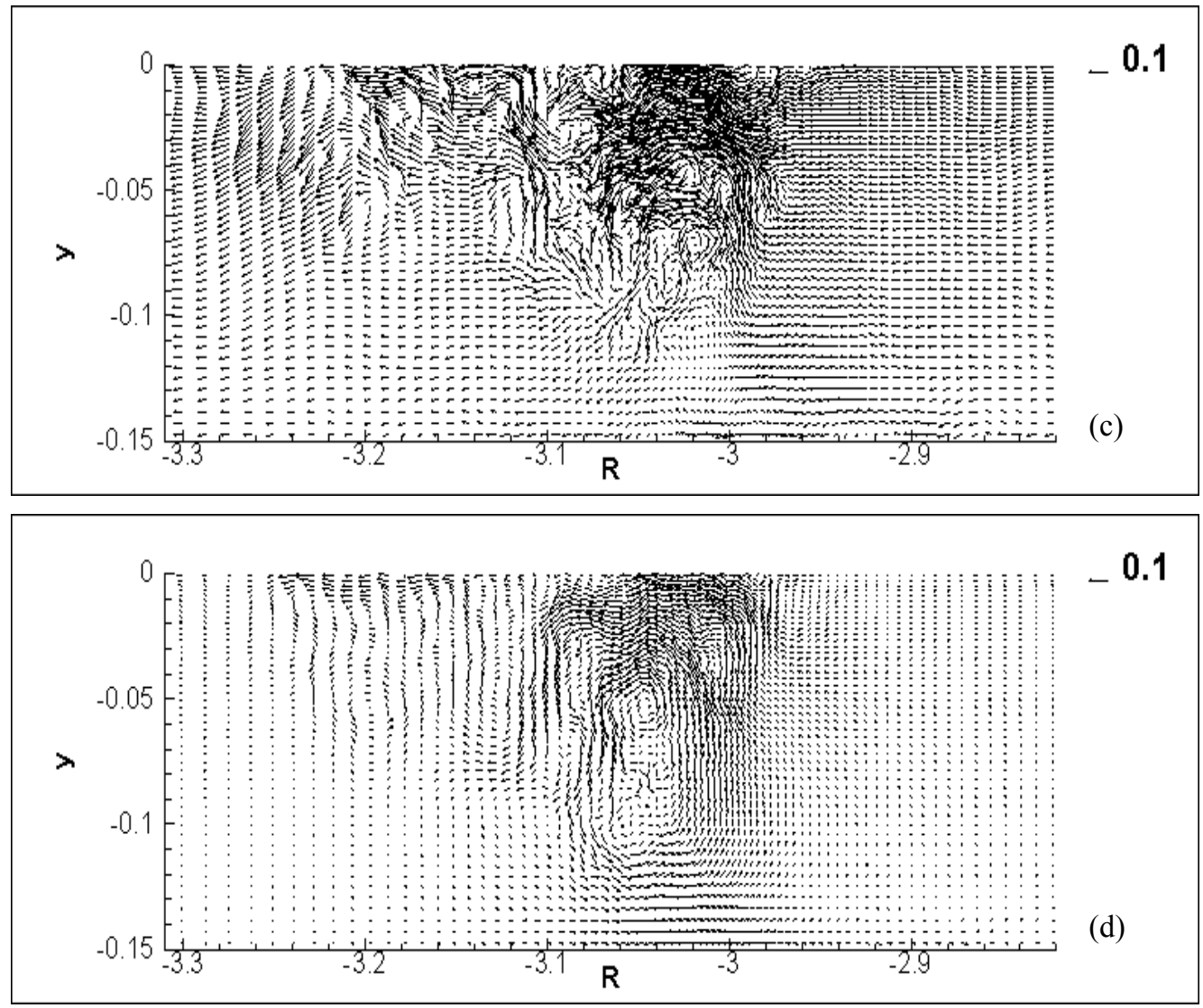

Figure 3.23. Velocity vectors for turning ship using standard Smagorinsky model (finest grid) on a vertical plane at an angle of c) $20^{\circ}\left(x^{\prime} / L=1.55\right)$ d) $30^{\circ}\left(x^{\prime} / L=1.75\right)$ 

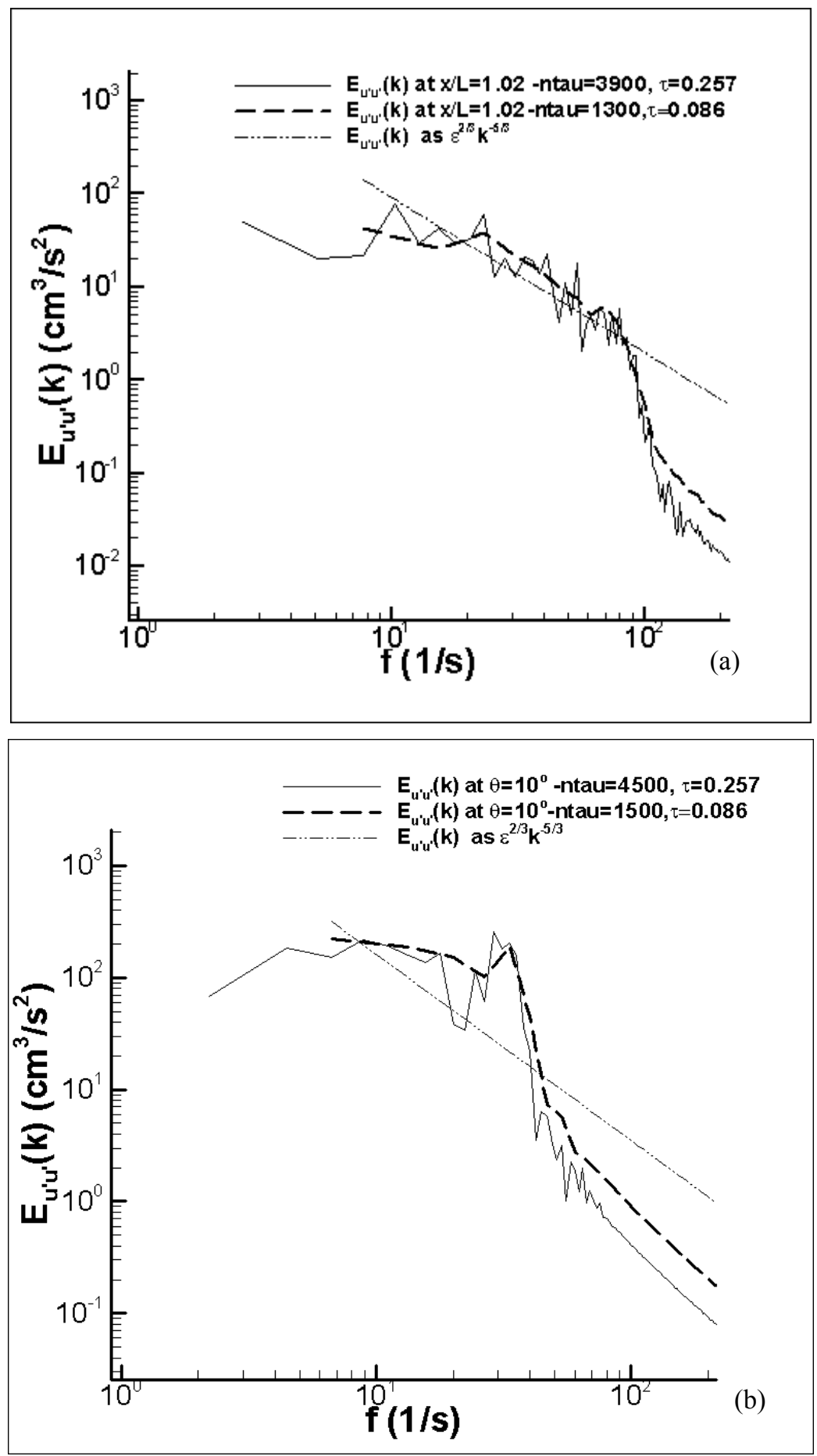

Figure 3.24. Energy spectra of the velocity fluctuations a) Non-turning ship (Shi et al. 2001) (finest grid) b) Turning ship (finest grid) 

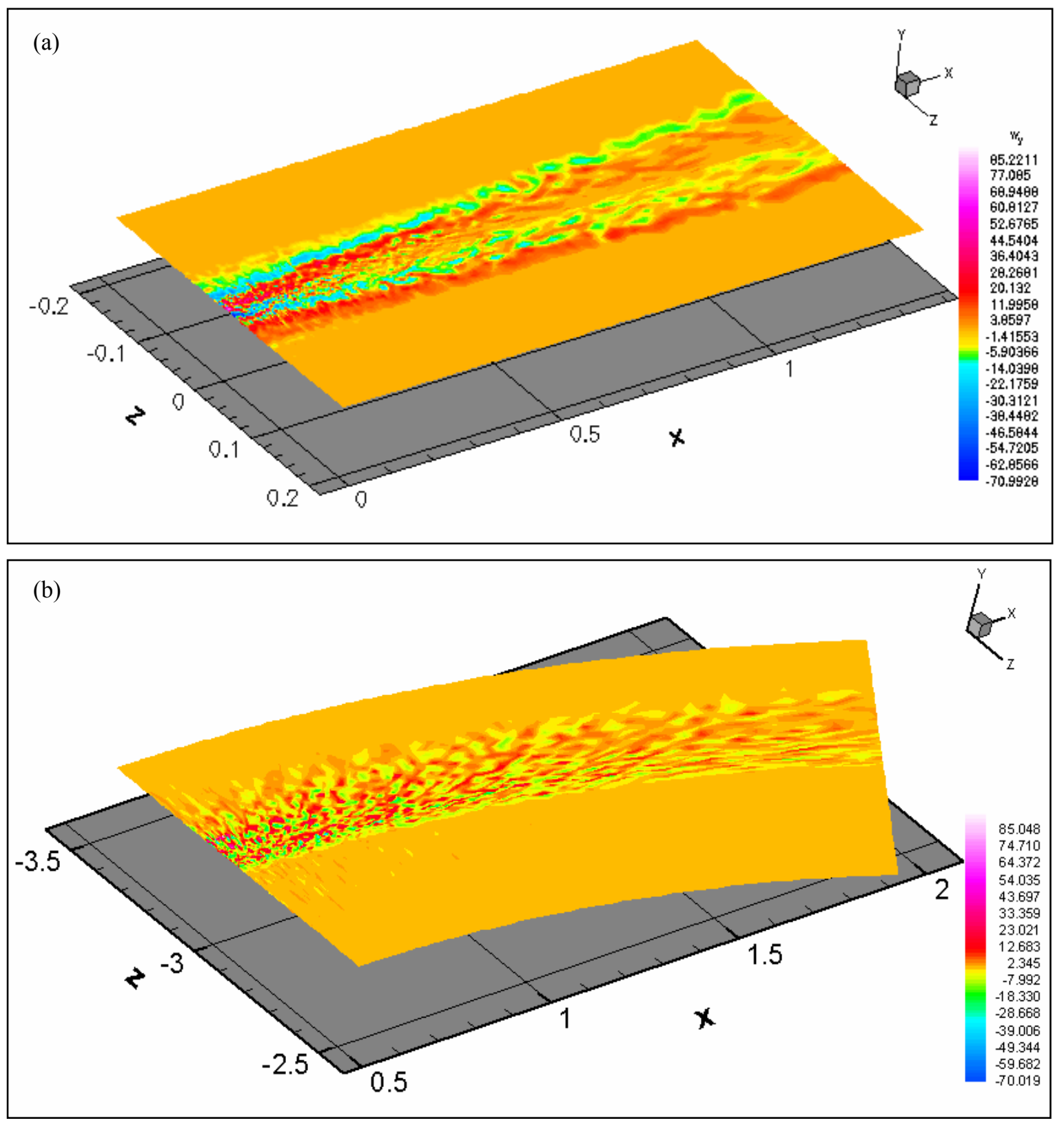

Figure 3.25. Predicted vertical vorticity $\left(\omega_{\mathrm{y}}\right)$ for a) Non-turning ship (Shi et al. 2001)

(finest grid) b) Turning ship wake (finest grid) (Case 2) 
the channel flow is shown in Figure 3.26. The Reynolds number is 67000 and Froude number is 0.5 . A grid of 66x66x66 with nonuniform distribution in the vertical direction has been used. The grid distribution is selected in the y-direction in such a way to resolve the viscous sublayer $(y+<5)$ (Moin and Kim, 1982). The no-slip boundary condition is used for the bottom wall, whereas the top surface has been represented by the symmetry boundary condition. In the spanwise and horizontal directions, periodic boundary conditions are applied. The Smagorinsky model with $\mathrm{C}_{\mathrm{s}}=0.2$ together with a central differencing scheme for the calculations is used as it has been shown to give good results (Shi, 2001). The turbulence intensities are normalized by the friction velocity, $u_{\tau}$, $\left(\mathrm{u}_{\mathrm{rms}}=\sqrt{\overline{\overline{u^{\prime 2}}}} / u_{\tau}\right)$ and $u_{\tau}$ is calculated from $\tau_{w}=\sqrt{\frac{u_{\tau}}{\rho}}$ where $\tau_{w}=\left.\mu \frac{\partial \bar{u}}{\partial y}\right|_{w}$.

The turbulence intensities calculated with the Free Surface Modification (FSM) are compared with other experimental and numerical studies. The experimental study by Komori et al. (1990) measured the turbulence quantities using a two-color laser Doppler velocimeter (LDV). Lam and Banerjee (1992) applied DNS to an open channel flow and compared their results to the experiments. The free surface was treated as a rigid slip surface and their DNS was based on a Fourier-Chebyshev pseudo spectral method using $32 * 64 * 65$ grid points. Komori et al. (1993) also applied DNS based on a fifth order finite difference formulation using $60 \times 60 \times 40$ for a dimensionally $10 \delta \times \delta \times 5 \delta$ open channel flow, where $\delta$, here, represents the open channel height. A non-uniform mesh was used, which was refined in the wall and the free surface regions. Komori et al. (1993) compared the predicted results with Komori et al.'s (1990) experimental and Lam and Banerjee's (1992) numerical results. Shi et al. (2000) studied the free surface effects in open channel 


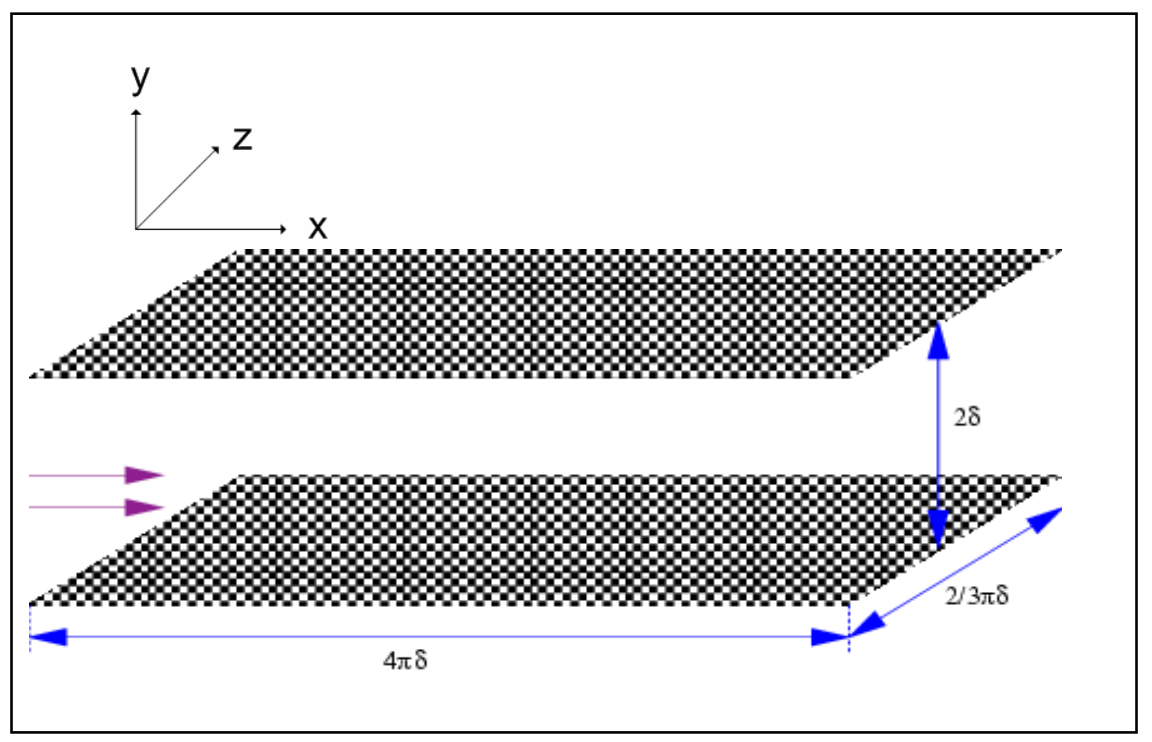

Figure 3.26. Geometry of the open channel flow case

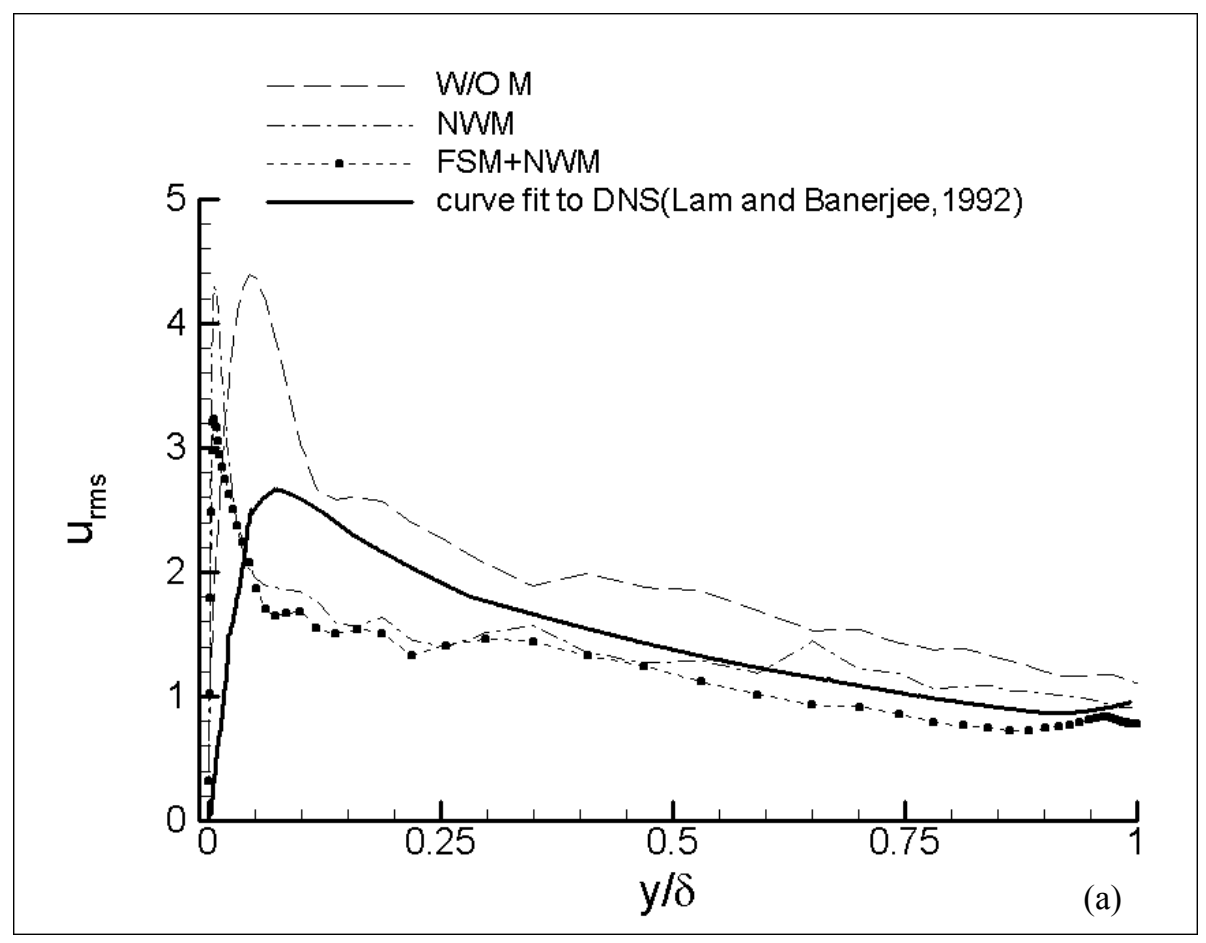

Figure 3.27. Resolved turbulence intensities in a) streamwise direction: (values are normalized with $\mathrm{u}_{\tau}$ ) 

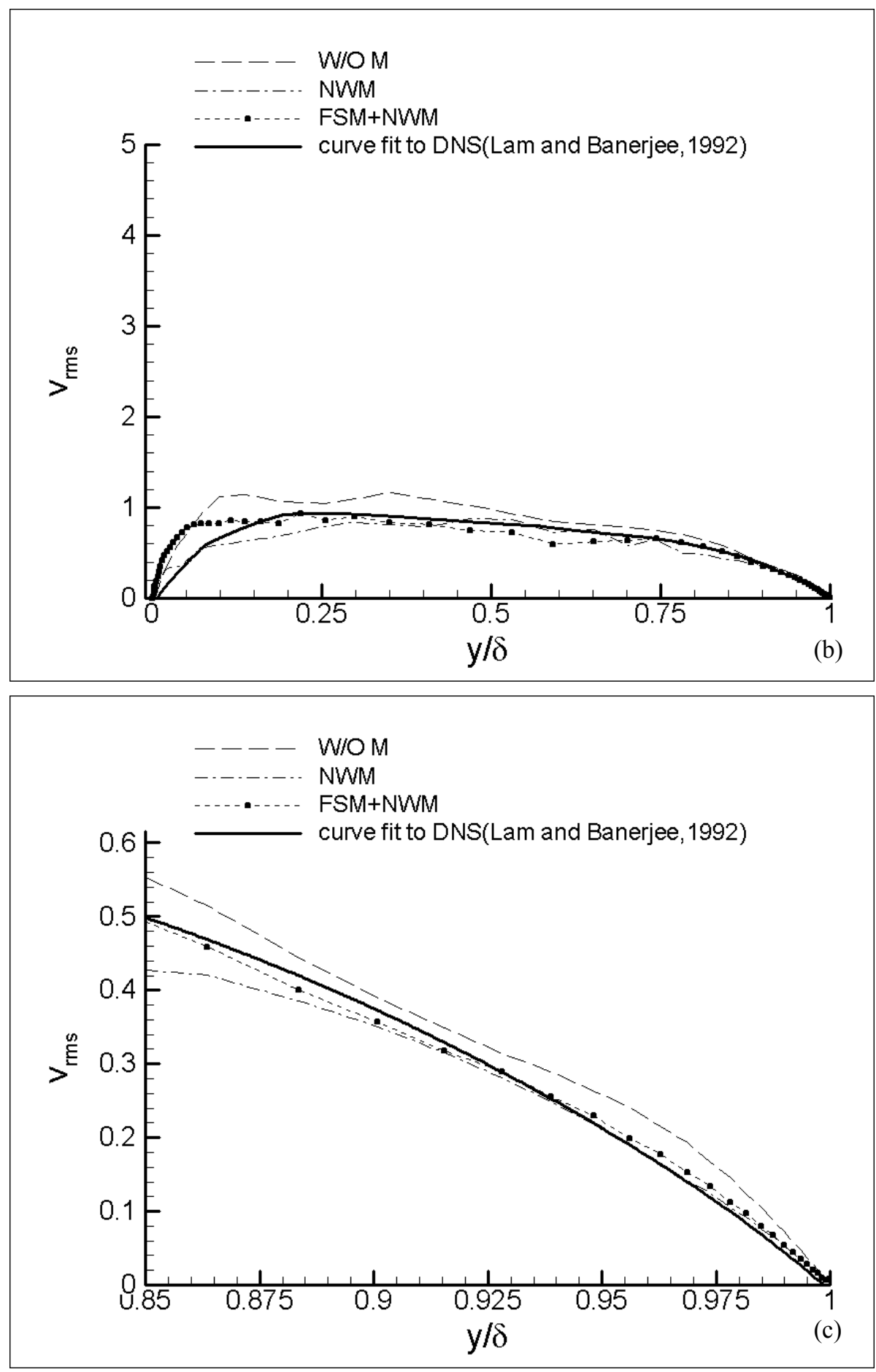

Figure 3.27. Resolved turbulence intensities in b) vertical direction; c) vertical direction enlarged near the free surface region :(values are normalized with $\mathrm{u}_{\tau}$ ) 


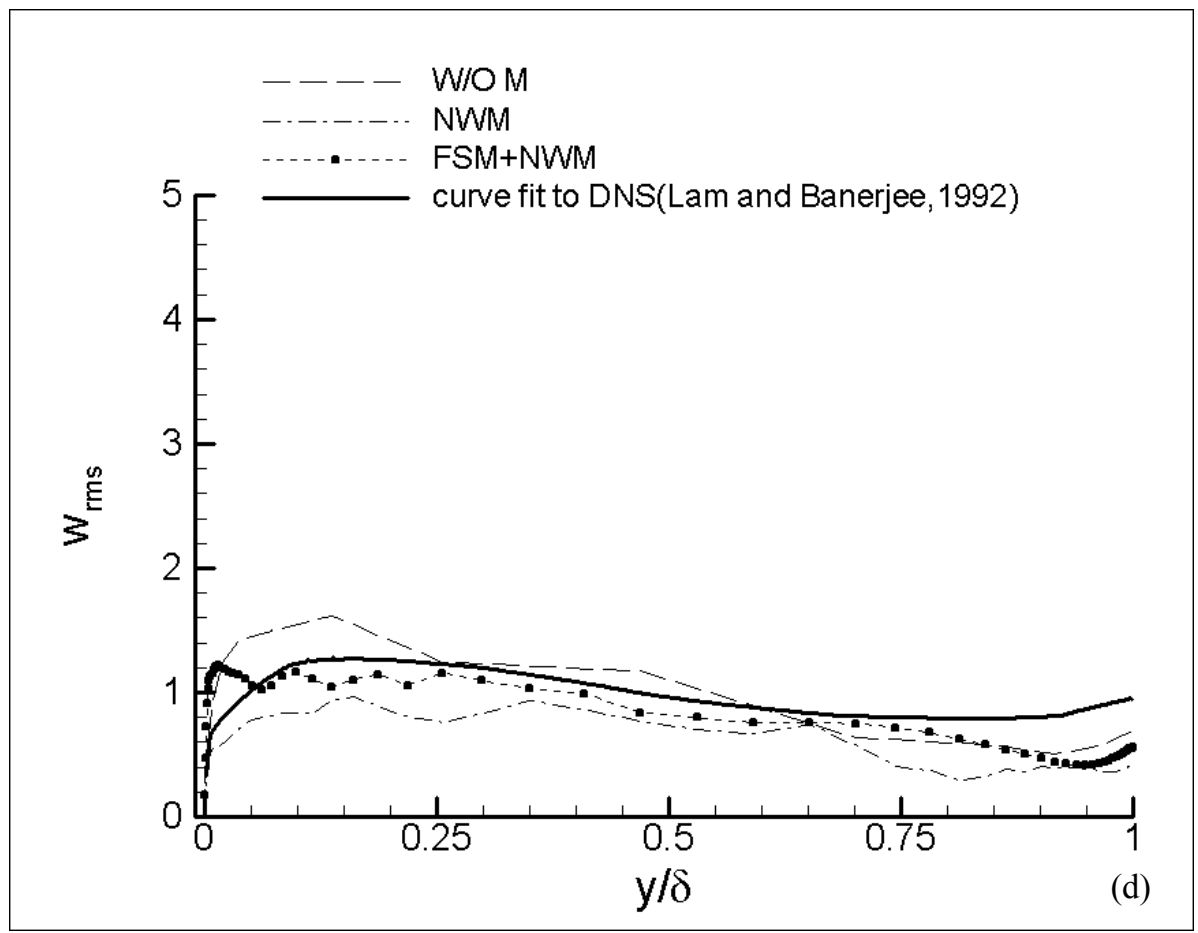

Figure 3.27. Resolved turbulence intensities in d) spanwise direction: (values are normalized with $\mathrm{u}_{\tau}$ )

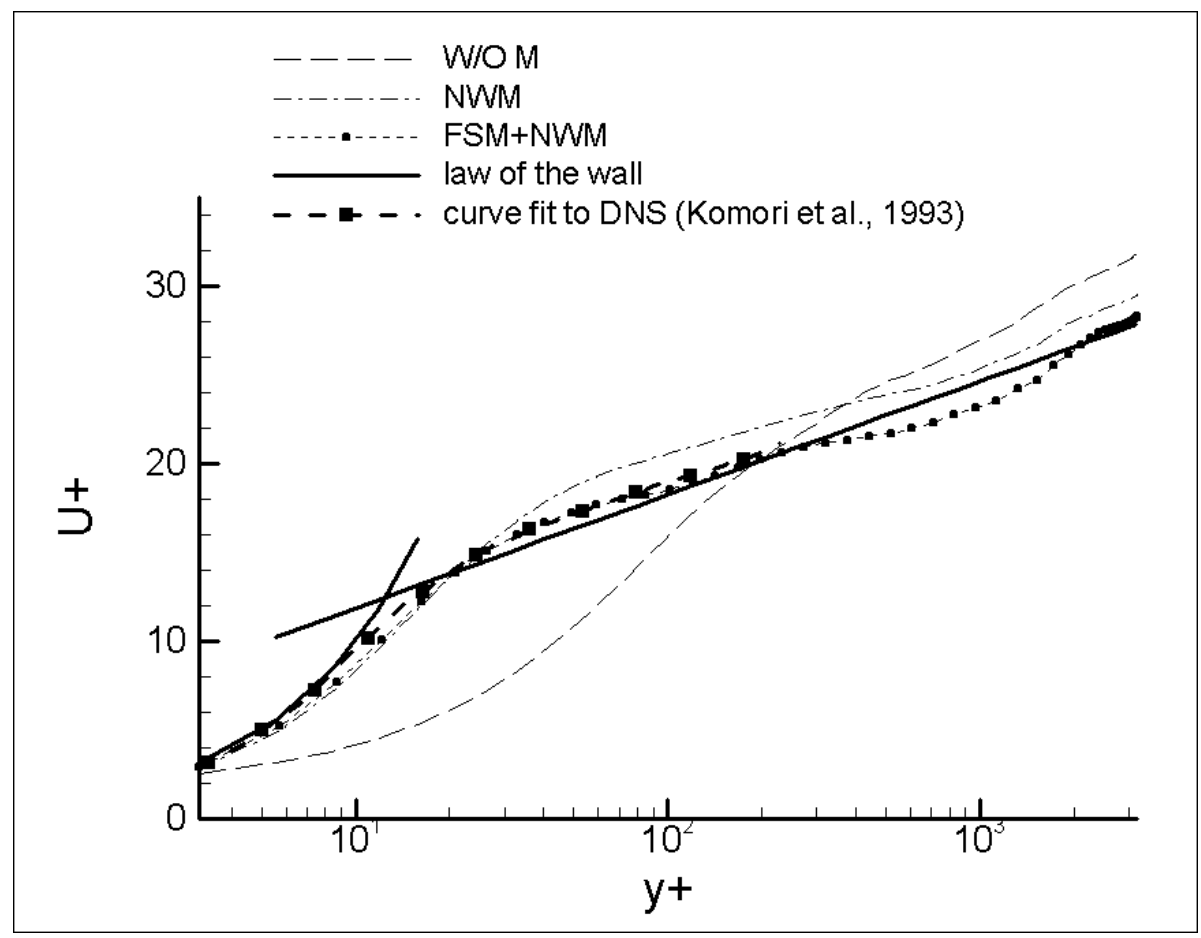

Figure 3.28. Comparison of the mean velocity profile 
flow with a deformable free surface for $\mathrm{Fr}=0.66$ and $\mathrm{Re}=20800$ with a computational domain of $6 \delta \times 4 \delta \times \delta$ size using $64 \times 64 \times 58$ grid points. They also used Van Driest damping effect near the wall.

Figure 3.27 depicts the predicted resolved turbulence intensities for three different models; W/O M (without modification), only NWM (near wall modification) and FSM+NWM (free surface and near wall modification). Figure 3.27 (a) shows that the predicted streamwise turbulence intensity is reduced using NWM and the maximum values are shifted towards the wall when compared to the results of $\mathrm{W} / \mathrm{O} \mathrm{M}$. When FSM+NWM is compared to the cases with NWM and W/O M, it is seen that in the near wall region, the predicted turbulence intensity peak is lower and the value is closer to the DNS results of Lam and Banerjee (1992). The case without modifications (W/O M) predicts the streamwise intensities much higher than the DNS results. When NWM is applied, the increase slightly diminishes and the values get closer to the DNS results. However the predicted streamwise intensities from FSM+NWM is much closer to the DNS predictions than those obtained from W/O M and NWM, as observed in Figure 3.27 (a). Near the wall region, the peak value differs in all models, however, FSM+NWM predicts the closest values to the DNS results when compared to the other two cases. In all three cases, the predicted vertical fluctuations (see Figure 3.27 (b)) near the free surface decrease approximately to zero. However, in the free surface region $(y / \delta>0.85)$, FSM+NWM predicts turbulence intensities, which are much closer to the predictions of Lam and Banerjee (1992) than the other two models, as observed in Figure 3.27 (c). For all three models, the spanwise and the streamwise intensities increase slightly whereas the vertical intensities decrease near the free surface, as previously observed by 
Nakayama and Yokojima (2002) and Komori et al. (1993). This indicates that the extra energy is redistributed to the horizontal and spanwise components of the velocity via pressure fluctuations.

Overall, it can be concluded that with using FSM+NWM, the levels of turbulence intensities are in better agreement with the DNS results (Lam and Banerjee, 1992; Yokojima and Nakayama, 2001; Nakayama and Yokojima, 2002) than with just NWM or W/O M. Then the question arises, which model is more appropriate to use for the ship wake calculations? The above discussion implies the use of FSM+NWM, especially when the predicted mean velocity profiles in Figure 3.28 are considered. The mean velocity profile is one of the important aspects to investigate the turbulent structures in unsteady flow. The predicted values of the time averaged velocity normalized by the friction velocity $\mathrm{u}_{\tau}$, for all three cases are presented against the dimensionless wall unit $\mathrm{y}+$ (Figure 3.24). Also the measurements by LDV (Komori et al., 1990) are shown which are correlated by

$$
\boldsymbol{U}^{+}= \begin{cases}\boldsymbol{y}^{+} & \left(\mathrm{y}^{+}<10\right) \\ \frac{1}{0.36} \ln \left(\boldsymbol{y}^{+}\right)+5.5 & \left(\mathrm{y}^{+}>20\right)\end{cases}
$$

In Figure 3.28, it is clearly seen that near wall modifications should be applied if the wall boundaries are present in the calculations. It's very difficult to resolve the sublayer in the wall region, especially for high Reynolds number flows (Wilcox, 1993). The length scale should guarantee the proper flow behaviour in the wall region. 
Therefore, even if the case $\mathrm{W} / \mathrm{O} \mathrm{M}$ gives relatively reasonable predictions for the spanwise and vertical turbulence intensities (note that the intensities in the flow direction are much higher than the DNS results), it should not be considered as a SGS model for wall bounded flows. Close to the free surface region, predicted values of the case with FSM+NWM are very close to the measurements (Komori et al., 1990) and the values calculated using DNS (Komori et al., 1993). In this region, the case with NWM without the FSM predicts larger velocities than the FSM+NWM predictions and the velocities

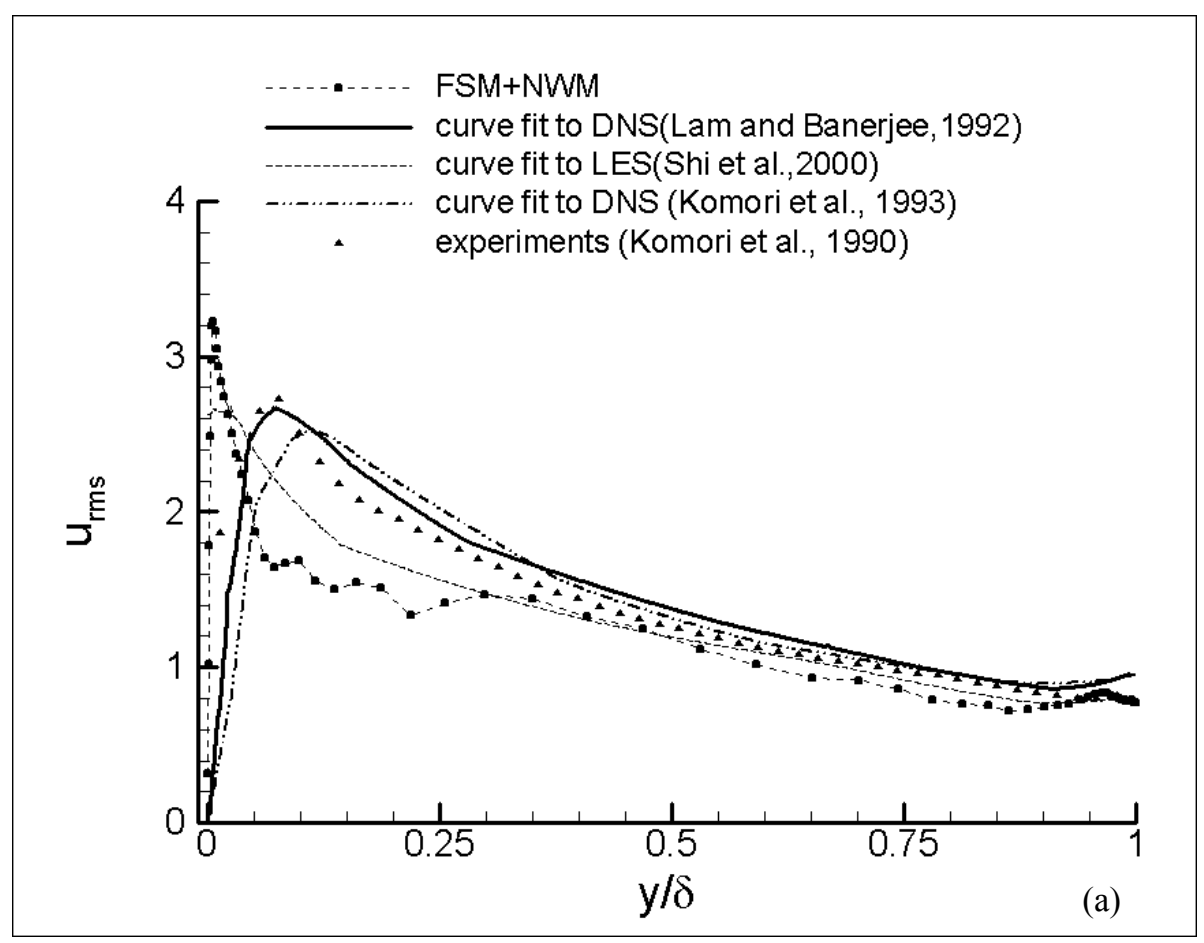

Figure 3.29 a) Vertical distributions of turbulence intensities; Comparisons between the present FSM+NWM and measurements, predictions by DNS and LES studies in streamwise direction: (values are normalized with $\mathrm{u}_{\tau}$ ) 

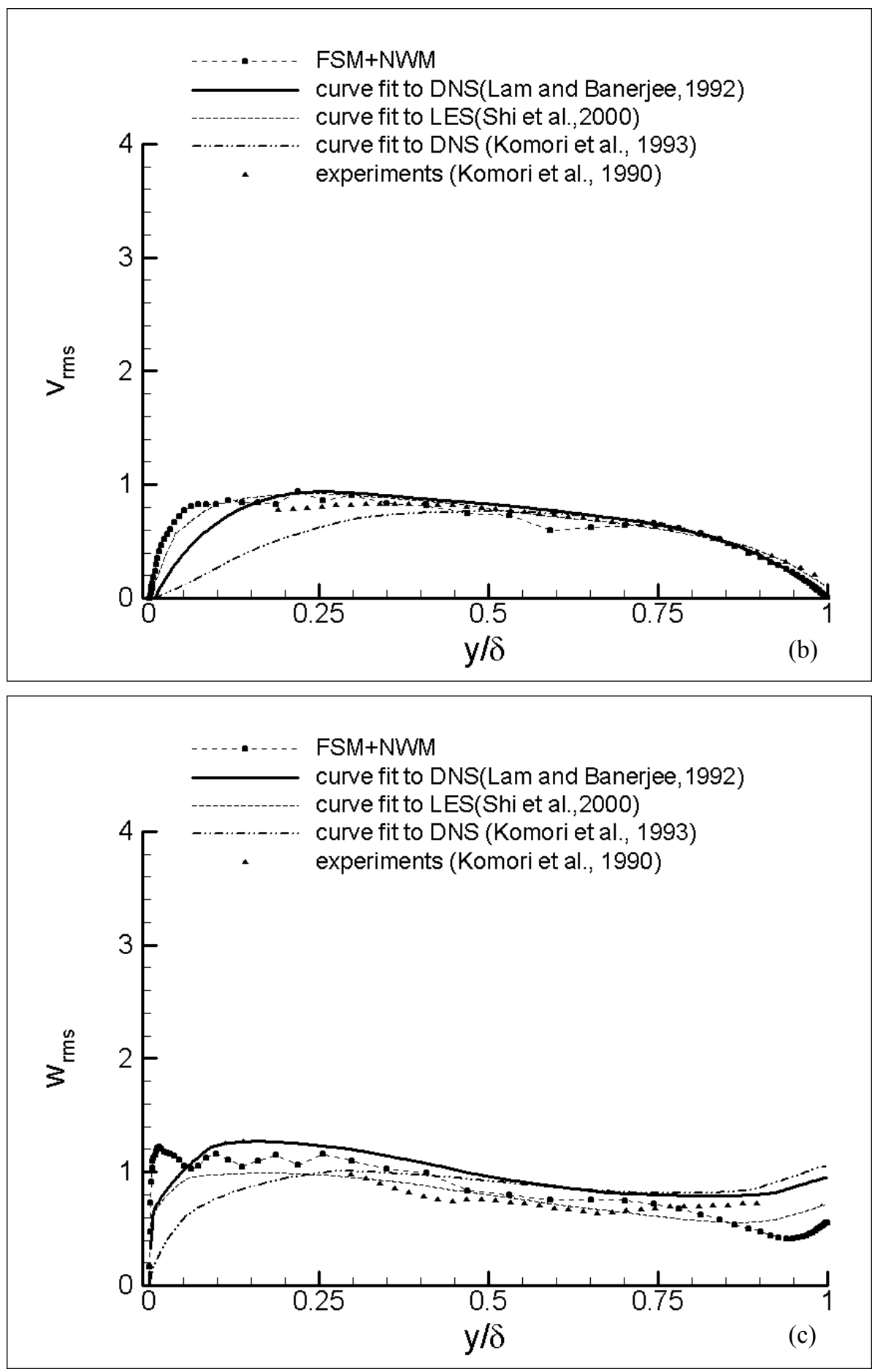

Figure $3.29 \mathrm{~b} \& \mathrm{c}$ ) Vertical distributions of turbulence intensities; Comparisons between the present FSM+NWM and measurements, predictions by DNS and LES studies in vertical and spanwise directions respectively: (values are normalized with $\mathrm{u}_{\tau}$ ) 
from measurements and DNS predictions (Komori et al. 1993), especially in the intermediate region and all through the free surface region.

Figure 3.29 compares the vertical distributions of turbulence intensities from various sources including the present simulations. It is clearly seen that there are differences between the predictions and measurements reported in previous studies. The predictions of Lam and Banerjee (1992) compare well with the measured intensities by Komori et al. (1990) rather than the DNS results of Komori et al. (1993). The differences may arise because of insufficient grid resolution (Komori et al., 1993). It is further observed that even if they increase grid resolution by c.a. $25 \%$, there are still obvious differences between measurements (Komori et al., 1990) and DNS predictions (Komori et al., 1993).

On the other hand, Shi et al.'s (2000) LES study is a good case to compare to the present simulations with FSM+NWM. As the grid width, $\Delta$, is approximately $0.046 \delta$ for Shi et al. (2000) and $0.057 \delta$ for the present study, a difference in the turbulence intensity is expected. This is observed as $y / \delta<0.25$ in Figure 3.29 (a). Then both simulations predict similar results $(y / \delta>0.25)$. Especially near the free surface $(y / \delta>0.9)$, the results from the present study are in very good agreement with Shi et al.'s (2000) predictions. Similar conclusions can be drawn for the vertical and spanwise turbulence intensity comparisons (Figure 3.29 (b) \& (c)). Throughout the vertical distribution of the channel and especially very near the free surface region, the predicted vertical intensities are in good agreement with the results from all studies except the DNS study of Komori et al. (1993). However, it is seen that the results from that DNS study differs from the other measurements and predictions as $\mathrm{y} / \delta<0.5$. Moreover, the predicted spanwise turbulence 
intensities have the same trend as the LES predictions of Shi et al. (2000). However, very close to the free surface region, $(y / \delta>0.9)$, the values are slightly less than those obtained by LES (Shi et al., 2000).

The main goal of the present study concerning SGS models is to find one model that can be used to simulate the physics of the air bubbles in the wake of the ship. Near the free surface, turbulence plays an important role especially in the dispersion of these bubbles. Good SGS models are then important. Even near the free surface, certain physical mechanisms occur. It is believed that this can be best accomplished by employing a one equation model (OEM). The independent definition of the velocity scale results in a more accurate prescription of the SGS time scale compared to algebraic eddy viscosity models (Piomelli, 1999). Very near the surface, the turbulence length scale approaches zero (damped by free surface). This region is usually not well resolved. The OEM directly takes the transport of subgrid stresses into account for these regions. The application of OEM to channel flows has been studied before and reasonably good results have been presented (Shi, 2001). Comparing to linear OEM, non-linear one equation models (NOEM) are expected to better represent the anisotropy occurring near the free surface. Knowing that the OEM was verified earlier, a study using NOEM+NWM has been started for an open channel flow. Figure 3.30 shows a comparison of the turbulence statistics normalized by the wall shear velocity, $u_{\tau}$ of the two simulations using NOEM+NWM and FSM+NWM. The results indicate that NOEM+NWM match the LES (Shi et al., 2001) results fairly well starting from the middle section of the channel and throughout the free surface region. In addition, a closer agreement to the DNS study of 

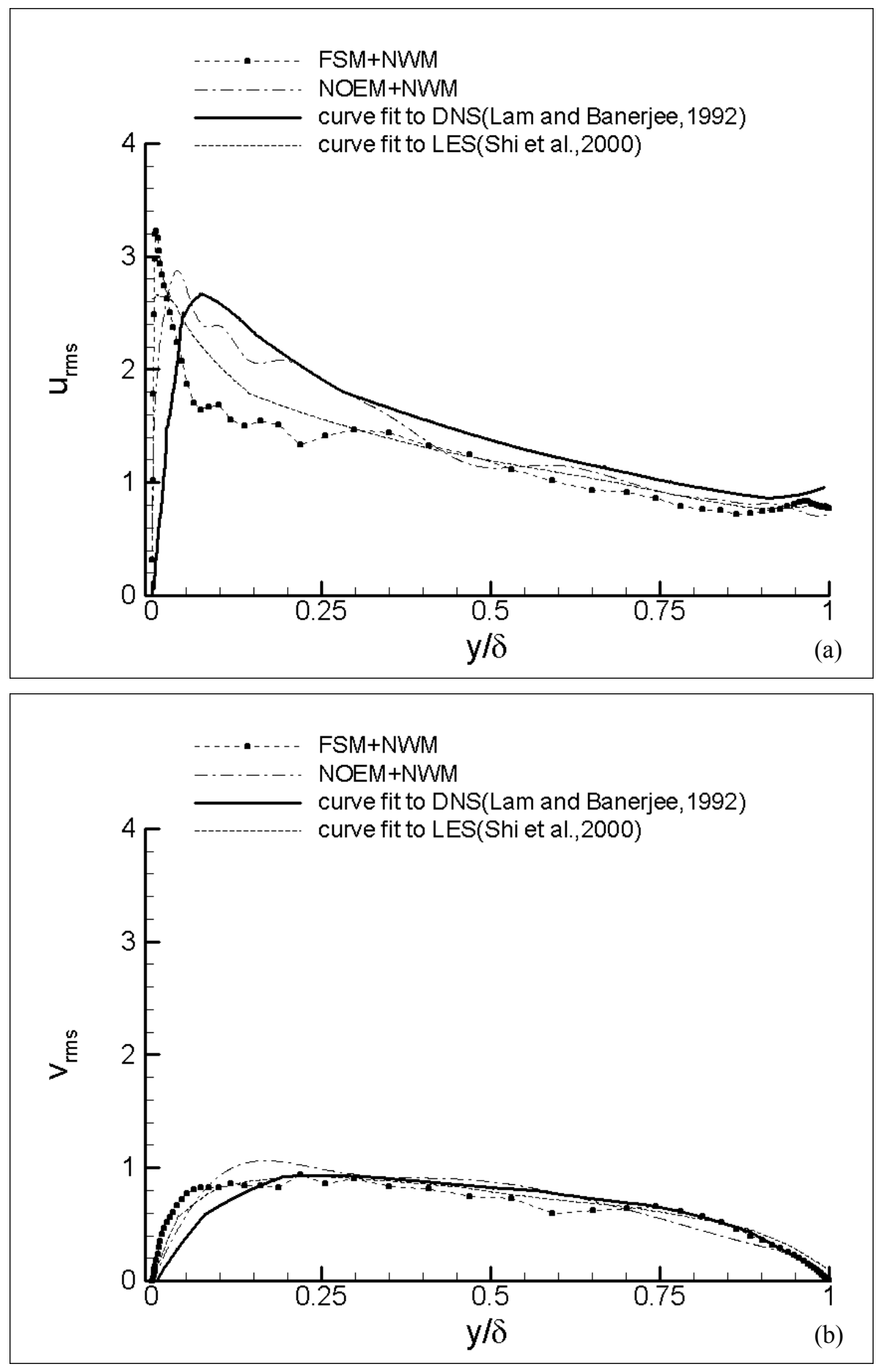

Figure 3.30. Vertical distributions of turbulence intensities; Comparisons between the present NOEM+NWM with FSM+NWM and measurements, predictions by DNS and LES studies in a) streamwise b) vertical directions: (values are normalized with $u_{\tau}$ ) 


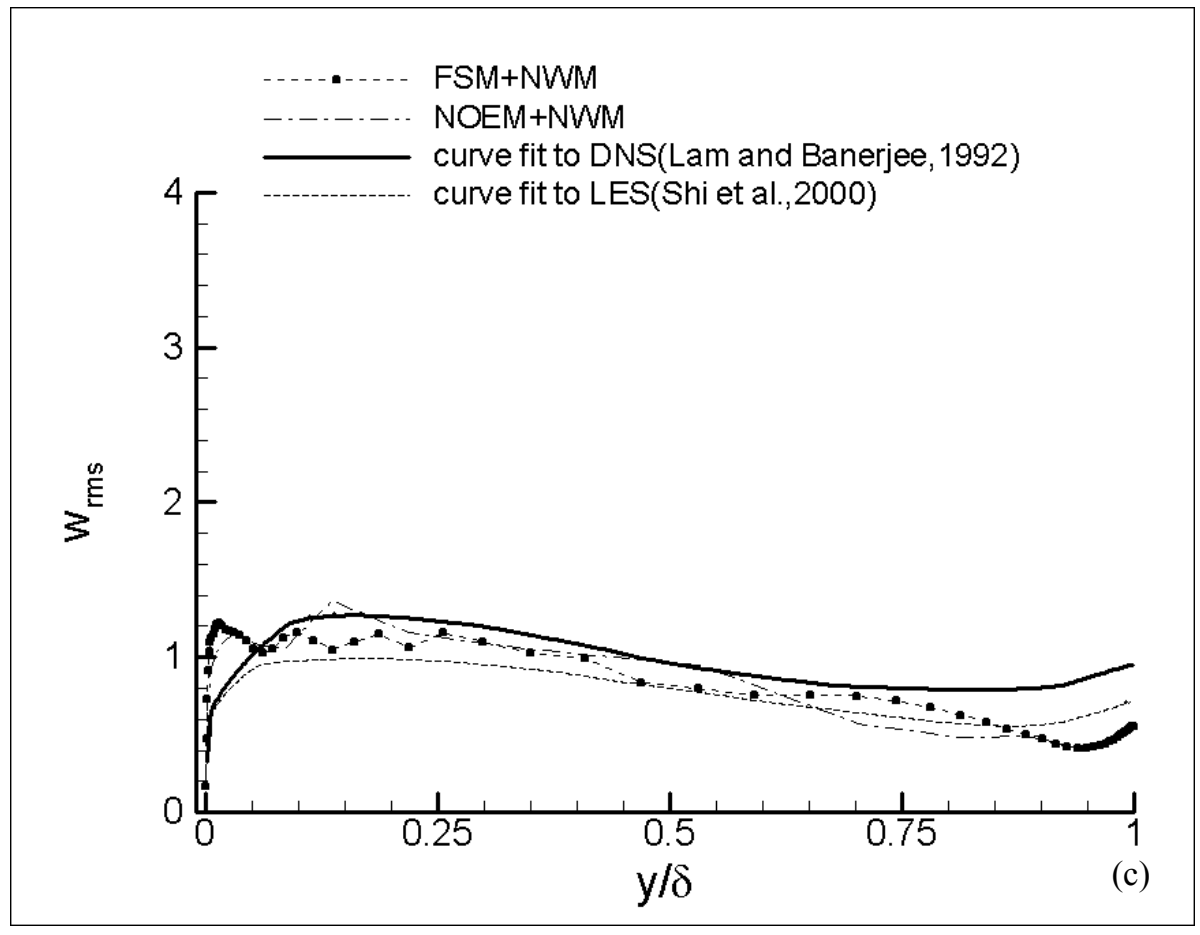

Figure 3.30 c) Vertical distributions of turbulence intensities; Comparisons between the present NOEM+NWM with FSM+NWM and measurements, predictions by DNS and LES studies in spanwise directions: (values are normalized with $\mathrm{u}_{\tau}$ ) 


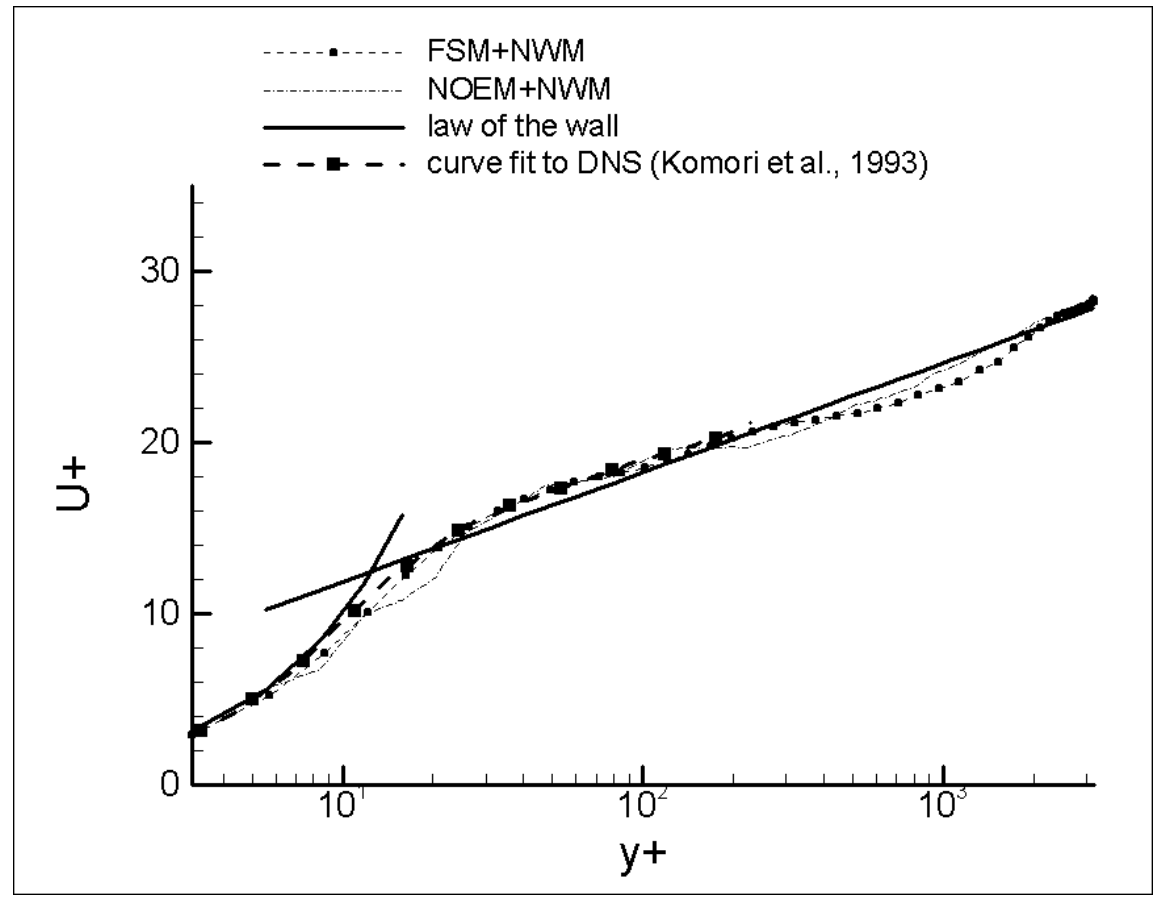

Figure 3.31. Comparison of the mean flow velocity profile 
Lam and Banerjee (1992) is observed until $y / \delta<0.4$. It should be noted that the influence of the non-linear one equation model has been included only through the eddy viscosity modification, which is essentially post processing. However, as seen from the results, even with such a modification the model produces anisotropic components of resolved velocity fluctuations. The spanwise and streamwise turbulence intensities increase whereas the vertical turbulence intensities decrease near the free surface, as expected. The time averaged velocity distributions normalized by friction velocity, $\mathrm{u}_{\tau}$, for the open channel flow are plotted against the dimensionless wall unit y+ in Figure 3.31. Results from the case using NOEM+NWM follow the logarithmic distribution slightly better than the prediction from the case with FSM+NWM, especially near the free surface region.

These results indicate that very reasonable turbulence statistics are predicted using FSM + NWM and NOEM+NWM and both can be used as a SGS model in the simulation of the ship wake turbulence. The results also indicate that NOEM+NWM results seem to be in better agreement with the log-law near the free surface compared to those from FSM+NWM. However it should be kept in mind that both models may underpredict the turbulent intensities of $\mathrm{u}^{\prime}, \mathrm{w}^{\prime}$ at the free surface that is believed to be due to grid resolution. 


\subsubsection{Application of FSM to Ship Wake}

First, FSM has been used as a SGS model in the turning ship wake calculations using a fine grid. The results have been compared with the results obtained from the standard Smagorinsky model. When the unsteady velocity fluctuations are compared, the approximate resolved frequency of the velocity fluctuations is found to be around $24 \mathrm{~Hz}$ in all three directions with standard Smagorinsky model and $30 \mathrm{~Hz}$ with FSM as seen in Figure 3.32. This shows that slightly higher frequencies and smaller turbulence scales are captured with the inclusion of the free surface effects. From Figure 3.33 and Figure 3.34, it looks like the Smagorinsky model predicts slightly higher turbulent viscosity which causes the structures to diffuse somewhat more when compared to that obtained from with FSM. From Figure 3.35 to Figure 3.38, for both models, it can be stated that big vortices in the IDP become somewhat weaker, however they do not disappear. LES captures most of the vortices. Figure 3.39 shows the mean velocity profiles at 3 vertical locations at IDP (Hyman, 2001), an angle of $5^{\circ}\left(\mathrm{x}^{\prime} / \mathrm{L}=0.80\right)$ without FSM and an angle of $5^{\circ}\left(x^{\prime} / L=0.80\right)$ with FSM for fine grid simulations respectively. In Figure 3.40, the mean velocity profile at 3 vertical locations at an angle of $15^{\circ}\left(\mathrm{x}^{\prime} / \mathrm{L}=1.30\right)$ without $\mathrm{FSM}$ and an angle of $15^{\circ}\left(\mathrm{x}^{\prime} / \mathrm{L}=1.30\right)$ with FSM has been shown. Moreover, Figure 3.41 shows the mean velocity profiles at 3 spanwise locations selected at IDP (Hyman, 2001), an angle of $5^{\circ}\left(x^{\prime} / L=0.80\right)$ without FSM and an angle of $5^{\circ}\left(x^{\prime} / L=0.80\right)$ with FSM for fine grid simulations respectively. In Figure 3.42, the mean velocity profile at 3 same spanwise locations at an angle of $15^{\circ}\left(\mathrm{x}^{\prime} / \mathrm{L}=1.30\right)$ without FSM and an angle of $15^{\circ}\left(\mathrm{x}^{\prime} / \mathrm{L}=1.30\right)$ with FSM has been shown. Figure 3.43 and Figure 3.44 show the root mean square velocities along the depth of the wake at angle of $5^{\circ}\left(\mathrm{x}^{\prime} / \mathrm{L}=0.80\right)$ and at angle of $15^{\circ}$ 
$\left(x^{\prime} / L=1.30\right)$ respectively. Both figures show that at the free surface, for both models, the vertical fluctuations are damped and the energy is redistributed to spanwise and streamwise directions, as expected. However, with FSM, it is observed that there is more energy redistributed to the spanwise direction.

Figures 3.45 to 3.47 present the velocity vectors at vertical cross-sections at angles of $5^{\circ}, 15^{\circ}$ and $25^{\circ}$ with the z-axis for both with and without FSM. As seen in Figure $3.45(\mathrm{a} \& \mathrm{~b})$ both models have well defined turbulent structures, however, FSM induces a slightly more diffusive (or smearing) flow field along the depth. Here, it can be seen that the vortical structures are penetrating deeper than those without FSM. In Figures $3.46(\mathrm{a} \& \mathrm{~b})$ the magnitudes of velocities using FSM are higher than those without using FSM. The case with FSM shows better defined turbulent structures. Eddies seem to be more diffusive and less energetic if FSM is not applied. The intensities of the velocity fluctuations at an angle of $25^{\circ}$ with the z-axis as seen in Figures $3.47(\mathrm{a} \& \mathrm{~b})$ are much less than those observed at the near wake for both cases. Still a significant amount of coherent structures can be captured in both cases. It is obvious that these structures are better defined if FSM is used. It can be stated from the overall trend that the structures seem to be more energetic and better defined near the free surface for the case with FSM then the case without FSM.

From the vorticity contours in Figures $3.48(\mathrm{a} \& \mathrm{~b})$, it is observed that the highest and lowest vorticity magnitudes are more pronounced in the case with FSM, especially in the near wake and the middle wake region. In the far wake region, both cases show decay due to the coarser grid resolution. It should be pointed out that the turbulence observed is asymmetric across the wake and there is a sharp edge on inside of the turn. 
In Figure 3.49, the resolved turbulence kinetic energy for the turning ship wake simulations without FSM using the fine grid is compared with the finest grid predictions and those of a non-turning ship. The locations for both studies are taken to be at the highest kinetic energy value obtained from the IDP (along the centerline for the non turning ship and at $\mathrm{y}=-0.1 \mathrm{E}-02$ and $\mathrm{z}=-3.18$ for the turning ship wake simulation). The resolved TKE from the simulations using the finest grid is higher than that from the simulations using the fine grid as expected. When compared to the predictions of a nonturning ship wake, both of the present calculations for the turning shi case indicate less kinetic energy values. However, it should be noted that, this may be due to the coarser grid resolution in the far wake. Overall the trends are similar, but there is a sinusoidallike distribution of the TKE prediction in the near wake for the straight ship case. It may be because of the existing surface wave from the RANS calculations (Stern and Wilson, 2000). This indicates that some wave information may be present implicitly in the inflow boundary. However, this sinusoidal-like distribution of TKE is not seen in the simulations of a ship on a circular track, which may imply that there isn't any wave information present in the RANS simulations (Hyman, 2001). It is commonly seen that bubbles will travel towards the end of the calculation domain for all of these simulations.

In Figure 3.50, the resolved turbulence kinetic energy for the turning ship wake using fine grid with and without FSM has been analyzed. Here, both simulations predict that, the kinetic energy has a similar trend. In the near wake and through the calculation domain, predictions using FSM seems to have slightly higher kinetic energy values, however, in the far wake, both decrease and their values are almost the same. 

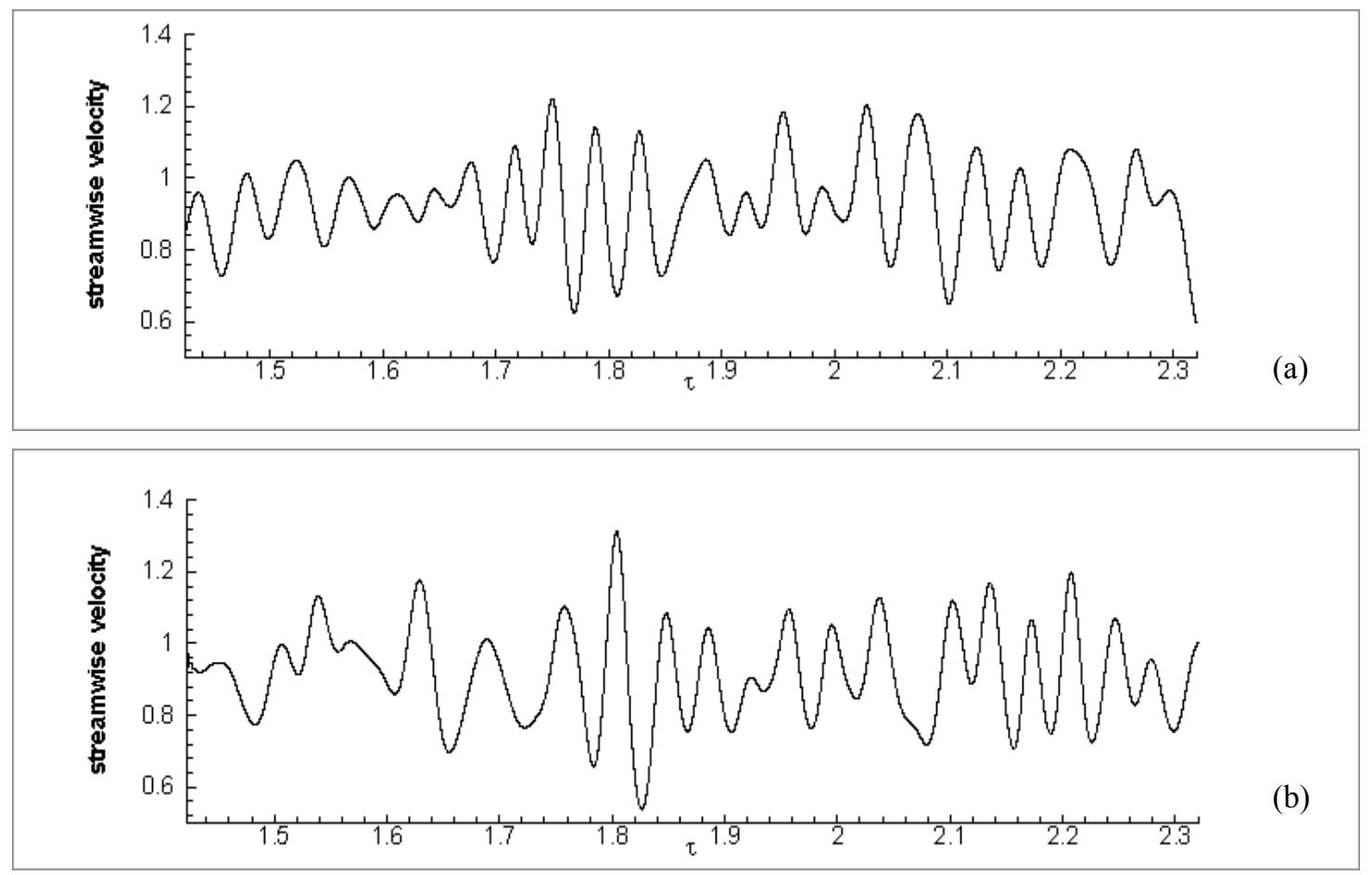

Figure 3.32. Temporal history of streamwise velocity components at $10^{\circ}(x=1.02 y=-$ $0.001 \mathrm{z}=-3.154881$ ) (fine grid) a) without b) with FSM 

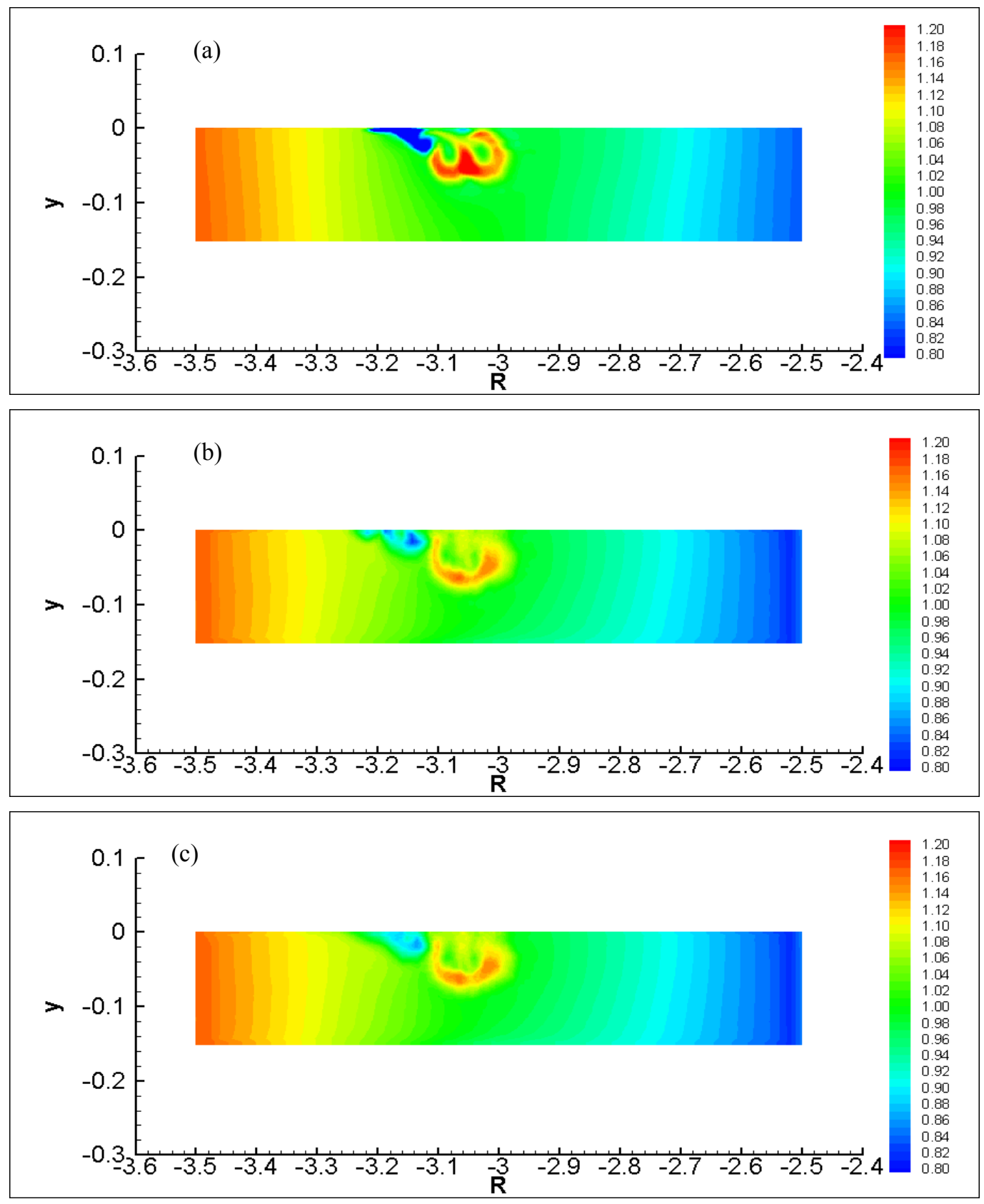

Figure 3.33. The mean axial velocity color contours at a) IDP (Hyman, 2001) b) an angle of $5^{\circ}\left(\mathrm{x}^{\prime} / \mathrm{L}=0.80\right)$ without FSM b) an angle of $5^{\circ}\left(\mathrm{x}^{\prime} / \mathrm{L}=0.80\right)$ with FSM (fine grid) 

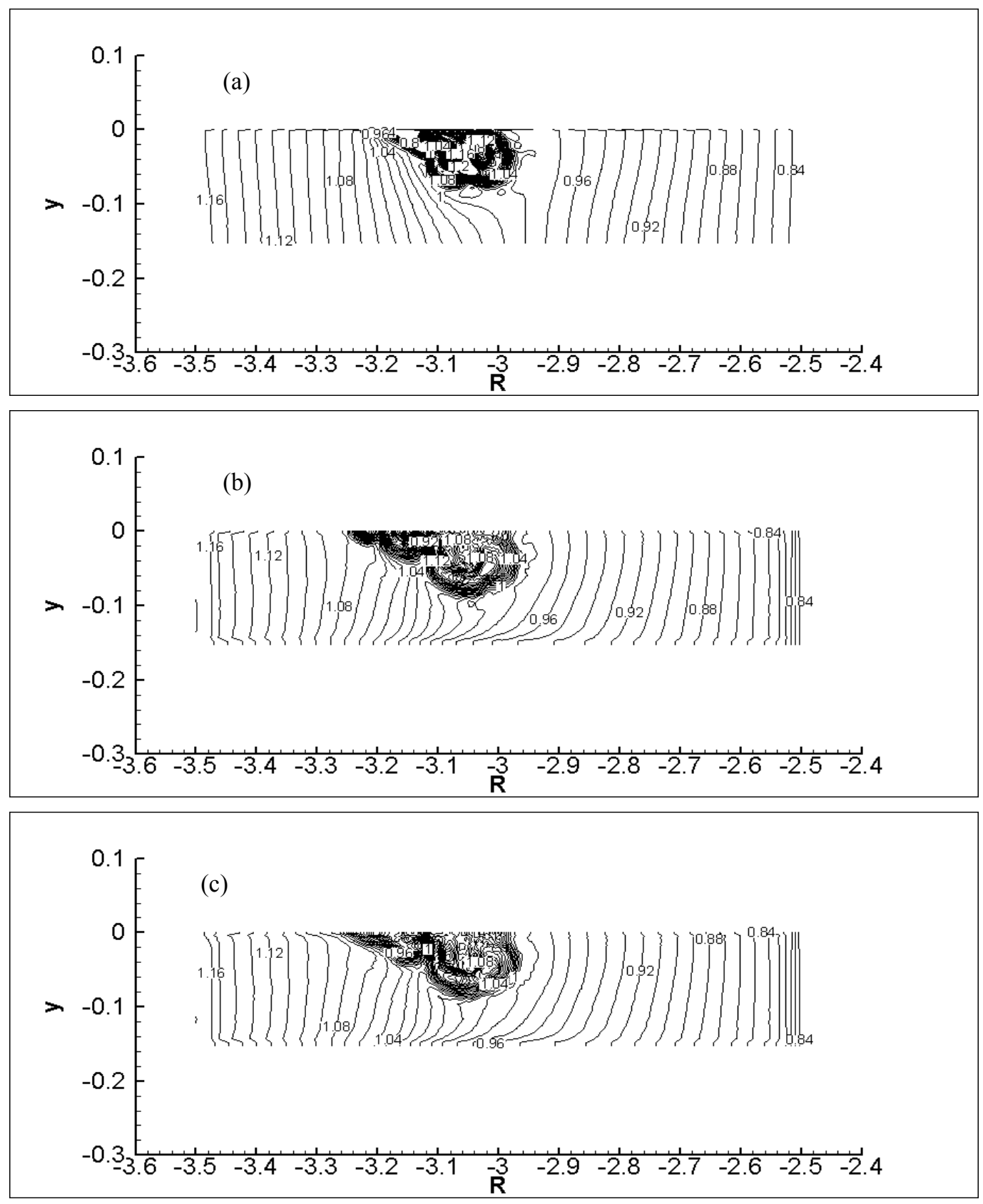

Figure 3.34. The mean axial velocity line contours at a) IDP (Hyman, 2001) b) an angle of $5^{\circ}\left(\mathrm{x}^{\prime} / \mathrm{L}=0.80\right)$ without FSM b) an angle of $5^{\circ}\left(\mathrm{x}^{\prime} / \mathrm{L}=0.80\right)$ with FSM (fine grid) 

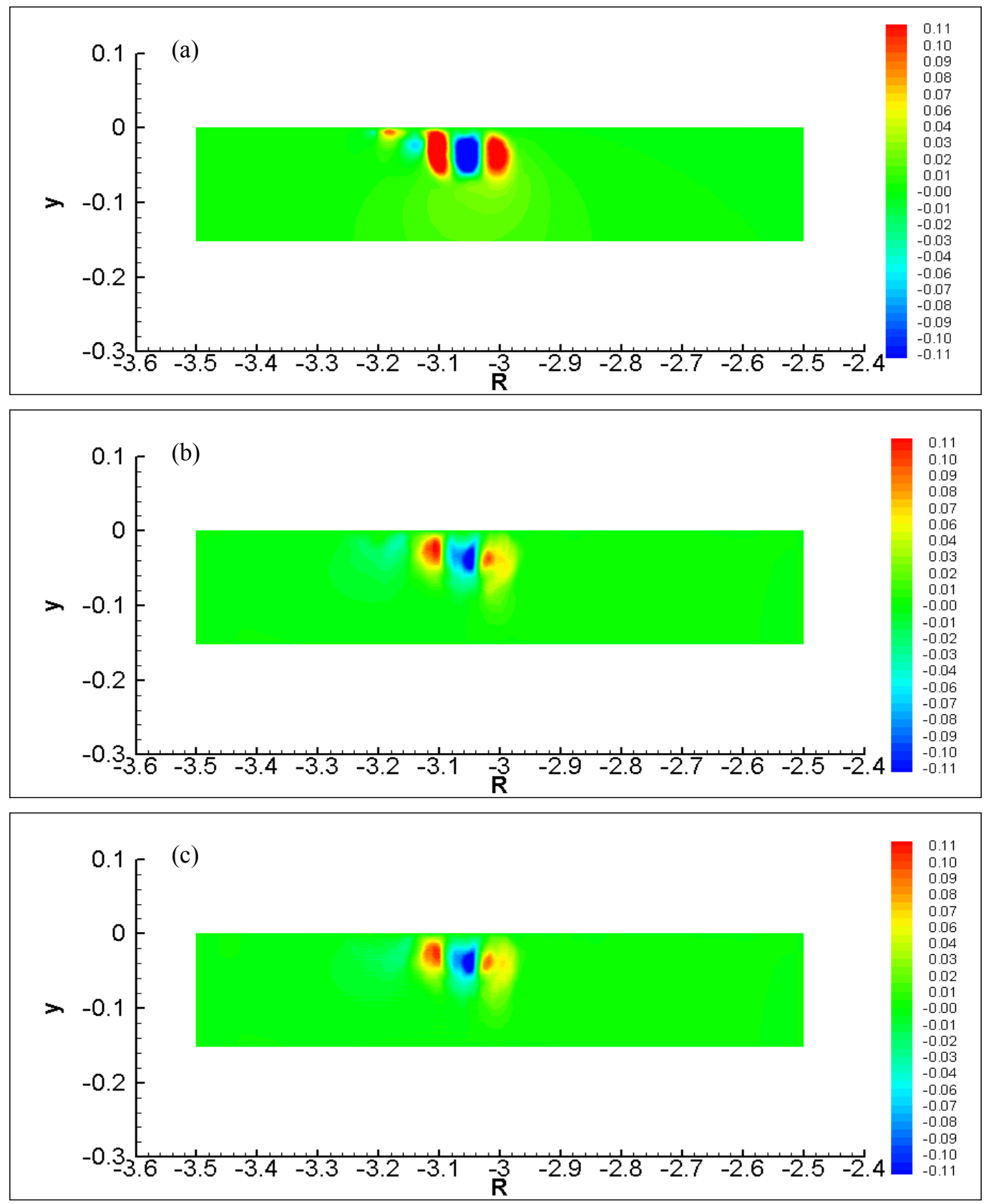

Figure 3.35. The mean vertical velocity color contours at a) IDP (Hyman, 2001) b) an angle of $5^{\circ}\left(x^{\prime} / L=0.80\right)$ without FSM $\left.b\right)$ an angle of $5^{\circ}\left(x^{\prime} / L=0.80\right)$ with FSM (fine grid) 

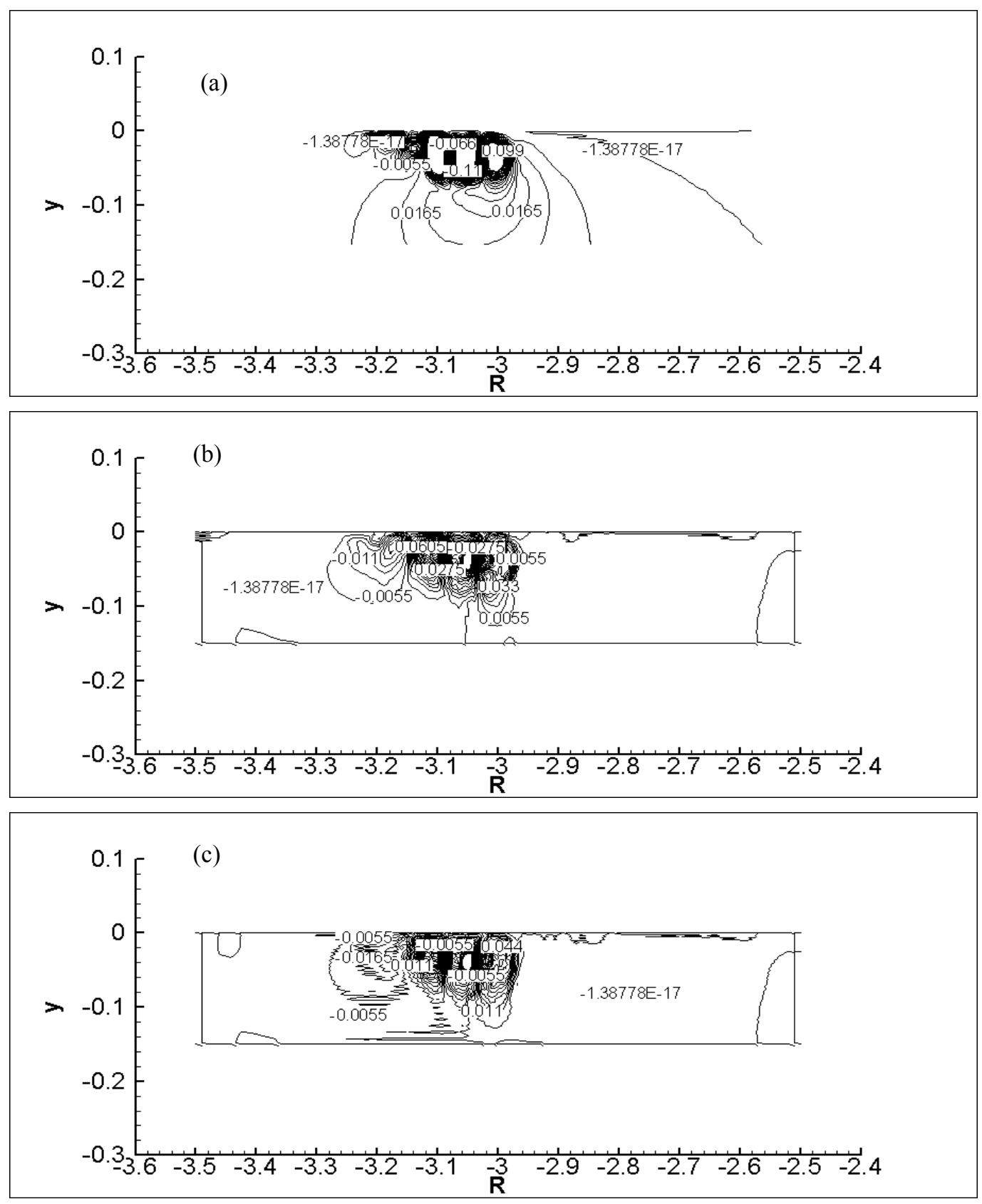

Figure 3.36. The mean vertical velocity line contours at a) IDP (Hyman, 2001) b) an angle of $5^{\circ}\left(x^{\prime} / L=0.80\right)$ without FSM $\left.b\right)$ an angle of $5^{\circ}\left(x^{\prime} / L=0.80\right)$ with FSM (fine grid) 

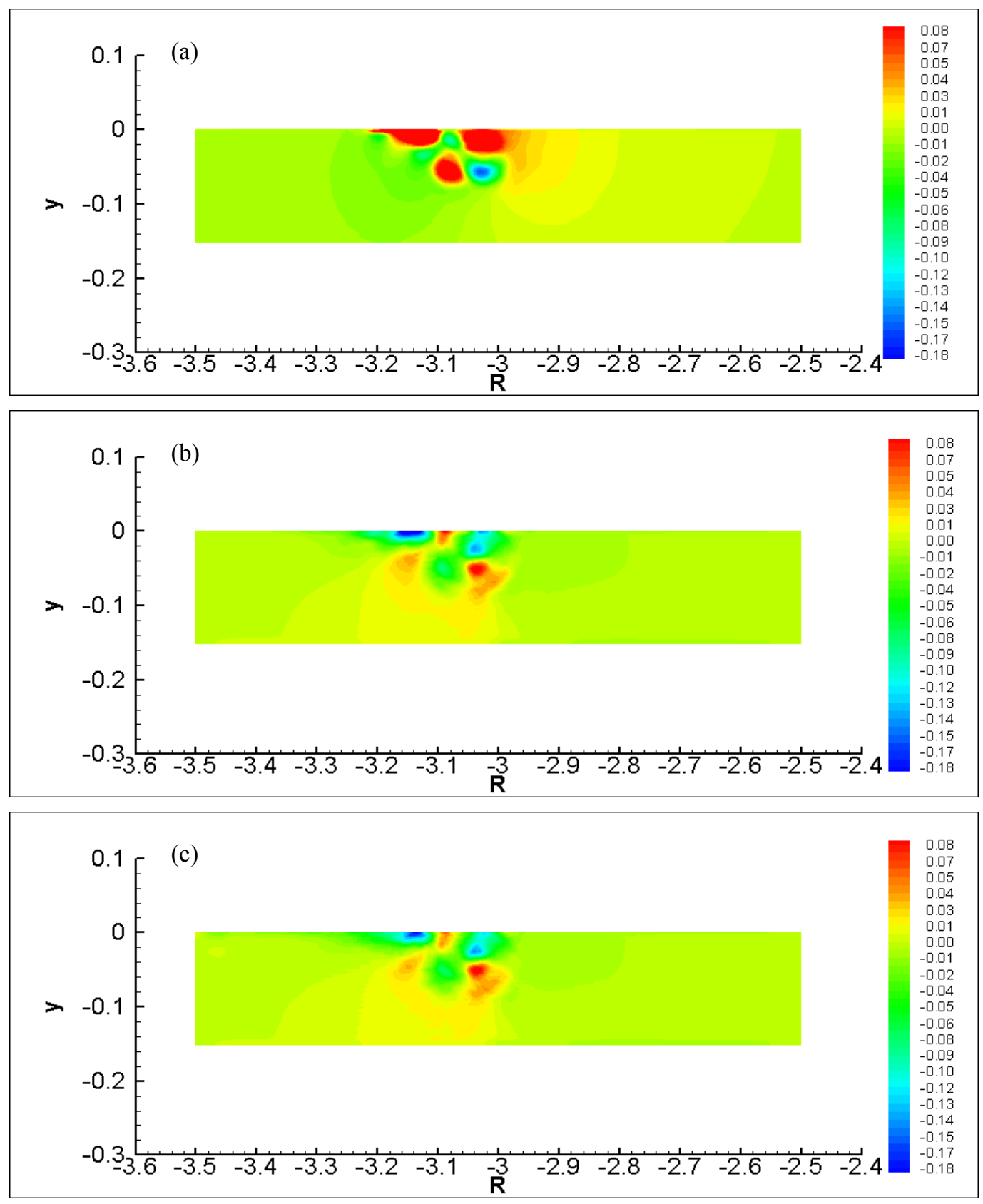

Figure 3.37. The mean transverse velocity color contours at a) IDP (Hyman, 2001) b) an angle of $5^{\circ}\left(x^{\prime} / L=0.80\right)$ without FSM $\left.b\right)$ an angle of $5^{\circ}\left(x^{\prime} / L=0.80\right)$ with FSM (fine grid) 

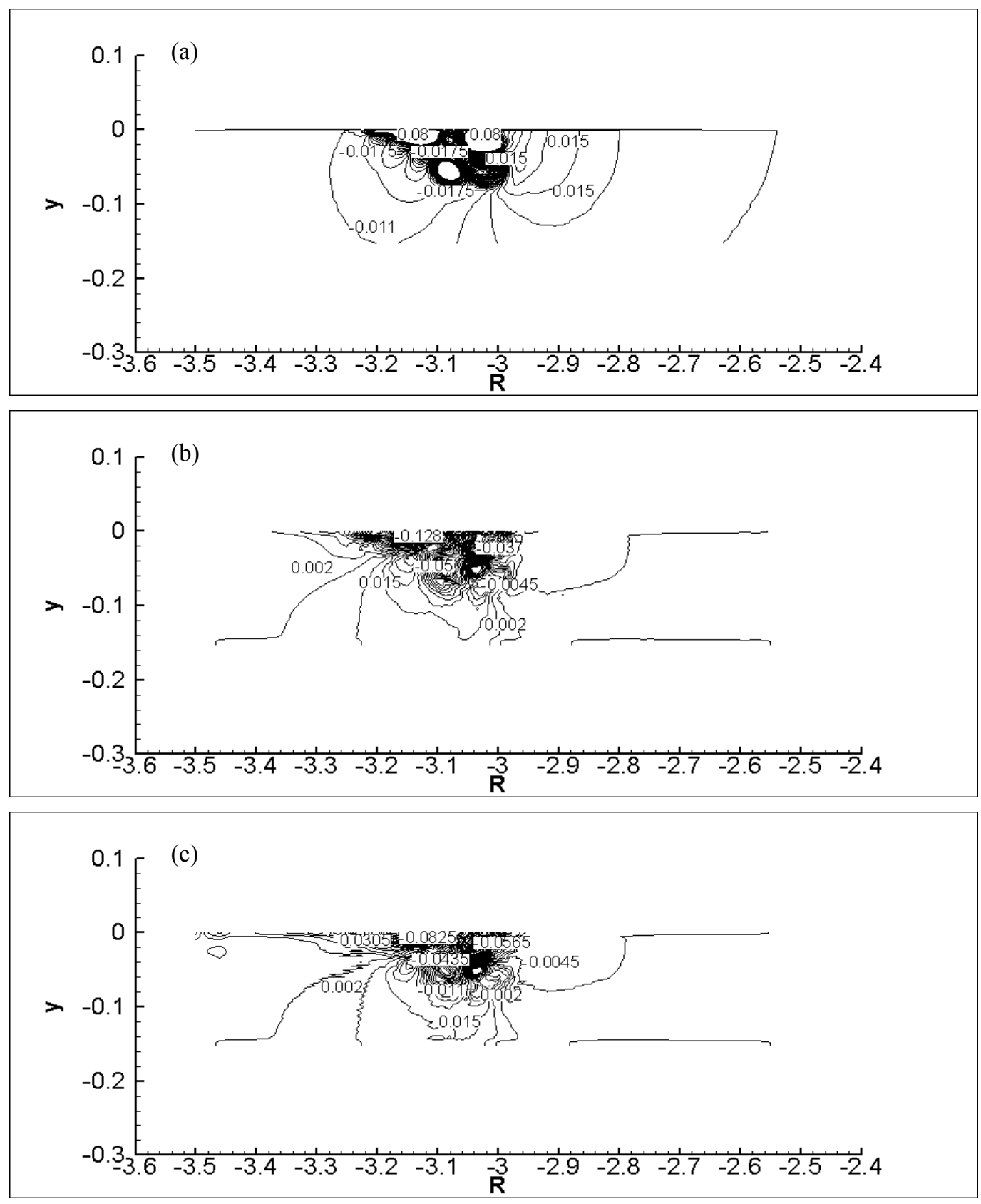

Figure 3.38. The mean transverse velocity line contours at a) IDP (Hyman, 2001) b) an angle of $5^{\circ}\left(x^{\prime} / L=0.80\right)$ without FSM $\left.b\right)$ an angle of $5^{\circ}\left(x^{\prime} / L=0.80\right)$ with FSM (fine grid) 

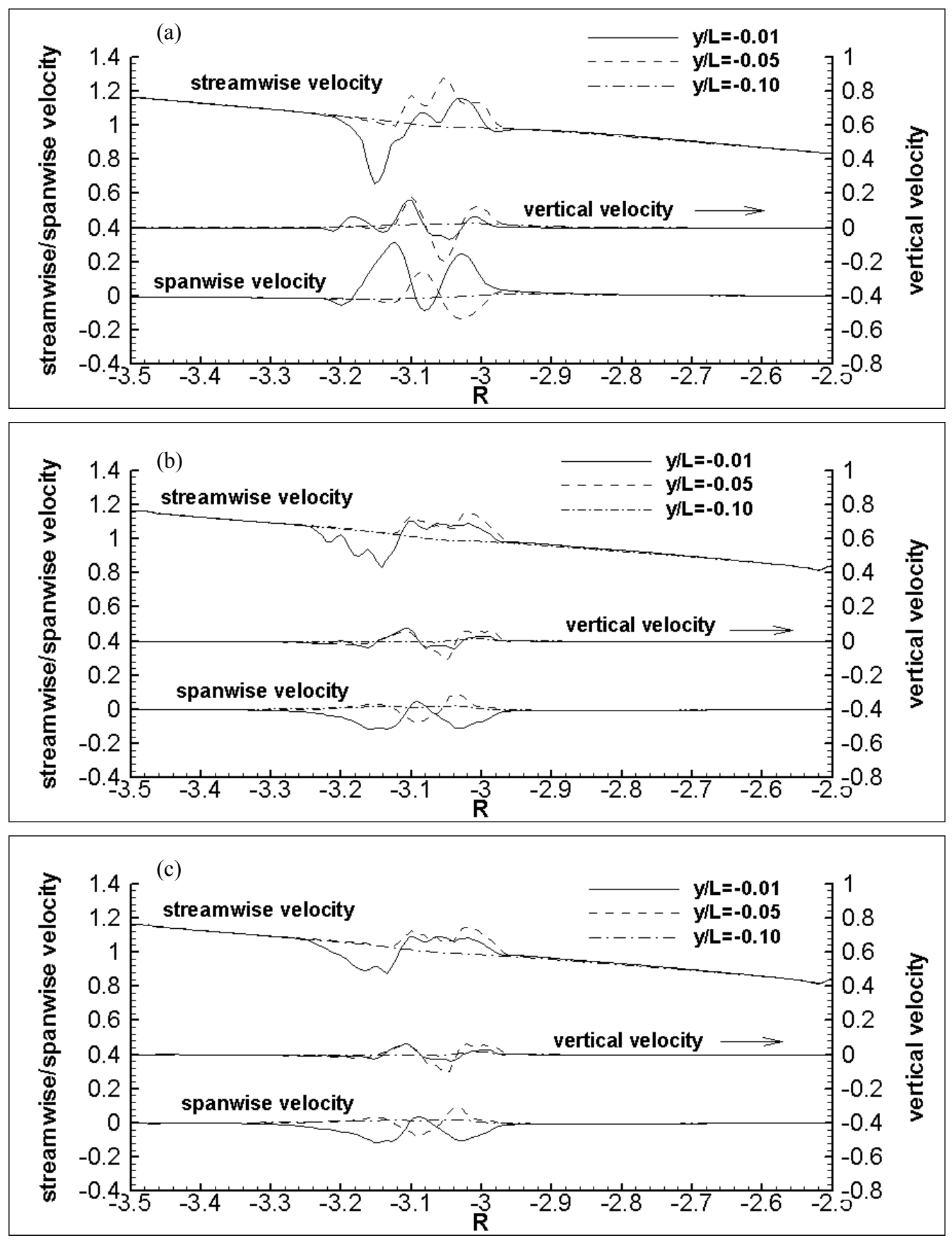

Figure 3.39. The mean velocity profiles at 3 vertical locations at a) IDP (Hyman, 2001) b) an angle of $5^{\circ}\left(x^{\prime} / L=0.80\right)$ without FSM b) an angle of $5^{\circ}\left(x^{\prime} / L=0.80\right)$ with FSM (fine grid) 

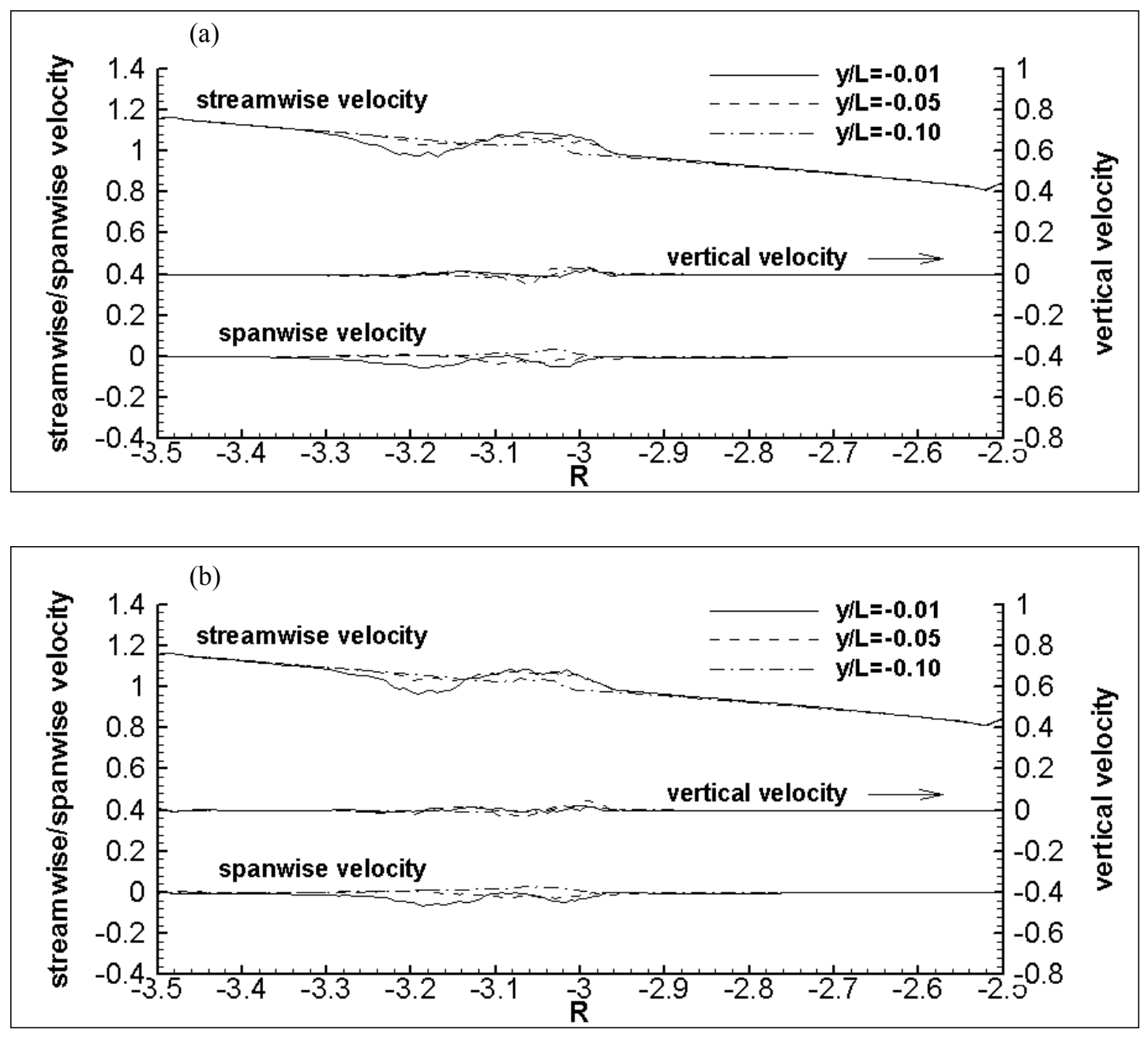

Figure 3.40. The mean velocity profiles at 3 vertical locations at a) an angle of $15^{\circ}$ $\left(\mathrm{x}^{\prime} / \mathrm{L}=1.30\right)$ without $\mathrm{FSM}$ b) an angle of $15^{\circ}\left(\mathrm{x}^{\prime} / \mathrm{L}=1.30\right)$ with FSM (fine grid) 

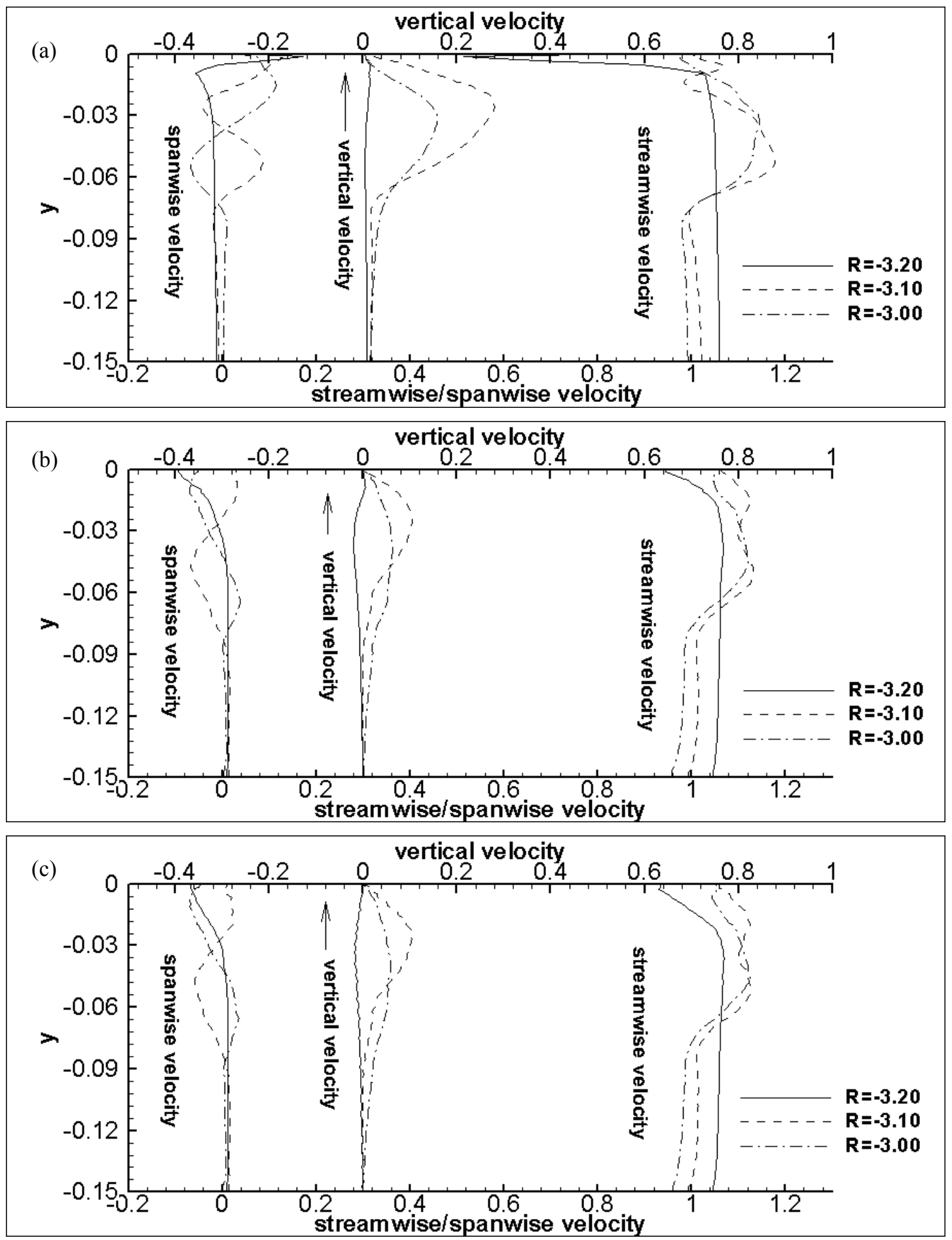

Figure 3.41. The mean velocity profiles at 3 spanwise locations at a) IDP (Hyman, 2001) b) an angle of $5^{\circ}\left(x^{\prime} / L=0.80\right)$ without FSM b) an angle of $5^{\circ}\left(x^{\prime} / L=0.80\right)$ with FSM (fine grid) 

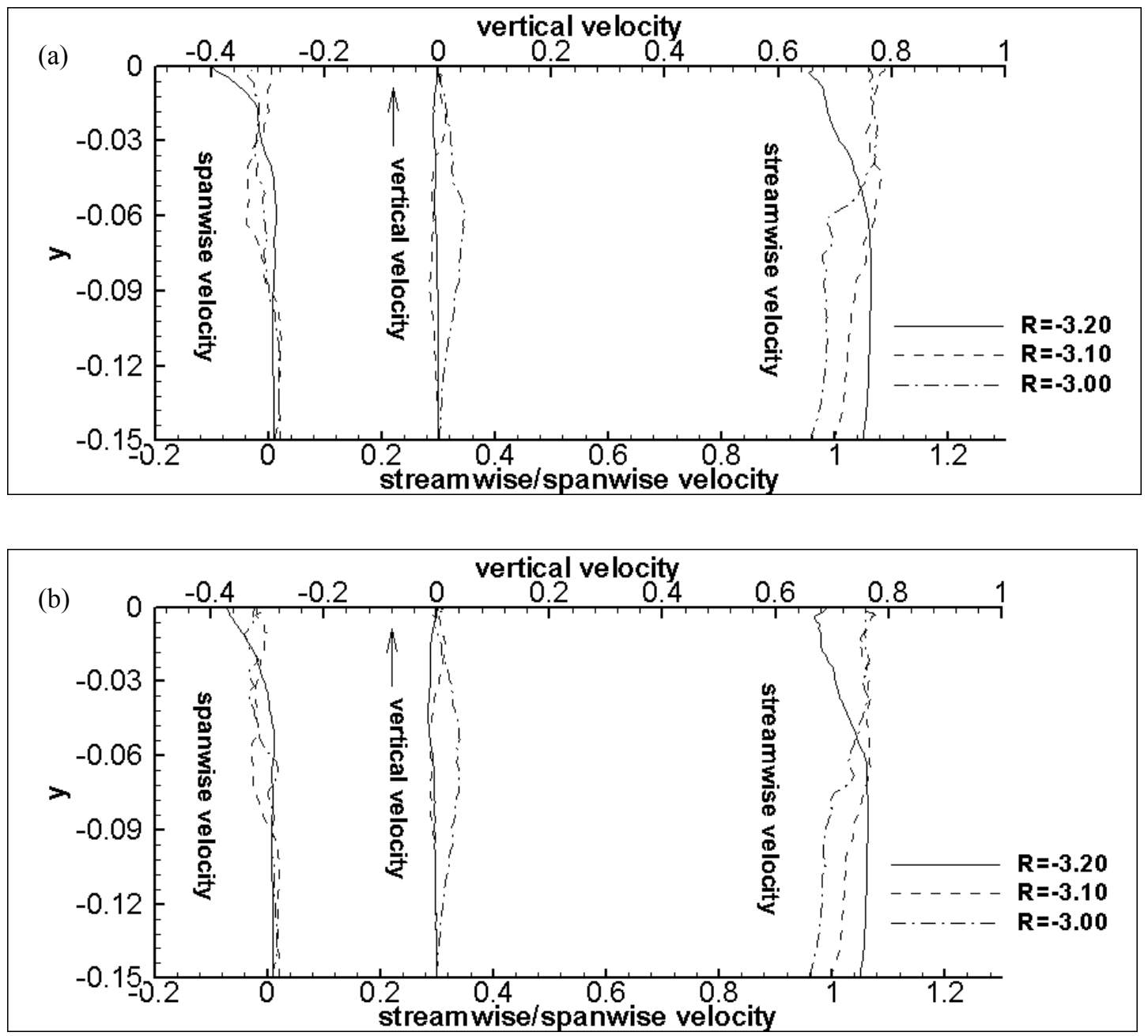

Figure 3.42 . The mean velocity profiles at 3 spanwise locations at a) an angle of $15^{\circ}$ $\left(x^{\prime} / L=1.30\right)$ without FSM $\left.b\right)$ an angle of $15^{\circ}\left(x^{\prime} / L=1.30\right)$ with FSM (fine grid) 

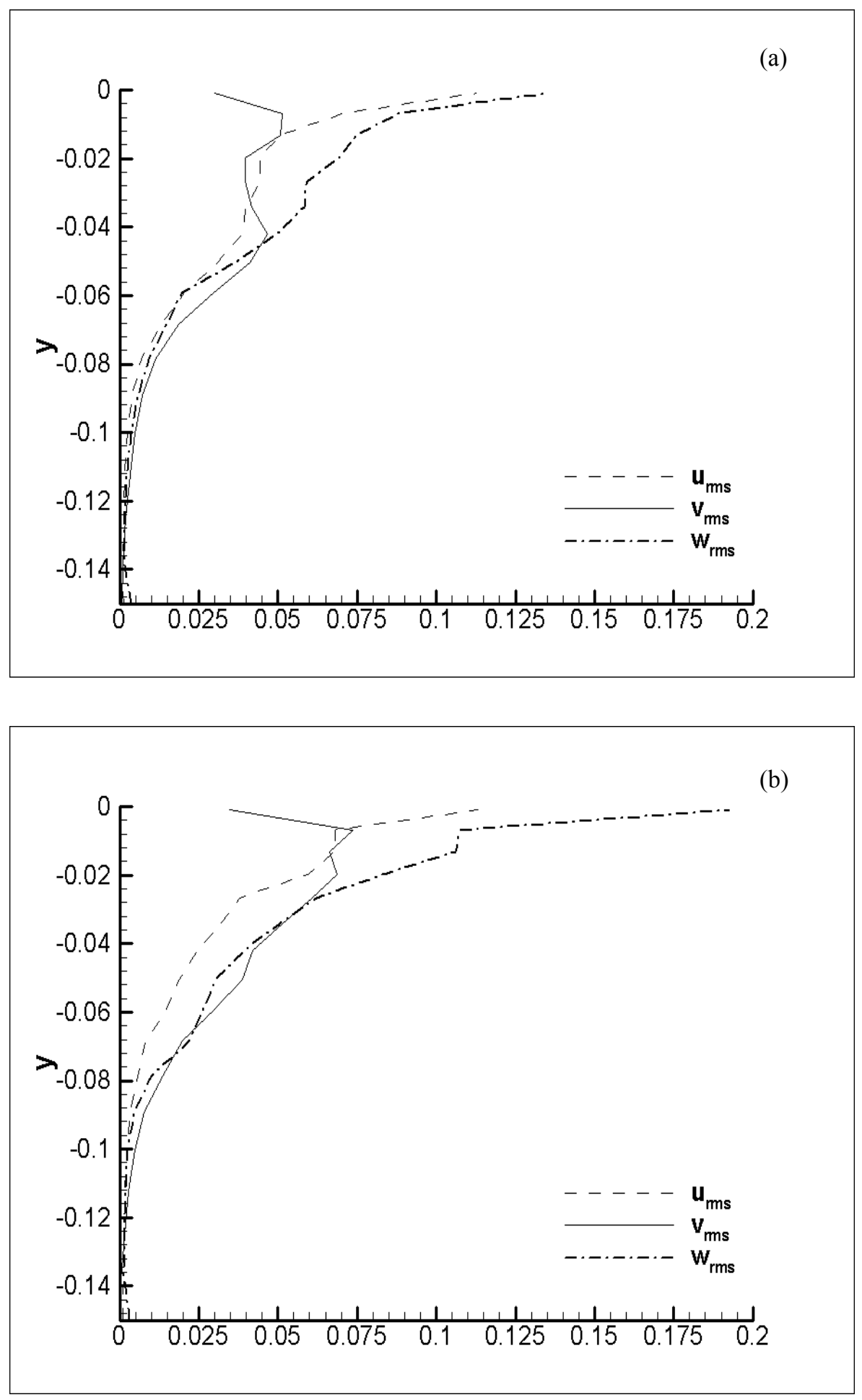

Figure 3.43. The variation root mean square velocities with depth at an angle of $5^{\circ}$ $\left(\mathrm{x}^{\prime} / \mathrm{L}=0.80\right)$ a) without FSM b) with FSM (fine grid) 

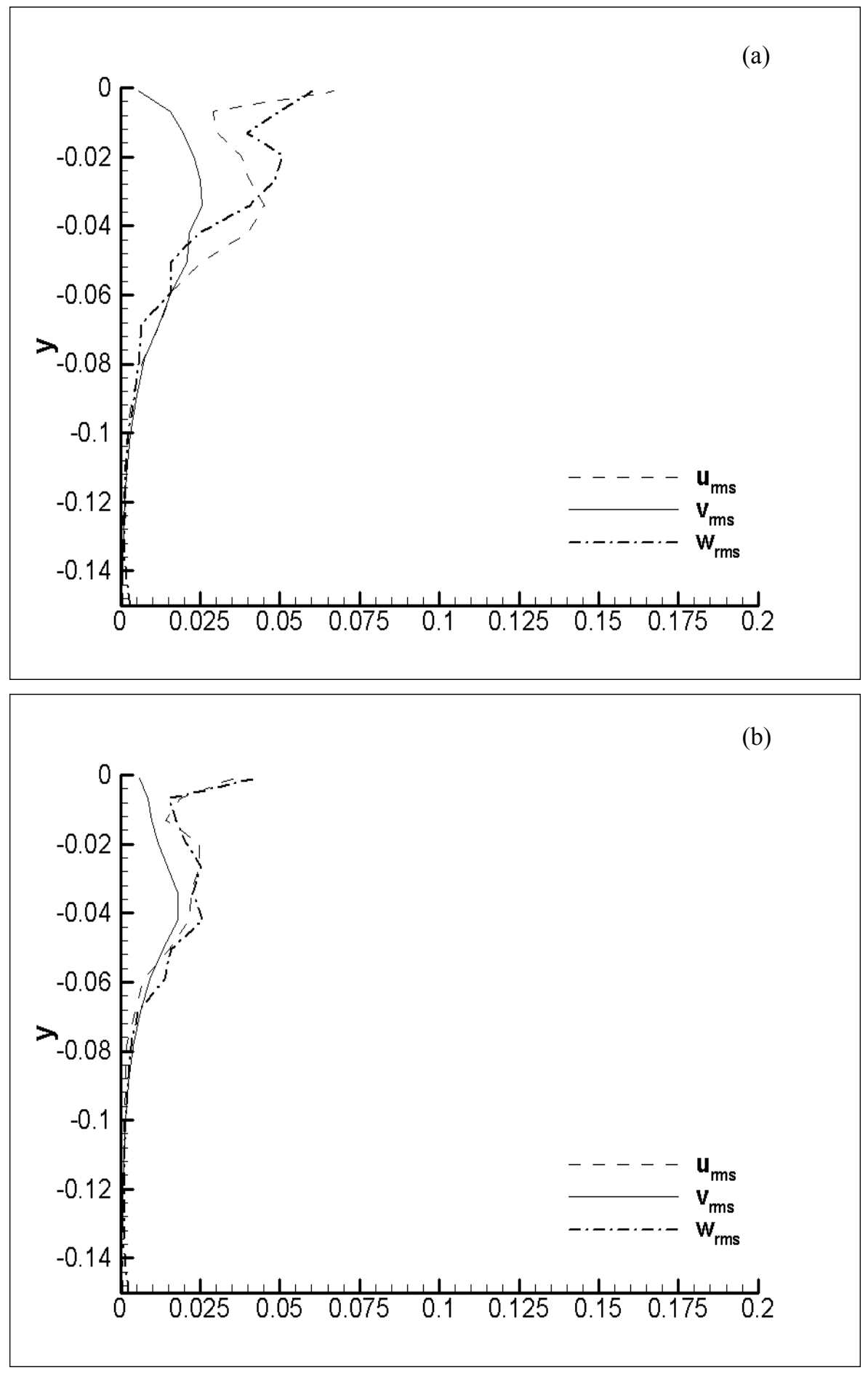

Figure 3.44. The variation root mean square velocities with depth at an angle of $15^{\circ}$ $\left(x^{\prime} / L=1.30\right)$ a) without FSM b) with FSM (fine grid) 

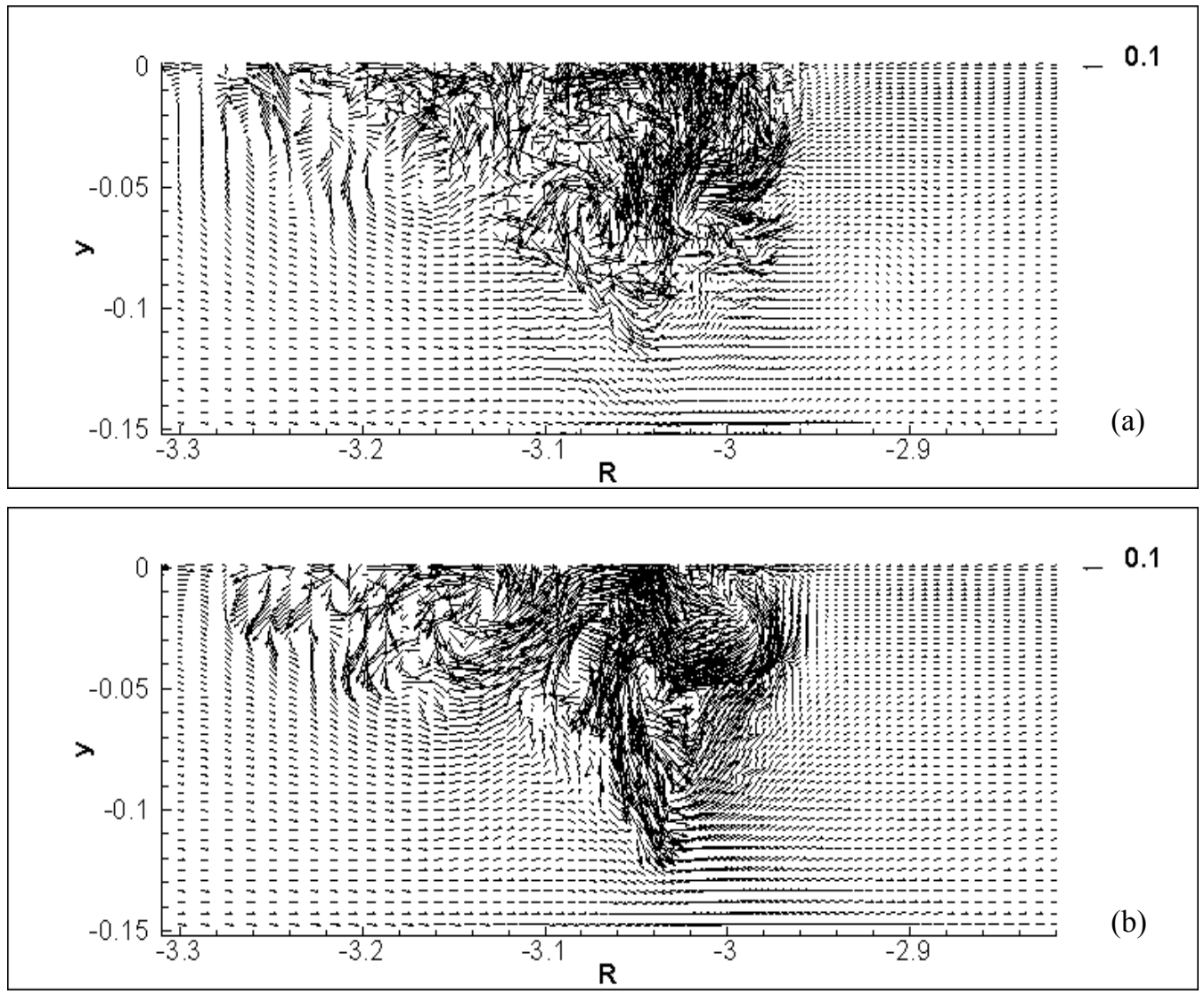

Figure 3.45 . The velocity vectors on a vertical plane at an angle of $5^{\circ}$ with the z-axis: a) without and b) with FSM (fine grid) 

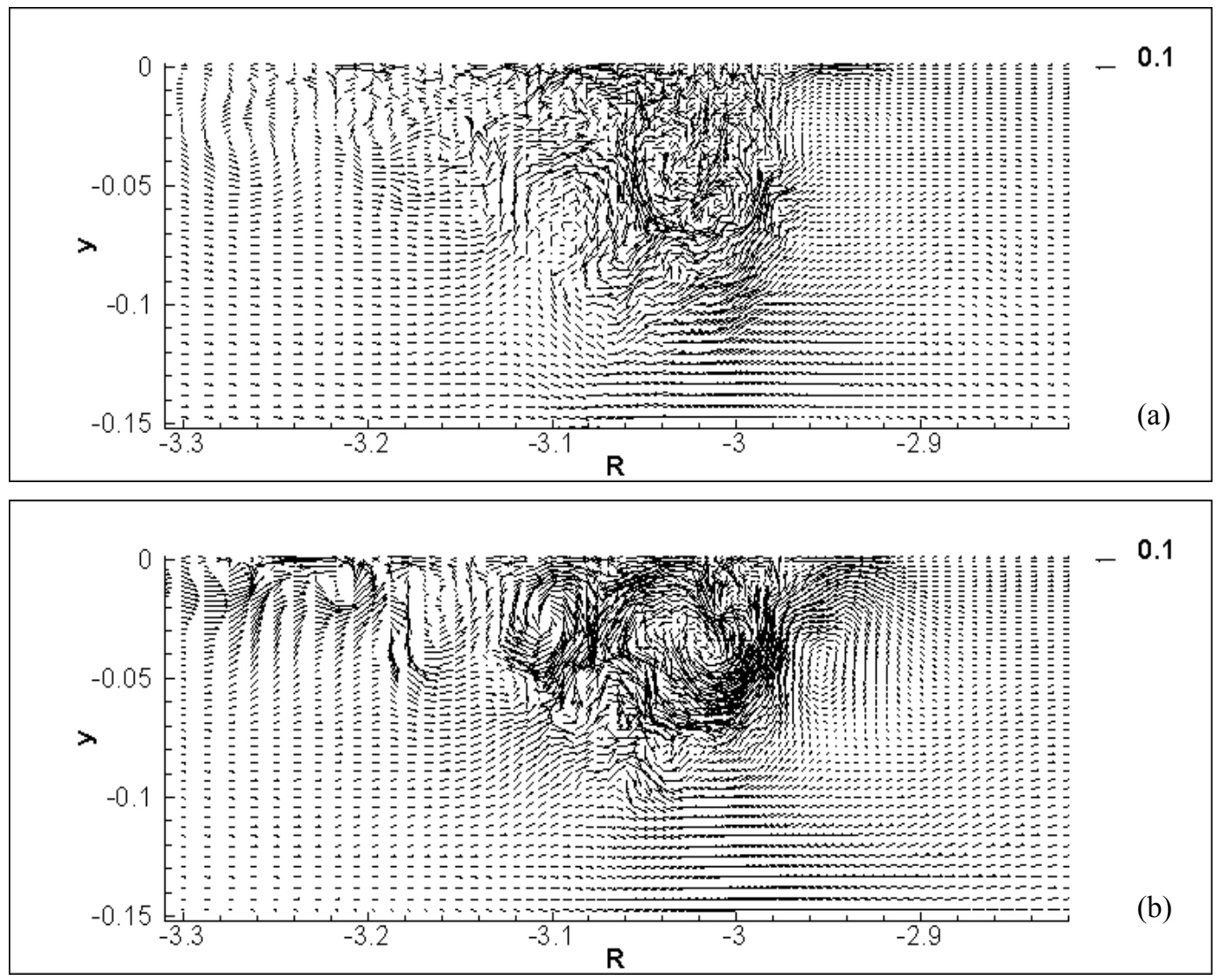

Figure 3.46. The velocity vectors on a vertical plane at an angle of $15^{\circ}$ with the z-axis: a) without and b) with FSM (fine grid) 

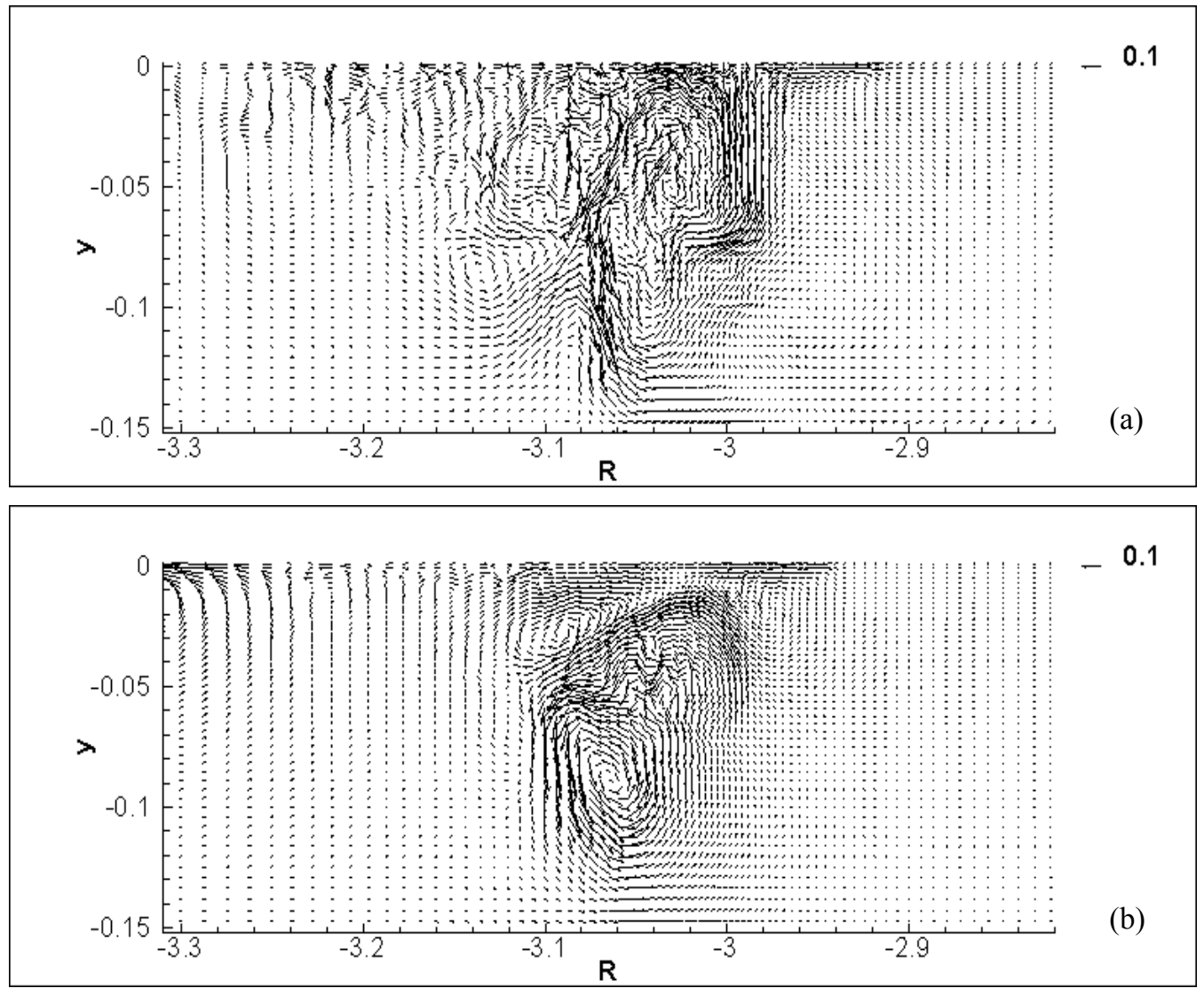

Figure 3.47 . The velocity vectors on a vertical plane at an angle of $25^{\circ}$ with the z-axis: a) without and b) with FSM (fine grid) 

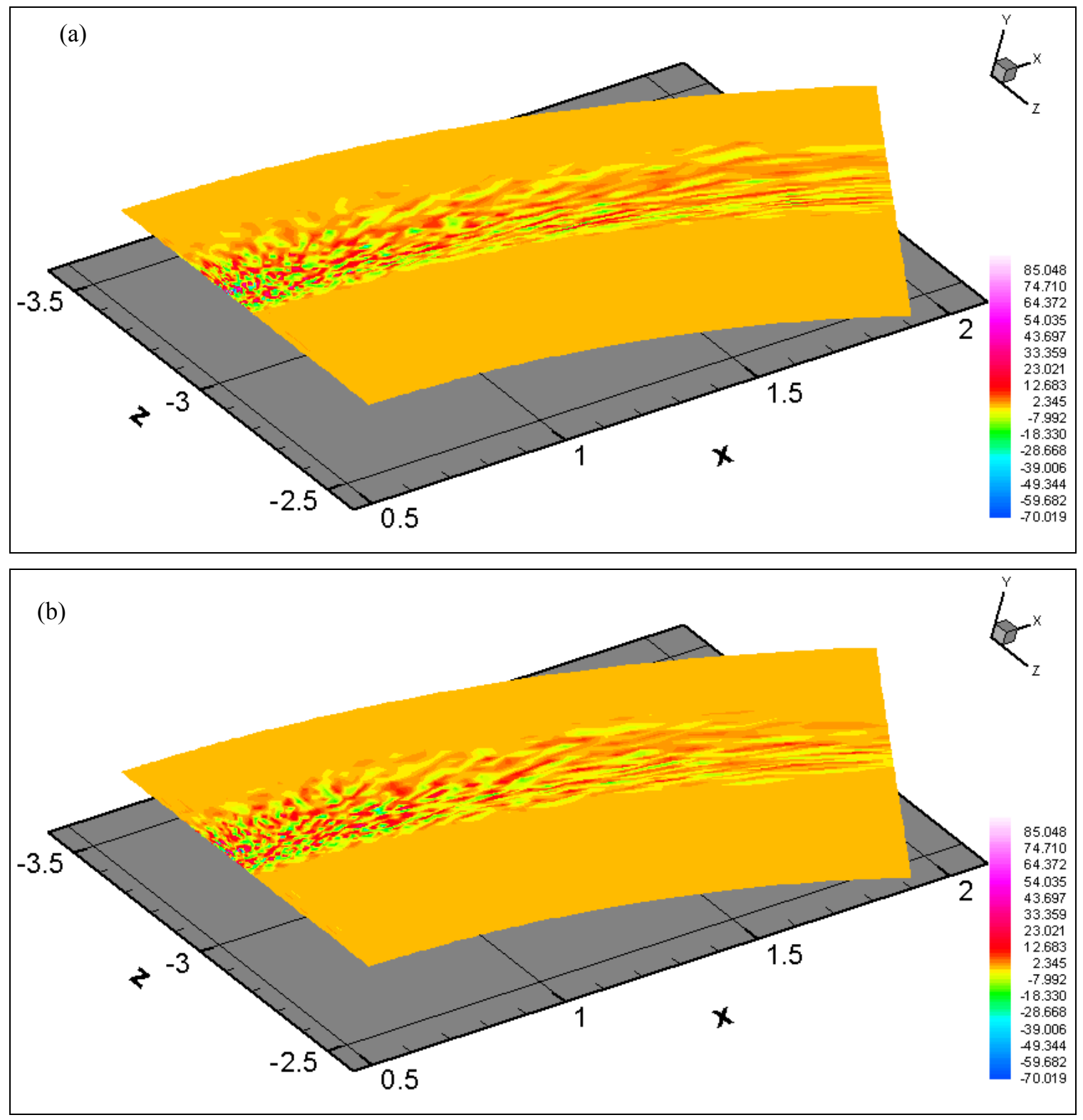

Figure 3.48. The predicted vertical vorticity, $\omega_{y}$, contours at $y=-0.1 E-02$ in the ship wake: a) without and b) with FSM (fine grid) 


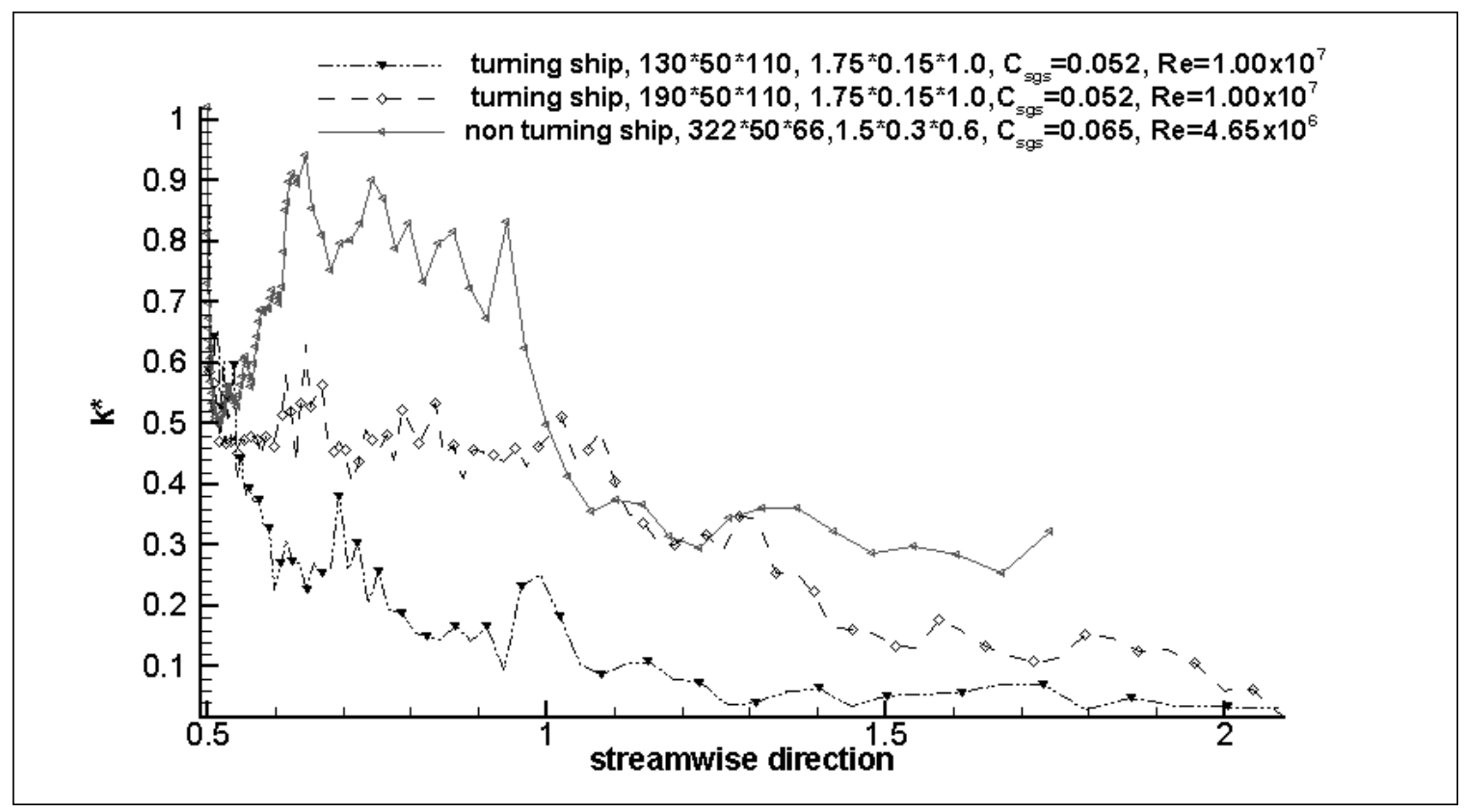

Figure 3.49. The comparison of the resolved turbulence kinetic energy normalized w.r.t its inlet value, $\mathrm{k}_{\text {inlet }}=2.5 \mathrm{e}-02$ (at $\mathbf{y}=\mathbf{- 0 . 1 E - 0 2}$ and $\left.\mathbf{z}=-\mathbf{3 . 1 8}\right)$ (Turning ship), $\mathrm{Re}=1.0 \times 10^{7}$ $\mathrm{k}_{\text {inlet }}=3.3 \mathrm{e}-03$ (at the centerline) (Non-turning ship), $\mathrm{Re}=4.65 \times 10^{6}$ for different grid resolution without FSM 


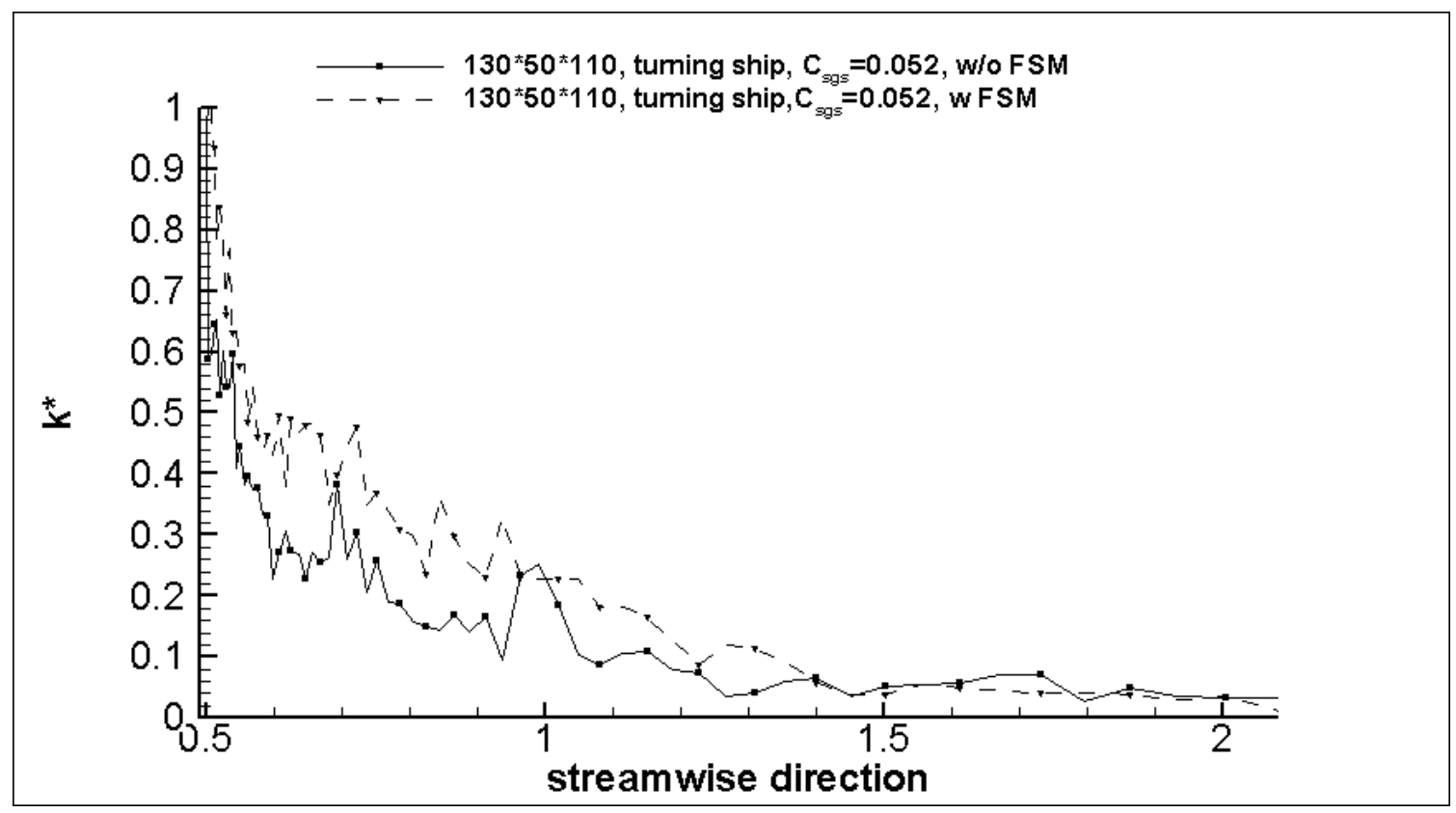

Figure 3.50. The comparison of the resolved turbulence kinetic energy normalized w.r.t its inlet value, $\mathrm{k}_{\text {inlet }}=2.5 \mathrm{e}-02$ (Turning ship), $\mathrm{Re}=1.0 \times 10^{7}$ with and without FSM (at $\mathrm{y}=$ $0.1 \mathrm{E}-02$ and $\mathrm{z}=-3.18)$ (fine grid) 
The investigation of the effect of the free surface modeling of SGS via damping of length scales has indicated that it should be included in LES to obtain better turbulence statistics in the vicinity of free surfaces such as all present in ship wake calculations.

\subsection{Uncertainty in the present LES}

Since the use of Large Eddy Simulations in engineering applications has rapidly increased, it has become necessary for the quality measurement of the assessment of the simulations claimed to be LES. Uncertainty/quality assessment in RANS (Reynolds Averaged Navier-Stokes) simulations has been investigated at length (Celik et al., 1993; Roache, 1998; Stern et al., 2001) and there are even some guidelines (Roache et al., 1986; Freitas, 1993) for assessment and reporting of numerical uncertainty in such simulations. The quality of LES depends on how accurate the SGS models are, however the assessment of uncertainty in LES is not so trivial, as both the numerical discretization error and the sub-grid scale contributions are proportional to grid size. As it was rightly pointed out by Speziale (1998) a good LES is that which tends to DNS (Direct Numerical Simulations) as the grid resolution tends to the smallest, namely the Kolmogorov scales. Therefore, there is no such thing as grid independent LES in theory, because a grid independent LES is essentially DNS, and the philosophy of LES loses its meaning. The advantage of LES over DNS is being that LES is much more economical, while it only requires the resolution of the most energetic eddies, which determine the essential flow dynamics and their consequences. If the main purpose of LES is to capture only the mean flow dynamics, this argument may not hold, but this would be a very limited and costly purpose. It is well known that physical phenomenon such as mixing and combustion are 
strongly dependent on intensity of turbulent fluctuations and the convection by these fluctuations that eventually exhibit themselves as turbulent diffusion. Hence in most applications of LES the prediction of turbulence is at least as important as prediction of mean flow quantities. With such a premise, it is necessary that some quality assessment measures be formulated for LES geared towards engineering applications.

Here, new quality index measures are proposed, and in particular one of these which are referred to as the Large Eddy Simulation Index of Quality (LES_IQ) is recommended for assessment of LES studies. Briefly the basic equation is presented with an example to demonstrate the feasibility of such an approach.

The recommended LES_IQ is based on Richardson's extrapolation and the formal order of accuracy of the discretization scheme. It is also postulated that the dispersion errors will be dominant and more detrimental to the quality of LES than the other types of error such as phase errors.

It seems more practical to make use of the resolved turbulent kinetic energy $\mathrm{k}^{\text {res }}$ versus the total $\mathrm{k}^{\text {tot }}=\mathrm{k}^{\text {res }}+\mathrm{k}^{\mathrm{sgs}}+\mathrm{k}^{\text {num }}$, the latter two being the contribution from the SGS model and the numerical dissipation, respectively. If one can make the assumption that $\mathrm{k}^{\text {eff_sgs }}=\left(\mathrm{k}^{\text {tot }}-\mathrm{k}^{\text {res }}\right)=\left(\mathrm{k}^{\mathrm{sgs}}+\mathrm{k}^{\text {num }}\right)$ scales with the mesh size $\mathrm{h}$, then an expression for the proposed LES_IQ that exhibits the quality of the LES can be formulated as,

$$
\text { LES_IQ } \mathrm{Q}_{\mathrm{k}}=\frac{k^{\text {res }}}{k^{\text {tot }}}=\frac{k^{\text {res }}}{k^{\text {res }}+a_{k} h^{p}}
$$


Here, $\mathrm{k}^{\text {res }}$ is the resolved kinetic energy, $\mathrm{k}^{\text {tot }}$ is the total kinetic energy, $\mathrm{k}^{\text {eff_sgs }}$ is the effective sgs kinetic energy, $h$ is the grid size and $a_{k}$ is a coefficient to be determined, $\mathrm{p}$ is the formal order of accuracy of the numerical scheme.

In the spirit of Richardson's extrapolation (see Roache, 1998), the effective SGS kinetic energy might be approximated as

$$
\begin{aligned}
& k^{\text {tot }}-k_{1}^{\text {res }}=k_{1}^{e f f}{ }^{\text {sgs }}=a_{k} h_{1}^{p} \\
& k^{\text {tot }}-k_{2}^{\text {res }}=k_{2}^{\text {eff }{ }_{-}^{\text {sgs }}}=a_{k} h_{2}^{p}
\end{aligned}
$$

where subscripts (1) and (2) denote quantities obtained on mesh (1) and mesh (2). In the present application, $\mathrm{h}=(\Delta \mathrm{x} \Delta \mathrm{y} \Delta \mathrm{z})^{1 / 3}$, where $\Delta \mathrm{x}, \Delta \mathrm{y}, \Delta \mathrm{z}$ are the grid cell lengths in $\mathrm{x}-, \mathrm{y}-, \mathrm{z}-$ directions, respectively. This relation can be used for calculating a local mesh size, if the details of the grid are known. However, in general, a global grid index parameter can be defined as,

$$
h_{e q}=\left[\frac{1}{N} \sum \Delta \forall_{i}\right]^{1 / 3}
$$

In Equations 3.4 and 3.5 it is assumed that the leading term in the truncation error series is dominant, in other words, the grid resolution is in the asymptotic range (see Roache, 1998).

Equations 3.4 and 3.5 can be solved for $\mathrm{a}_{\mathrm{k}}$ to yield, 


$$
a_{k}=\frac{1}{h_{2}^{p}}\left[\frac{k_{2}^{r e s}-k_{1}^{r e s}}{\alpha^{p}-1}\right]
$$

where $\alpha=h_{1} / h_{2}>1$ is the grid refinement (or coarsening) parameter.

Hence, the expression for LES_IQ $\mathrm{Q}_{\mathrm{k}}$ becomes,

$$
L E S_{-} I Q_{k}=\frac{k^{\text {res }}}{k^{r e s}+\frac{\left(k_{2}^{r e s}-k_{1}^{r e s}\right.}{\alpha^{p}-1}\left(\frac{h}{h_{2}}\right)^{p}}
$$

Rearranging Equation 3.8 for both grid sizes yields,

Fine Grid

$$
L E S_{-} I Q_{k}^{f}=\frac{1}{1+\left(1-\frac{k_{1}^{r e s}}{k_{2}^{r e s}}\right)\left(\alpha^{p}-1\right)^{-1}}
$$

\section{Coarse Grid}

$$
L E S_{-} I Q_{k}^{c}=\frac{1}{1+\left(\frac{k_{2}^{\text {res }}}{k_{1}^{\text {res }}}-1\right) \alpha^{p}\left(\alpha^{p}-1\right)^{-1}}
$$

An example of the application of the quality index has been presented using the LES results of ship wakes where the formal order of the numerical scheme is in $2^{\text {nd }}$ order, 
$p=2$. The calculated quality index for a simulation of the wake behind a ship cruising on a straight track with two different grid resolutions is shown in Figure 3.52. Figure 3.51 shows the predicted resolved kinetic energy values. It is seen that for the fine grid, LES_IQ ${ }_{k}$ in the near wake region is c.a. $55 \%$ compared to a c.a. $30 \%$ for the coarse grid. The quality deteriorates downstream where the grid resolution is coarser.

The LES_IQ ${ }_{\mathrm{k}}$ for the LES results of the wake behind a ship cruising on a circular track is shown in Figure 3.53. In Figure 3.49, the predicted resolved kinetic energy values are presented. The coarse grid index is c.a. $40 \%$ and the fine grid index is c.a. $70 \%$ near the wake. The low LES_IQ $\mathrm{Q}_{\mathrm{k}}$ values indicate that for both cases more grid refinement is necessary. More details can be obtained from Celik et al. (2003 a \& b). 


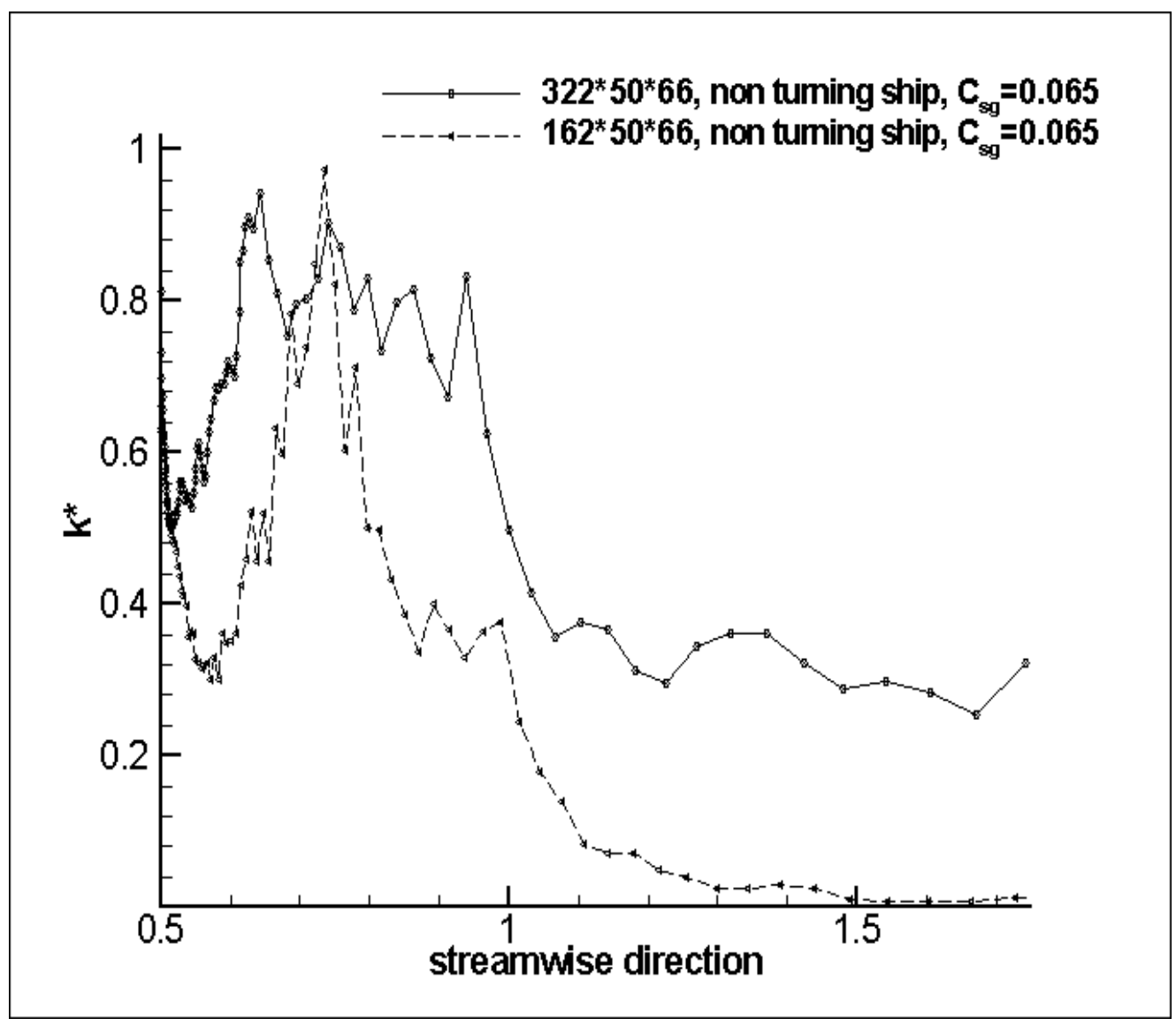

Figure 3.51. Resolved turbulence kinetic energy of the wake simulation of a ship cruising on a straight track normalized w.r.t. its inlet value $\left(\mathrm{k}_{\text {inlet }}=3.3 \mathrm{e}-03\right)$ 


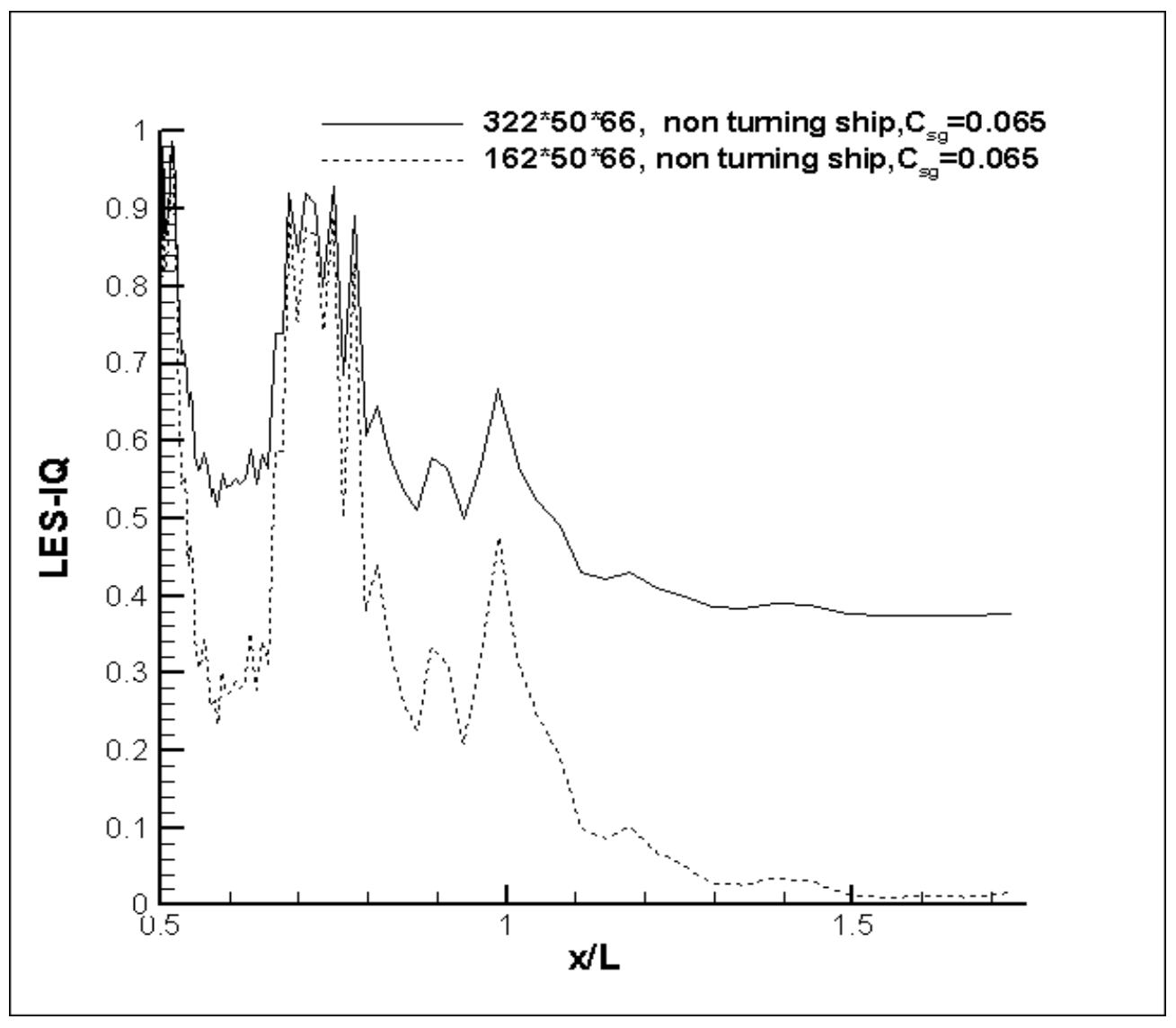

Figure 3.52. Large Eddy Simulation Index of Quality (LES_IQ) for the wake simulation of a ship cruising on a straight track 


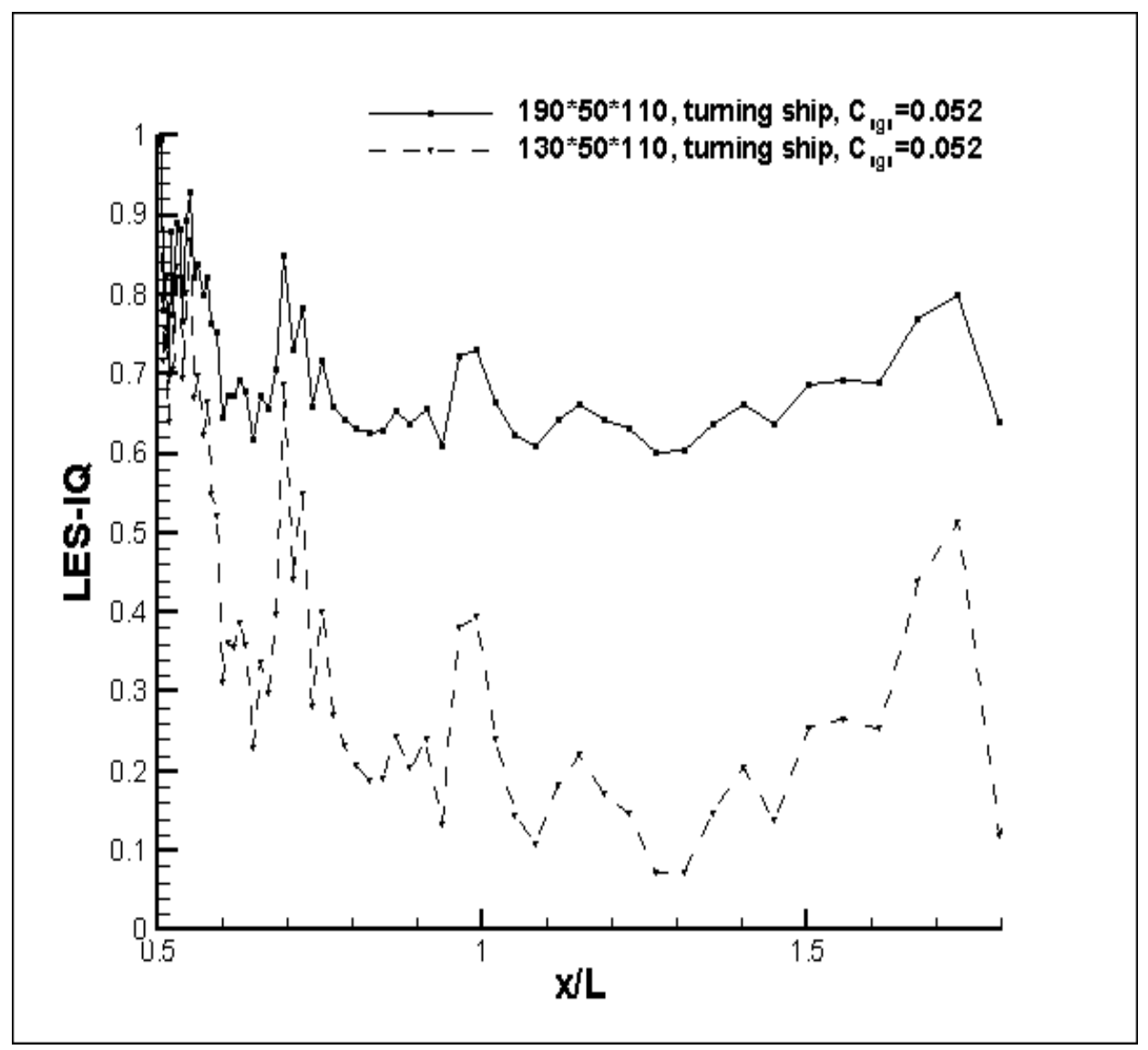

Figure 3.53. Large Eddy Simulation Index of Quality (LES_IQ) for the wake simulation of a ship cruising on a circular track 


\section{Chapter 4}

\section{CONCLUSIONS AND RECOMMENDATIONS}

\subsection{Conclusions}

This study has considered an approach to develop effective SGS models to study the turbulent flow dynamics in ship wakes. The ship hull that generated the wake has been excluded from the computational domain. To achieve this, a robust approach for providing an instantaneous velocity field in conjunction with the prescribed mean flow field, namely the random flow generation (RFG) technique (Smirnov et al., 2000, Shi et al., 2000) has been used. Reynolds Averaged Navier Stokes (RANS) calculations (Hyman, 2001) are used to provide the RFG procedure with the information needed on the inflow boundary. The further development of the wake flow is calculated via LES. A standard Smagorinsky model that forms the basis for the advanced models has been applied to assess the effects of the Coriolis and centrifugal force on the vortical structures or turbulence characteristics of the flow in the wake of a ship model (DTMB 5415) cruising on a circular track using LES technique. The eddies resolved by LES have been observed to be more energetic and less diffusive when the Coriolis force was included. It 
seems as if this force supplies energy to the large turbulent structures and thus enhances anisotropy.

Next, the physics of the wake of a ship cruising on a circular track and that of a ship cruising on a straight track have been studied in comparison to each other. The wake simulations for the ship model DTMB 5512 moving on a straight track has been compared with the macro wake measurements by Hoekstra \& Ligtelijn (1991). The prominent flow structures such as the bilge vortices are captured by LES. In measurements and simulations, the minimum axial velocity occurs near the free surface of the center of the wake. Moreover, the extent of axial turbulence intensities is in good agreement with measurements. Additional smaller side vortex pairs are observed away from the center of the wake. As the wake widens, the strength of these vortex pairs weaken. The vorticity becomes more concentrated near the free surface. Moreover, the wake spreading or wake width, which is obtained to be roughly $\mathrm{w} \sim \mathrm{x}^{1 / 4}$ is consistent with Tunaley \& Buller (1989)'s measurements. For the ship model DTMB 5415 moving on a circular track, one large vortical structure, possibly the already merged bilge vortices that moves downwards, is observed. This vortex remains at considerable strength within the distance of 1.55 ship length and then weakens. There is a smaller circulation region (a side vortex) on the outer rim of the wake. The streamwise flow field causes the side vortex to weaken and the wake decays in the outer region of the near wake similar to the ship cruising on a straight track. These large coherent structures captured by LES, which are usually smeared out by RANS simulations, are of great importance in determining the dynamics of bubbles in ship wakes. This has shown that LES is a viable tool to study complex turbulent flows, such as ship wakes. 
The classical SGS model, namely the standard Smagorinsky model, is not totally suitable for complex flows as it uses a constant eddy viscosity coefficient for the entire domain. It has been observed that the resolved eddies and the kinetic energy are sensitive to the eddy viscosity coefficient. To remedy this, some rather simple modifications are implemented on the standard Smagorinsky model (SMG). First, the SMG model has been modified to account directly for the effect of the free surface on turbulence generation. Then the behavior of various SGS models in LES of turbulent open channel flow is investigated; which include SMG without modification (W/O M), only near wall modification (NWM), and near wall modification plus free surface modification $(\mathrm{FSM}+\mathrm{NWM}$ ). When $\mathrm{FSM}+\mathrm{NWM}$ is compared to NWM and $\mathrm{W} / \mathrm{O} \mathrm{M}$, it has been observed that in near the wall region, the values are much closer to DNS of Lam and Banerjee (1992). With W/O M, the intensities in the axial flow direction increase much more compared to DNS results and other SGS models. When NWM is applied, the increase slightly diminishes and the values get closer to those obtained from DNS results. In all three models, the vertical fluctuations have decreased and its value near the free surface tends to zero as observed in experiments. However, in the free surface region $(y / \delta>0.85), F S M+N W M$ has given values that are much closer to the DNS predictions by Lam and Banerjee (1992) than other two models. The predicted turbulence intensity values have indicated similar trend and the values are in very good agreement with LES predictions of Shi et al. (2000). It has been clearly demonstrated that if there are wall boundaries, there should be wall modifications. Since the length scale should have the proper behaviour in the wall region, the original model $\mathrm{W} / \mathrm{O} \mathrm{M}$ is not recommended as a SGS model for wall-bounded flows. It has also been shown that FSM+NWM is capable 
of predicting the classical log-law profile. The results obtained have indicated that very reasonable turbulence statistics have been predicted using FSM+NWM and it can be used as a SGS model in the simulation of ship wakes.

The SMG model with FSM model has been applied to the wake behind a circular track and has been compared with the standard Smagorinsky model. The results show that with the inclusion of the free surface modification, better defined eddies are obtained and the resolved turbulent kinetic energy level on the same grid has increased. Higher frequencies and smaller turbulence scales were captured. The investigation of the effect of the free surface modeling of SGS via damping of length scales has indicated that it should be included in LES to obtain better turbulence statistics in the vicinity of free surfaces.

In this study the performance of a one equation SGS model has also been assessed. It has been demonstrated that the anisotropy of the turbulence observed near a free surface can be better simulated using a nonlinear one-equation SGS model. The whole study has given a better understanding of the role of turbulence SGS models, in predicting wall bounded free surface flows.

The validation of LES is difficult because of the fact that both the sub-grid scale (SGS) model contribution and numerical discretization errors are functions of the grid resolution. In this study, a new quality index for the assessment of the simulations claimed to be LES has been proposed as "LES_IQ". The proposed index is a measure of the percentage of the resolved turbulent kinetic energy to the total. Eq. 3.2 is recommended for those cases where $h \cong \Delta$ and the order of accuracy of the numerical scheme is approximately the same as that order implied of SGS-modeling. This method 
has been applied to the wake behind a ship cruising on a straight track and that behind a ship cruising on a circular track. It can be stated that LES_IQ is relative, such that (\%) measure means a higher $\%$ of the total turbulent kinetic energy is resolved. Further, it is up to the analyst to take this as good or bad.

\subsection{Recommendations}

Since the accuracy and efficiency are the two important points in LES of high Reynolds number flows, higher order discretization schemes must be investigated in LES code. The current solution method is only $2^{\text {nd }}$ order accurate.

The advancement of massively parallel supercomputers provides a promising way to increase the resolution of the flow by orders of magnitude. Therefore, the parallelization of the LES is strongly recommended. Sufficient number of grid resolution will certainly improve the turbulence statistics, i.e. capturing most of the energy containing eddies and the simulation will be more realistic. Adaptive grid methods may also play an important role in reducing the required number of grid points by providing high resolution only in the regions where it is necessary.

In large eddy simulations, the key for a reliable solution is to develop effective and physically correct subgrid-scale models. The concept used in free surface modifications (FSM) is promising in achieving this goal. The model has included the effects of the free surface/turbulence interaction. The application to the ship wake has given promising results. The NOEM should be tested on the ship wakes to observe the improvements in the turbulence statistics in comparison to experiments. 
Finally the present study should be extended to include the effect of surface waves so that it can be applied to high Froude number cases in the near wake region of a surface ship. Moreover, the wake flow will be more realistic with the inclusion of the movement of the free surface (wavy free surface). The free surface has been represented by a free-slip sinusoidal wall by Shi (2001) and the presence of the wave surface has been found to be significant on the distribution of the turbulent kinetic energy. 


\section{Appendix A}

\section{ENERGY SPECTRA}

The energy spectra can be used to calculate the cut-off frequency and length scales (eddy sizes) that are to be resolved by Large Eddy Simulations. The method used in this study is based on a Fourier transformation approach (Hayasa, 1999).

The energy spectrum of the U-velocity perturbation is calculated by the following formula such that

$$
\begin{aligned}
& S_{1}\left(\omega_{n}\right)=E\left[\frac{h_{T}}{M_{d}}\left|\sum_{m=1}^{M_{d}} U_{1}^{\prime}\left(t_{m}\right) \exp \left(-i \omega_{n} t_{m}\right)\right|^{2}\right]_{N_{d}} \\
& \left(n=1, \ldots . M_{d}\right)
\end{aligned}
$$

where $\omega_{n}=\frac{2 \pi n}{h_{T} M_{d}}$ is the nondimensional circular frequency, $\mathrm{M}_{\mathrm{d}}$ is the number of time series data, and $E[*]_{N_{d}}$ denotes the averaging over $\mathrm{N}_{\mathrm{d}}$ samples. Here, $\mathrm{h}_{\mathrm{T}}$ is the computational time step. 
The energy spectrum can be converted into wave number domain as,

$$
E_{1}\left(\alpha_{n}\right)=U_{c} S_{1}\left(\omega_{n}\right)
$$

where $E_{1}$ is the energy spectrum of the U-velocity perturbation in wave number domain, $\alpha=\omega_{n} / U_{c}$ is the wave number and $U_{c}$ is the mean velocity.

The algorithm and two benchmarks studied to test the algorithm has been briefly explained in Shi (2001). 


\section{Appendix B}

\section{FILTERING}

To see the effect of the filtering process that's applied through the control volume approach using significantly different length scales in different directions, let's take a two dimensional signal, $\mathrm{u}(\mathrm{x}, \mathrm{y})$ such that

$$
u(x, y)=\sum_{i=1}^{N} a_{x} \sin \left(\frac{2 \pi x}{\lambda_{x}}\right) a_{y} \cos \left(\frac{2 \pi y}{\lambda_{y}}\right)
$$

Here, $\mathrm{u}(\mathrm{x}, \mathrm{y})$ represents the Cartesian velocities in $\mathrm{x}$ - and $\mathrm{y}$-directions, $\mathrm{a}_{\mathrm{x}}$ and $\mathrm{a}_{\mathrm{y}}$ represent the amplitudes in $\mathrm{x}$ - and $\mathrm{y}$ - directions, respectively, $\lambda_{\mathrm{x}}$ and $\lambda_{\mathrm{y}}$ respresent the wave lengths in $\mathrm{x}$ - and y- directions, respectively. Figure B.1 shows the velocity color contours of the example signal, $\mathrm{u}(\mathrm{x}, \mathrm{y})$. This signal has been passed through spatial filtering of different sizes of eddies (different filter lengths; $\Delta \mathrm{x}, \Delta \mathrm{y}$ in $\mathrm{x}$ - and $\mathrm{y}$ - directions respectively) such as, 


$$
\bar{u}(x, y)=\int_{x-\frac{1}{2} \Delta x}^{x+\frac{1}{2} \Delta x} \int_{y-\frac{1}{2} \Delta y}^{y+\frac{1}{2} \Delta y} \sum_{i=1}^{N} a_{x} \sin \left(\frac{2 \pi x}{\lambda_{x}}\right) a_{y} \cos \left(\frac{2 \pi y}{\lambda_{y}}\right) d x d y
$$

Equation B.2 has been integrated numerically using the trapezoidal rule (control volume integration of Equation B.1) and the results are presented in the following figures, from Figure B.2. to B.4. In these figures, $\mathrm{u}$ represents the signal $\mathrm{u}(\mathrm{x}, \mathrm{y})$ and $\bar{u}$ is the filtered signal of $\mathrm{u}(\mathrm{x}, \mathrm{y})$. As seen from the figures, the grid size in each direction influences mostly the degree of filtering in that direction, the influence is much lesser on the other direction.

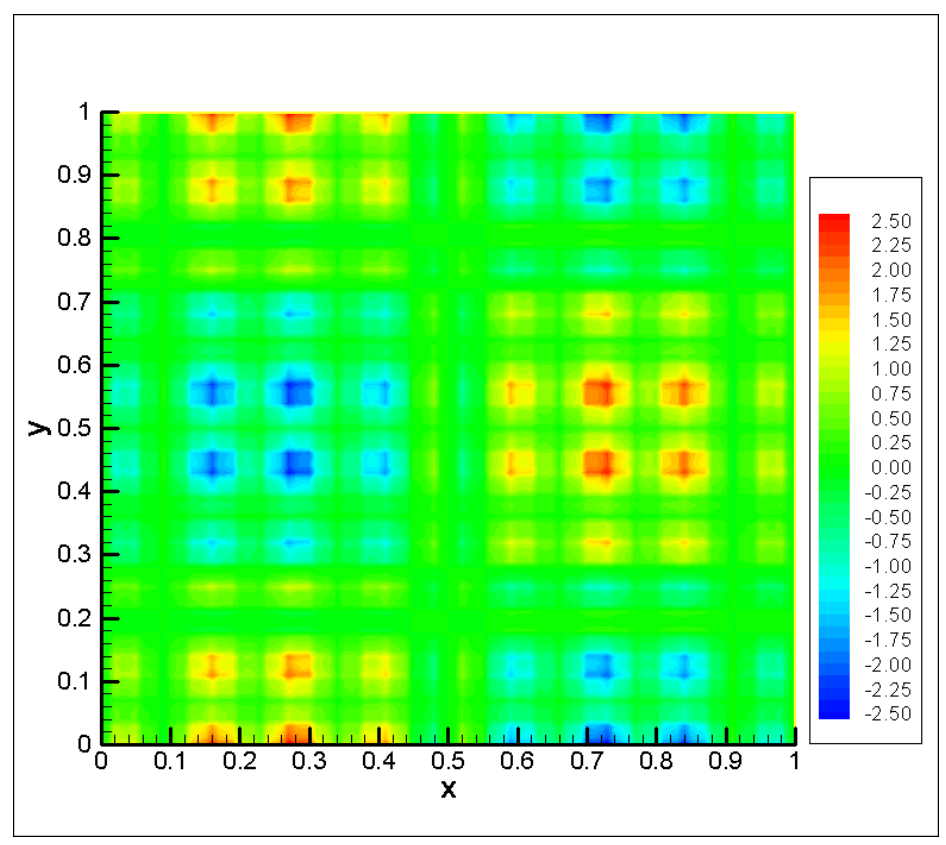

Figure B.1. Color contour of the velocity field, $u(x, y)$ 

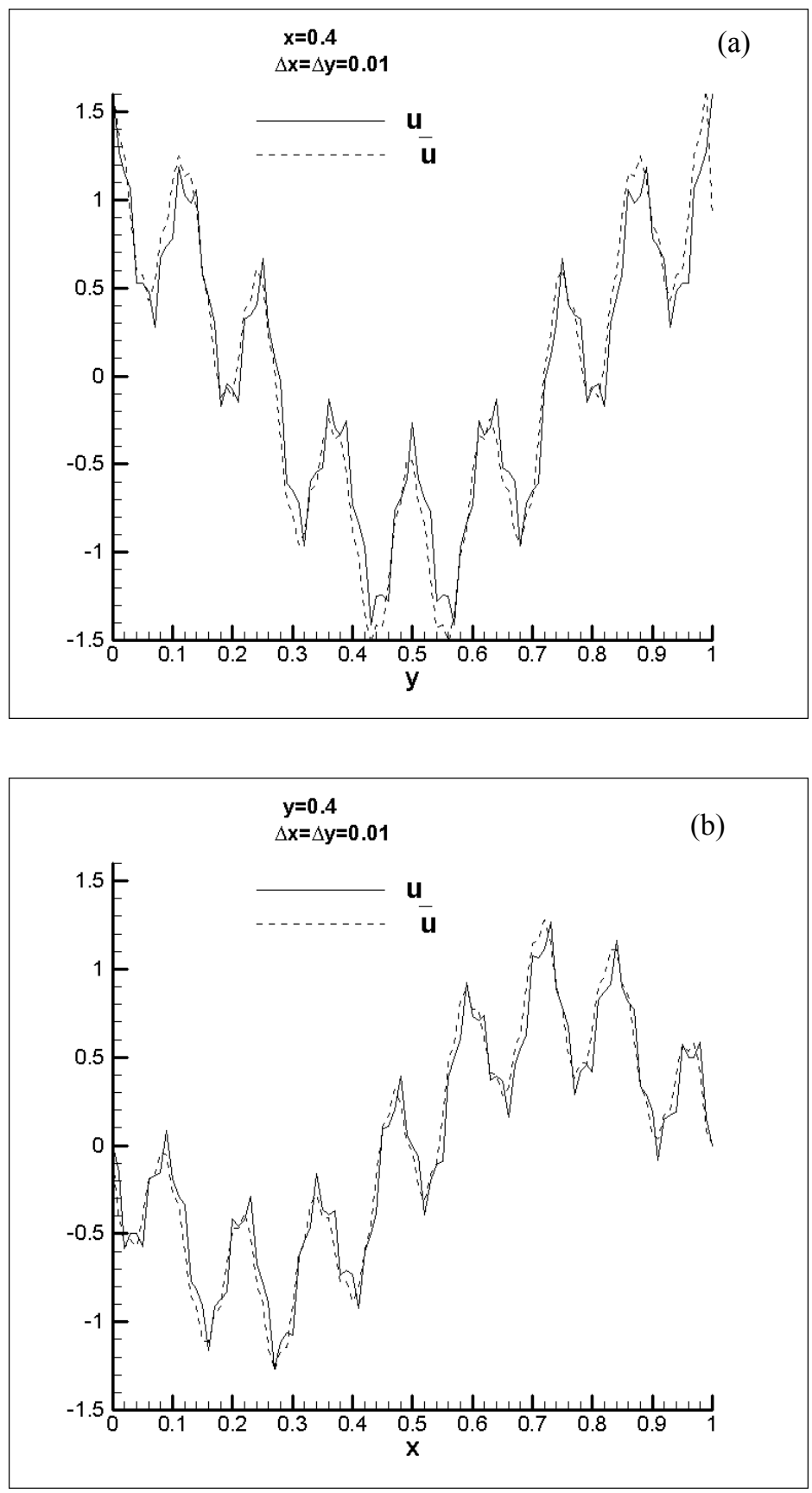

Figure B.2. a) \& b) Small filter length of equal size in both directions 

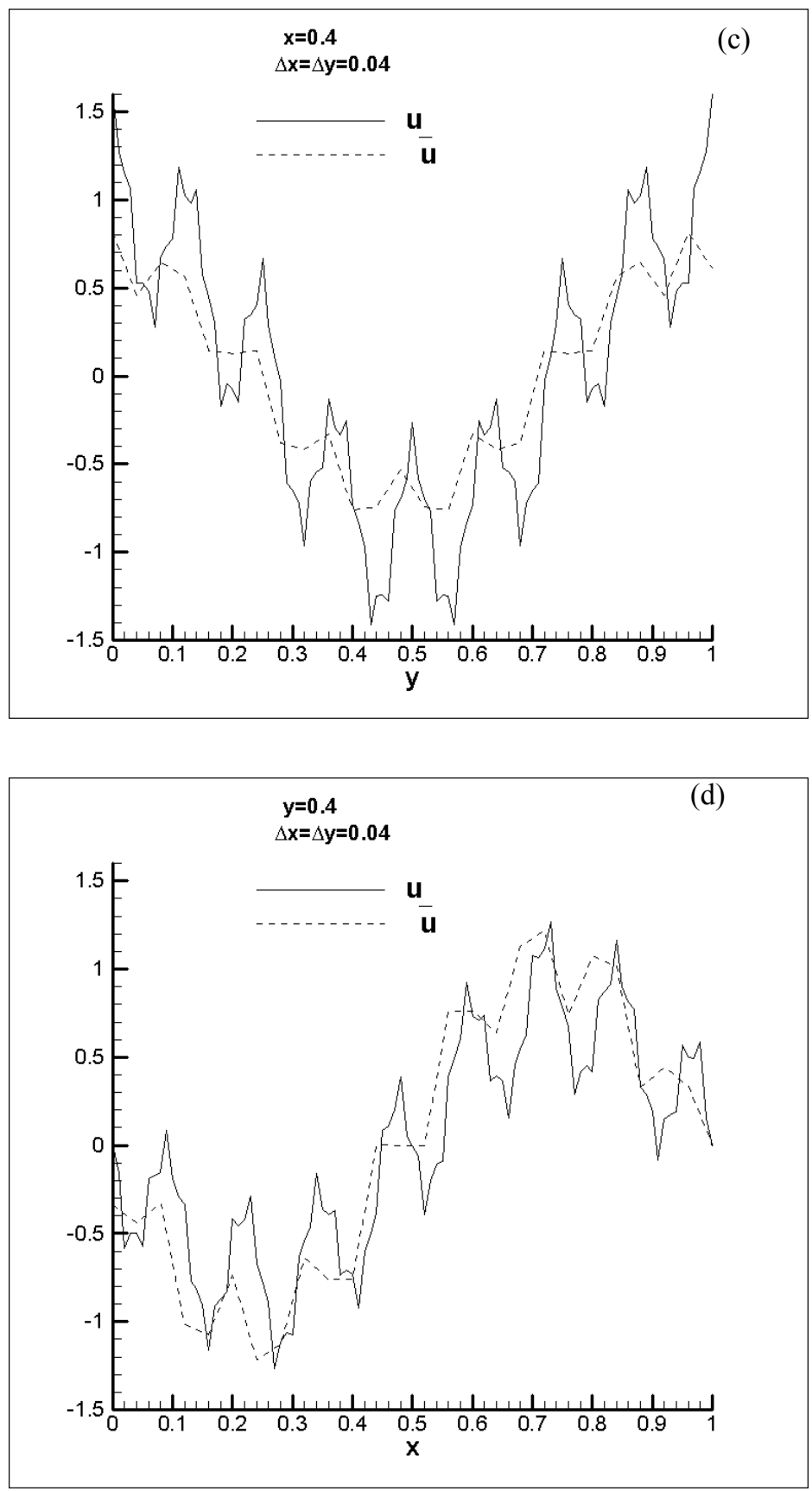

Figure B.2. c) \& d) Large filter length of equal size in both directions 

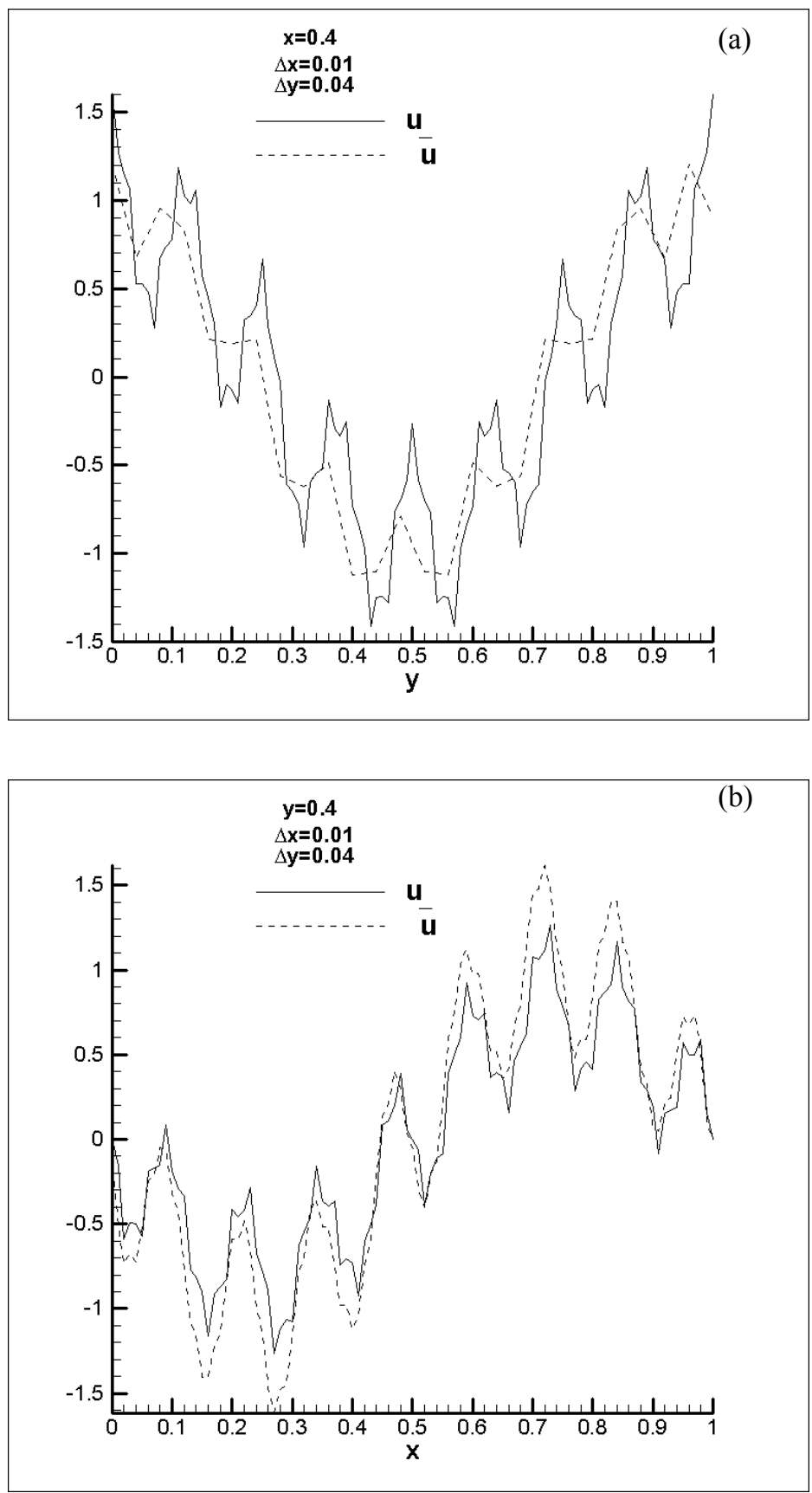

Figure B.3. a) \& b) Large filter length in the y-direction 

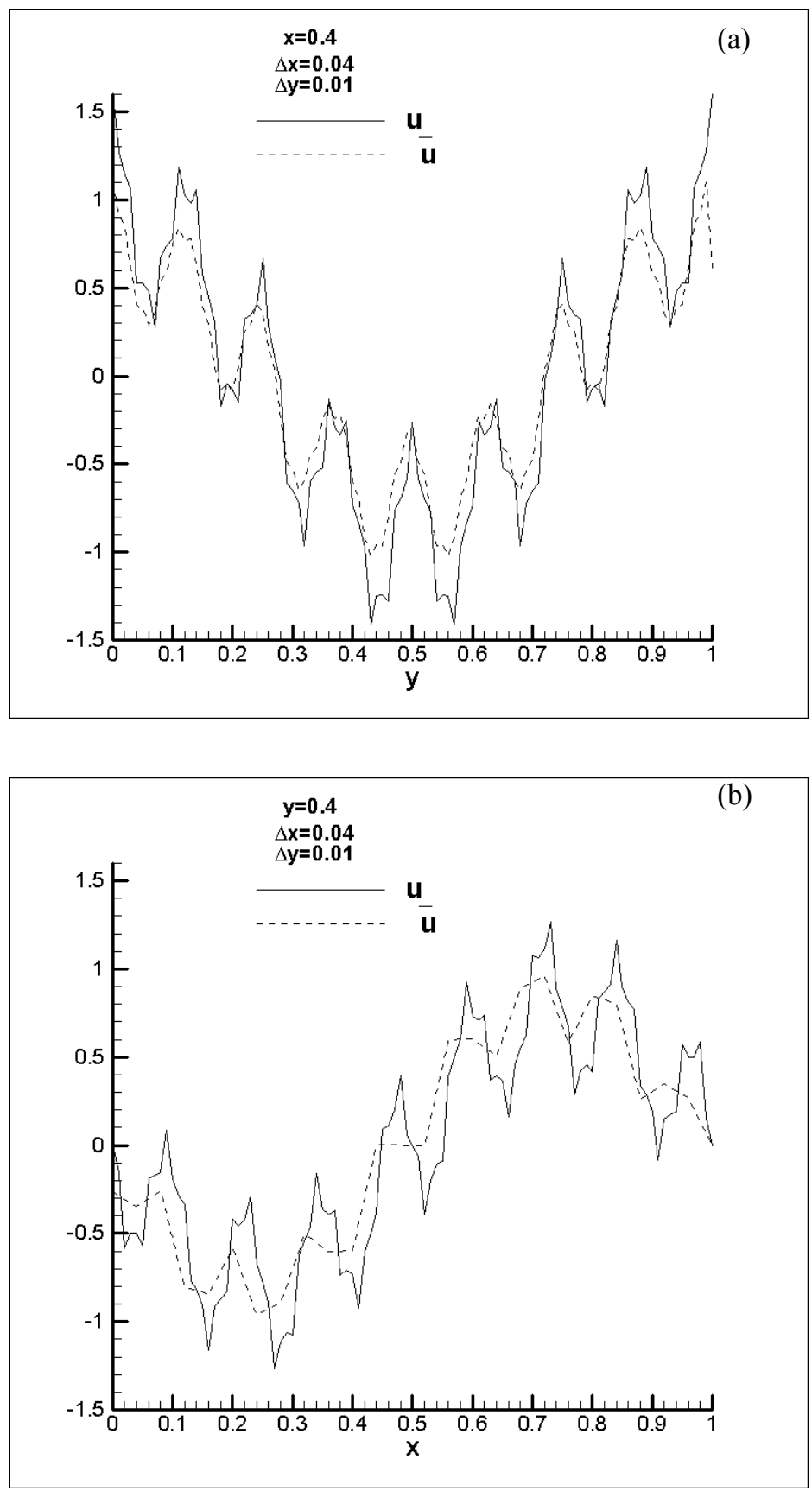

Figure B.4. a) \& b) Large filter length in the x-direction 


\section{REFERENCES}

Bardina, J, Ferziger, J.H. and Reynolds, W. C., 1980, "Improved subgrid scale models for large eddy simulation," AIAA paper, 80, 80-1357.

Benilov, A. Bang, G., Safray, A. and Tkachenko, I., 2000, "Ship Wake Detectability in the Ocean Turbulent Environment," 23 ${ }^{\text {rd }}$ Symposium of Naval Hydrodynamics, 1-15, Val de Reuil, France.

Borue, V., Orszag, S.A. and Staroselsky, I., 1995, "Interaction of surface waves with turbulence: Direct numerical simulations of turbulent open-channel flow," J.Fluid Mech. 286, 1.

Brandt, A., 1977, "Multi-level adaptive solutions to boundary-value problems," Math. Comput. 31, 333.

Celik, I., Smirnov, A., and Smith, J., 1999, “Appropriate initial and boundary conditions for LES of a ship wake," in 3rd ASME/JSME Joint Fluids Engineering Conference, Vol. FEDSM99-7851, San Francisco, California

Buller, E.H. and Tunaley, J.K.E., 1989, "The effect of the ships screws on the ship wake and its implication for the radar image of the wake," Proc. IGARSS 89, 362-365. 
Celik, I. and Rodi, W., 1984, "Simulation of Free-Surface Effects in Turbulent Channel Flows," Phys. Chem. Hydrodyn. 5, 217-227.

Celik, I., Chen, C.J., Roache, P.J. and Scheurer, G. Editors. , 1993, “Quantification of Uncertainty in Computational Fluid Dynamics," ASME Publ. No. FED-Vol. 158, ASME Fluids Engineering Division Summer Meeting, Washington, DC, 20-24 June.

Celik, I.B., Cehreli, Z.N. and Yavuz, I., 2003a, "Index of Quality for Large Eddy Simulations," Proceedings of the Forum on Turbulent Flow, 2003 ASME Fluids Engineering Division Summer Meeting, July 6-10, 2003, Hawaii.

Celik, I. B., Cehreli, Z. N. and Yavuz, I., 2003b, "Index of Resolution Quality for Large Eddy Simulations," Submitted to Journal of Fluids Engineering.

Deardoff, J.W., 1970, “A Numerical Study of Three-dimensional Turbulent Channel Flow at Large Reynolds Numbers," Journal of Fluid Mechanics 41 (2), 453-480.

Domaradzki, J.A., Liu, W. and Brachet, M.E., 1993, "An analysis of subgrid-scale interactions in numerically simulated isotropic turbulence," Phys. Fluids 5 (7), 17471759

Dommermuth, D. and Novikov, E., 1993, "Direct Numerical and Large-Eddy Simulations of Turbulent Free-Surface Flows," in Proceeding of the Sixth International Conference on Numerical Ship Hydrodynamics, 239-270, Iowa City, Iowa.

Dommermuth, D., Gharib, M., Huang, H., Innis, G., Maheo, P., Novikov , E., Talcott, J., and Wyatt, D., 1996, "Turbulent Free-Surface Flows: A Comparison Between Numerical Simulations and Experimental Measurements," $21^{\text {st }}$ Symposium of Naval Hydrodynamics, 200-215, Trondheim, Norway. 
Durbin, P.A., 1995, "Separated flow computations with the k-e-v² model," AIAA J 33 659-664.

Farmer, J., Martinelli, L., Jameson, A., 1994, "Fast Multigrid method for solving incompressible hydrodynamic problems with free surfaces," AIAAJ. 32, 1175.

Ferziger, J., 1993, Subgrid Scale Modeling, Large Eddy Simulation of Complex Engineering and Geophysical Flows, edited by Galperin, B. and Orszag, S., Cambridge University Press.

Ferziger, J. and Peric, M., 1997, Computational Methods for Fluid Dynamics, Springer, Mercedesdruck, Berlin.

Freitas, C.J., 1993, “Journal of Fluids Engineering Editorial Policy Statement on the Control of Numerical Accuracy," Journal of Fluids Engineering, Vol. 115, pp. 339340.

Germano, M., Piomelli, U., Moin, P. and Cabot, W.H., 1991, “A dynamic subgrid scale eddy viscosity model," Phys. Fluids A 3, 1760-1765.

Ghosal, S., Lund, T., Moin, P., and Akselvoll, K., 1995, “A dynamic localization model for large-eddy simulation of turbulent flows," J. Fluid Mech. 286,229.

Gonzalez, J.A., Melching, C.S. and Oberg, K.A., 1996, “Analysis of Open-Channel Velocity Measurements Collected with the Acoustic Doppler Current Profiler," Proc., $1^{\text {st }}$ International Conference On New/Emerging Concepts for Rivers, Chicago, Illinois.

Gui, L., Longo, L., and Stern, F., 1999, “Towing Tank PIV Measurements System and Data and Uncertainty Assessment for DTMB Model 5512," $3^{\text {rd }}$ International Workshop on PIV, Santa Barbara, CA, 16-18 September. 
Hamba, F., 2001, “An Attempt to Combine Large Eddy Simulation with the k- $\varepsilon$ Model in a Channel-Flow Calculation,” Theoret. Comput. Fluid Dynamics 14, 323-336.

Handler, R. A., Swean, T. F., Leighton, R. I. and Swearingen, J. D., 1993, "Length scales and the energy balance for turbulence near a free surface," AIAA 31, 1998-2007.

Hayasa, T., 1999, "Monotonic convergence property of turbulent flow solution with central difference and quick schemes," Journal of Fluids Engineering 121, 351.

Hennings, I., Alpers, W. and Romeiser, R., 1999, "Radar Imaging of Kelvin arms of ship wakes,” Int. J. Remote Sens., 20 (13), 2519-2543.

Hoekstra, M. and Ligtelijn, J., 1991, "Macro Wake Features of a Range of Ships," MARIN Report No. 410461-1-PV, Netherlands.

Hoekstra, M. and Aalbers, A., 1997, "Macro Wake Measurements for a Range of Ships," $21^{\text {st }}$ Symposium of Naval Hydrodynamics, 278-290, Trondheim, Norway.

Hunt, J.C.R, 1984, Turbulence structure and turbulent diffusion near gas-liquid interfaces, Gas Transfer at Water Surfaces (eds. W.Brutsaert \& G.H. Jirka), Reidel Pub., 67-82.

Hyman, M., 1998, "Computation of ship wake flows with free-surface/turbulence interaction," in $22^{\text {nd }}$ Symposium on Naval Hydrodynamics, 11-32, Washington, D.C.

Hyman, M., 2001, Private Communications.

Jordan, S., 1999, “A large-eddy simulation methodology in generalized curvilinear coordinates," Journal of Computational Physics 148, 322.

Kalitzin, G., 1999, “Application of the $\mathrm{v}^{2}-\mathrm{f}$ model to aerospace configurations," Center for Turbulence Research Annual Research Briefs, 289-300. 
Kim, J. and Moin, P., 1985, "Application of a fractional step method to incompressible Navier-Stokes equations," Journal of Computational Physics 59, 308.

Krajnovic, S. and Davidson, L., 1999, "Large Eddy simulation of the flow around a surface mounted cube using a dynamic one equation subgrid model," TSFP-1, 741746, Eds: S. Banerjee \& J.K. Eaton, begell house, inc.

Komori, S., Ueda, H., Ogino, F. and Mizushina, T., 1982, "Turbulence structure and transport mechanism at the free surface in an open channel flow," Inter. J. of Heat and Mass Transfer, 25 (4), 513-521.

Komori, S., Hiraga, Y., Murakami, Y. and H. Ueda, 1987, "The generation of surfacerenewal eddies in an open channel flow," Proc. of $2^{\text {nd }}$ Int. Sym. on Transport Phenomena in Turbulent Flows, Tokyo, 75-85.

Komori, S., Nagaosa, R. and Y. Murakami, 1990, "Mass transfer into a turbulent liquid across the zero-shear gas-liquid interface," AIChE 36, 957.

Komori, S., Nagaosa, R., Murakami, Y., Chiba, S., Ishii, K. and Kuwahara, K., 1993, "Direct numerical simulation of three dimensional open channel flow with zero-shear gas-liquid interface," Phys. Fluids A 5 (1), 115-125.

Kraichnan, R., 1970, “Diffusion by a Random Velocity Field,” Phys. Fluids 11, 43.

Lam, K. and S. Banerjee, 1992, "On the condition of streak formation in a bounded turbulent flow," Phys. Fluids A 4, 306.

Leonard, A. 1974, "Energy cascade in large eddy simulations of turbulent fluid flows," Adv. Geophys., 18A, 237-248.

Leonard, B., 1988, "Simple high accuracy resolution program for convective modeling of discontinuities," International Journal for Numerical Methods in Fluids 8, 1291. 
Lewellen, W., 1977, "Use of invariant modeling," in Handbook of turbulence, Plenum. Lilly, D., 1967, "The representation of small scale turbulence in numerical experiments," in Proc. IBM Scientific Computing Symposium on Environmental Science, White Plains, NY.

Lilly, D.K., 1992, "A proposed modification of the Germano subgrid scale closure method," Phys. Fluids A 4, 633-635.

Lyn, D., Einav, S., Rodi, W. and Park, J., 1995, "A laser-doppler velocimetry study of ensemble-averaged characteristics of the turbulent near wake of a square cylinder," Journal of Fluids Mechanics 304, 285.

Lumley J.L., 1978, "Computational modelling of turbulent flows," Advances in Applied Mechanics 18, 123-176.

Longo, J., Stern, F., and Toda, Y., 1993, "Mean-Flow Measurements in the Boundary Layer and Wake and Wave Field of a Series $60 \mathrm{CB}=0.6$ Ship Model - Part 2: Scale Effects on Near-Field Wave Patterns and Comparisons with Inviscid Theory," J. Ship Research, 37 (1), 16-24.

Madnia, K., 1989, Interaction of a Round Jet with a Free Surface, Ph. D. Thesis, University of Michigan.

Mason, P.J. and Thomson, D.J., 1992, "Stochastic backscatter in large-eddy simulations of boundary layers," J. Fluid Mech. 242, 51-78.

Matsubara, M. and Alfredsson, P.H., 1998, "Secondary instability in rotating channel flow," J. Fluid Mech. 368, 27-50. 
McMillan, O. J., Ferziger, J. H. and Rogallo, R. S., 1980, “Tests of subgrid-scale models in strained turbulence," AIAA Fluid and Plasma Dynamics Conference, 13th, Snowmass, Colo., July 14-16, 1980, 11.

Meadows, L., Meadows, G., Troesch, A., Cohen, S., Beier, K.-P., Root, G., Griffin, O. M., Swean, T.F., Jr., 1994, "Lagrangian Velocity Profiles in the Wake of a High Speed Vessel," Ocean Engineering, 21, (2), 221-242.

Melsheimer, C., Lim, H. and Shen, C., 1999, “Observation and Analysis of Ship Wakes in ERS-SAR and SPOT Images," Proc. 20th. Asian Conference for Remote Sensing, 22-25 November, 1999, Hong Kong, China.

Menon, S. and Yeung, P.-K., 1995, “Analysis of Subgrid Models using Direct and LargeEddy Simulations of Isotropic Turbulent Flows," Proceedings of the AGARD Meeting on "Application of Large-Eddy Simulations in Transition and Turbulent Flows", AGARD CP-551, 10.1-10.12

Menon, S. and Kim, W.-W., 1996, "High Reynolds Number Flow Simulations using the Localized Dynamic Subgrid Scale Model," 34th Aerospace Sciences Meeting, AIAA Paper No. 96-0425.

Milgram, J.H., 1988, “Theory of Radar Backscatter from Short Waves Generated by Ships, with Application to Radar (SAR) Imagery,”J. Ship Res,. 32 (1), 54-69.

Milgram, J.H., Peltzer, R.D. and Griffin, O. M., 1993, "Suppression of Short Sea Waves in Ship Wakes: Measurements and Observations," J. Geophysics Res. 98 (C4), 71037114.

Moin, P. and Kim, J., 1982, "Numerical investigation of turbulent channel flow," J. Fluid Mech 118, 341 . 
Nakayama, A. and Yokojima, S., 2002, "LES of Open-Channel Flow with Free-Surface Fluctuation," Annual Journal of Hydraulic Eng., JSCE 46.

Naudascher, E., 1965, "Flow in the wake of Self-Propelled Bodies and Related Sources of Turbulence," J. Fluid Mech. 22 (1), 625-656.

Nezu, I., 1977, Turbulent structure in open channel flows. Ph D Thesis presented to Kyoto University, Kyoto, Japan.

Nezu, I. and Rodi, W., 1985, "Experimental study on secondary currents in open channel flows," Proc. $21^{\text {st }}$ Congress of IAHR 2, Melbourne, Australia, 115-119.

Nezu, I. and Nakagawa, H., 1986, "Investigation on three-dimensional turbulent structure in uniform open-channel and closed ducts," Proc. Japan Soc. Civil Engrs. 369, 89-98.

Nezu, I. and Rodi, W., 1986, "Open channel flow measurements with a laser Doppler anemometer," J. Hydraulic Eng., ASCE 112, 335-355.

Nezu, I. and Nakagawa, H., 1993, Turbulence in Open Channel Flows, Balkema, Rotterdam.

Nisizima, S. and Yoshizawa, A., 1987, "Turbulent channel and couette flows using an anisotropic k-e model," American Institute of Aeronautics Journal 25 (3), 414-420.

Patankar, S.V., 1980, Numerical Heat Transfer and Fluid Flow, Hemisphere Publishing Corporation, New York, N.Y.

Paterson, E., Hyman, M., Stern, F., Bonetto, F., Drew, D. and Lahey, R., 1996, "Nearand far-field CFD for a Naval Combatant including thermal-stratification and twofluid modeling," $21^{\text {st }}$ Symposium on Naval Hydrodynamics, 102-117.

Paterson, E., 2003, Private Communications. 
Piomelli, U., 1993, "High Reynolds number calculations using dynamic subgrid scale stress model," Phys. Fluids A 5, 1484-1490.

Piomelli, U., Coleman, G. N. and Kim, J., 1997, "On the effects of nonequlibrium on the sub-grid scale stresses," Phys. Fluids 9 (9), 2740-2748.

Piomelli, U., 1998, "Large Eddy Simulations: Present State and Future Directions, ” AIAA Paper 98-0534.

Piomelli, U., 1999, "Large Eddy simulation: achievements and challenges," Prog. Aerosp. Sci. 35, 335-362.

Rai, M. and Moin, P., 1991, "Direct simulation of turbulent flows using finite-difference schemes," Journal of computational physics 109 (2), 169.

Ratcliffe, T., 1998, "Validation of free surface Reynolds averaged Navier Stokes and Potential flow codes," $22^{\text {nd }}$ Symposium on Naval Hydrodynamics, 964-980.

Reed, A. M., Beck, R. F, Griffin, O. M. and Peltzer, R. D., 1990, "Hydrodynamics of Remotely Sensed Surface Ship Wakes," SNAME Transactions 98, 319-363.

Rhie, C.M. and Chow, W.L., 1983, "Numerical study of the turbulent flow past an airfoil with trailing edge separation," AIAA 21 (11), 1525-1532.

Rightley, P., 1995, Bubble dispersion and interphase coupling in a free shear flow, Ph.D. Thesis, University of California, San Diego, CA.

Roache, P. J., Ghia, K. N. and White, F. M., 1986, "Editorial Policy Statement on Control of Numerical Accuracy," ASME Journal of Fluids Engineering 108, Mach Issue.

Roache, P. J., 1998, Verification and Validation in Computational Science and Engineering, Hermosa Publishers, Albuquerque. 
Rood, E. P., 1998, "Analytic Investigation of the Increase in Kinetic Energy at the ShearFree Constant-Pressure Boundary of a Fluctuating Flow," JSME Fluids and Thermal Engineering 41 (1).

Rubinstein, R. and Barton, J. M., 1990, "Nonlinear Reynolds stress models and the renormalization group,"Physics of Fluids A 2 (8), 1472-1476.

Salvetti, M. V., Zang, Y., Street, R. L., Banerjee, S., 1997, "Large-eddy simulation of free- surface decaying turbulence with dynamic subgrid-scale models," Phys. Fluids 9 (8).

Sarpkaya, T., Magee, M. and Merrill, C., 1994, "Vortices, free surface and turbulence," FED (181), Free surface Turbulence, 1-14, ASME.

Smagorinsky, J., 1963, "General circulation experiments with the primitive equations; I. The basic equations," Mon. Weather Rev 91, 99-164.

Smirnov, A., Shi, S. and Celik, I., 2000, "Random Flow simulations with a bubble dynamics model," ASME Fluids Engineering Division Summer Meeting 11215, FEDSM2000, Boston, MA.

Smirnov, A., Shi, S. and Celik, I., 2001, "Random Flow Generation Technique for Large Eddy Simulations and Particle Dynamics Modeling" JFE 123.

Shi, J., Thomas, T. G., Williams, J. J. R., 2000, "Free-surface Effects in Open Channel Flow at Moderate Froude and Reynolds Numbers," Journal of Hydraulic Research 38 (6), 465-474.

Shi, S., Smirnov, A. and Celik, I., 2000, "Large Eddy Simulations of turbulent wake flows," Twenty-Third Symposium on Naval Hydrodynamics, 203-209, Val de Reuil, France. 
Shi, S., 2001, Towards Large Eddy Simulation of Ship Wakes, Ph.D. Thesis, West Virginia University.

Shi , S., Celik, I. and Smirnov, A., 2002, "LES of a Spatially Developing Turbulent Wake Flows," Journal of Ship Research.

Smutek, R., 1969, Discussion on 'Measurement of turbulence in water' by Richardson and McQuivey, J.Hydraulics Div., ASCE 95 (HY-1), 519-523.

Sohankar, A., Davidson, L., and Norberg, C., 2000, "Large Eddy Simulation of Flow Past a Square Cylinder: Comparison of different subgrid scale models," Journal of Fluids Engineering 122, 39-47.

Sohankar A, Norberg C and Davidson L., 1999, "Simulation of three-dimensional Flow around a square cylinder at moderate Reynolds numbers," Physics of Fluids 11, 288306.

Speziale, C.G., 1987, “On nonlinear k-1 and k-e models of turbulence,” Journal of Fluid Mechanics 178, 459-475.

Speziale, C. G., 1998, “Turbulence Modeling for Time-Dependent RANS and VLES: A Review," AIAA 36 (2).

Stern, F., Longo, J., Penna, R., Olivieri, A., Ratcliffe, T., and Coleman, H., 2000a, "International Collaboration on Benchmark CFD Validation data for Surface Combatant DTMB Model 5415”, 23 ${ }^{\text {rd }}$ Symp. On Naval Hydrodynamics, 17-22 Sept 2000, Val de Reuil, France.

Stern, F. and Wilson, R., 2000b, "RANS solution of Ship Wake," University of IOWA, Private communication. 
Stern, F., Wilson, R. V., Coleman, H. W. and Paterson, E. G., 2001, "Comprehensive Approach to Verification and Validation of CFD Simulations - Part 1: Methodology and Procedures," ASME Journal of Fluids Engineering 123, pp. 793-802.

Swean, T.F., Jr., 1987, "Numerical Simulations of the Wake Downstream of a TwinScrew Destroyer Model,” Naval Research Laboratory Memorandum Report 6131, 41 p.

Thompson, W. (Lord Kelvin), 1887, “On Ship Waves”, Proc. Inst. Mech. Eng., [Popular lectures, iii.482]; Lamb, H., "Hydrodynamics”, (Dover, New York, 1985), 434.

Ueda, H., Moller, R., Komori, S. and Mizushina, T., 1977, "Eddy diffusivity near the free surface of open channel flow," Int. J. Heat and Mass Transfer 20, 1127-1136.

Vreman, B., Geurts, B. and Kuerten, H., 1997, "Large-eddy simulation of the turbulent mixing layer," J.Fluid Mech. 339, 357-390.

Wilcox, D.C., 1993, Turbulence modeling for CFD, La Canada, CA: DCW Industries.

Yavuz, I., Cehreli, Z. N. and Celik, I. B., 2002, "Large Eddy Simulation of the Wake Behind a Turning Ship", 2002 Fluids Engineering Division Summer Meeting, July 14-18, 2002, Montreal, Quebec, Canada.

Yokojima, S. and Nakayama, A., 2001, "DNS study of Froude number effects on turbulence structure near free surface," J.Applied Mech. JSCE 4, 619-629.

Yoshizawa, A. and Horiuti, K., 1985, "A statistically-derived-subgrid-scale kinetic energy model for the large eddy simulation of turbulent flows," Journal of the Physical Society of Japan 54 (8), 2834.

Zang, Y., 1993, On the development of tools for the simulation of geophysical flows, Ph.D. Thesis, Stanford University. 
Zang, Y., Street, R. L. and Koseff, J. R., 1993, “A dynamic mixed subgrid-scale model and its application to turbulent recirculating flows," Phys. Fluids 5 (12), 3186.

Zang, Y., Street, R. L. and Koseff, J. R., 1994, “A Non-staggered Grid, Fractional Step Method for Time-Dependent Incompressible Navier-Stokes Equations in Curvilinear Coordinates," J. Comp. Phys. 114, 18-33. 